\title{
ON THE BALANCE OF FORCES AND \\ RADIAL ACCELERATIONS IN HURRICANES
}

by

William M. Gray

A Report on Research Conducted Under Contracts

Between The United States Weather Bureau, Colorado State University and

The University of Chicago.

February 1961

Atmospheric Sciences Research Report Number 1. 
TABLE OF CONTENTS

Page

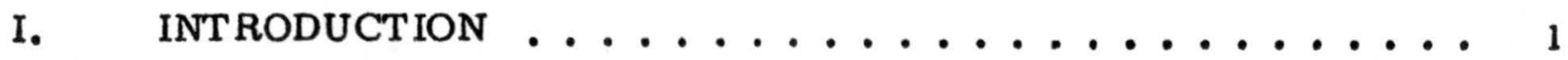

II. EQUATIONS OF MOTION AND COMPUTATIONAL PROCEDURE. 5

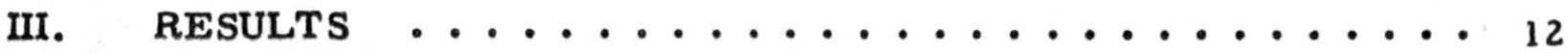

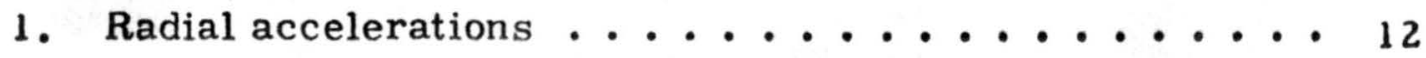

a. relative to the fixed storm center $\left.-\dot{\mathrm{v}}_{\mathbf{r}}\right)_{\mathrm{gr}} \ldots 12$

b. relative to the moving storm center - $\dot{v}_{\mathbf{r r}}^{\prime} \mathbf{g r}^{\prime} 13$

2. Relation of $\dot{v}_{r_{g r}}$ to $v_{r}$ and the maximum wind $\ldots . .16$

3. Comparison of Asymmetries of $g \partial D / \partial r$ and $\left(\overline{f v}_{\theta}+\overline{\mathrm{v}}_{\theta}^{2} / \mathrm{r}\right)$ around the storm $\ldots \ldots . . . . .17$

4. Further Analysis of the $\dot{\mathrm{v}}_{\mathrm{r}}$ ' gr $^{\text {Field in Stationary }}$

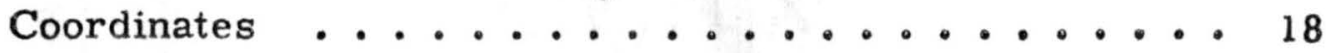

5. Preliminary Comments on the Magnitude of $\mathrm{F}_{\mathrm{r}} \ldots 21$

6. Radius of Trajectory Curvature .......... 22

7. Condensation Heating ............ 25

a. related to radial friction and deppening tendency . 25

b. related to the wind and pressure field in

Hurricane Daisy on 25 August ........ 27

IV. SUMMARY OF PRINCIPAL RESULTS .......... 30

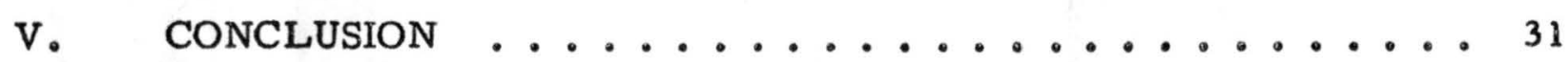

vi ACKNOWLEDGEMENTS ................... 33

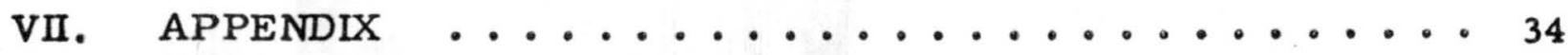

1. Reliability of the Computations ......... 35

2. Equality of Frictional Acceleration in Fixed and

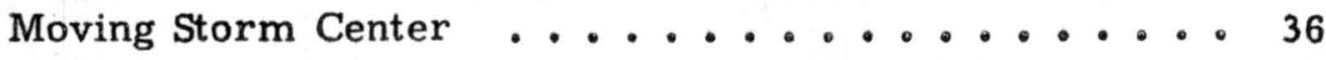

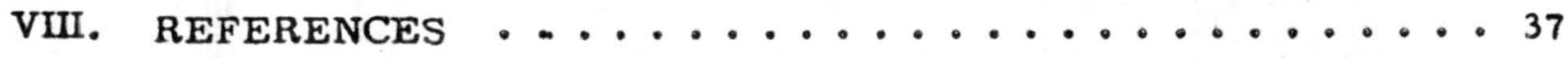

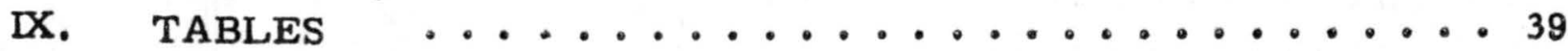

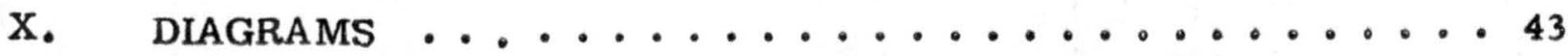




\section{INTRODUCTION}

Hurricanes have often been described as vortices in which the cyclostrophic wind equation should be valid in the core of maximum winds and the gradient wind equation in the periphery. But the degree to which these equations really describe the balance or forces has not been determined. This has been due to lack of reliable wind and pressure-height information. With the installation of the National Hurricane Research Project of the United States Weather Bureau in 1956, and with technical development of the gyronavigation system and other instruments, these observational inadequacies have been reduced and to some extent overcome. The Doppler navigation system has given for the first time accurate wind data over ocean areas. Within the limits of accuracy of observations, the question concerning the form of the laws of motion applicable in hurricanes can now be answered in certain respects for several storms which were thoroughly covered by research missions of the National Hurricane Research Project.

This paper presents a first attempt to take up some special aspects of the balance of forces computed from the flight data. Computations are made for Atlantic hurricanes Carrie (1957), Cleo (1958), Daisy (1958), and Helene (1958).

Instrumentation of Aircraft. -- During the hurricane seasons of 19571958, the National Hurricane Research Project operated three aircraft in conjunction with the U.S. Air Force. There were two B-50's and one B-47. The instrumentation installed and maintained in these planes has been described by Hilleary and Christensen (4). It will therefore suffice to note that the instrumentation of interest for this study consisted of a Doppler (gyro) Navigation wind measurement, radio and pressure altimeter for determination of the altimeter correction (" $D ")$, and a vortex thermometer for temperature measurements. Punch card systems were installed in the back of the aircraft. 
Instantaneous values of the above parameters were punched at specified time intervals-- one card every two to ten seconds--with the more rapid punching in the hurricane cores. The punch card decks were taken to National Hurricane Research Project after each mission and evaluated by machine processing. Note was taken of the hurricane displacement over the duration of the mission and all quantities were plotted in a latitude-longitude coordinate system fixed with respect to the storm center.

In this study, use is made of the horizontal winds and the observed " $D$ " values. Temperatures are used only for checking on the computed vertical thicknesses, obtained from the " $D$ " values between isobaric surfaces.

Research Missions in Hurricane Daisy (1958). --Hurricane Daisy began to form on August 24, just to the northeast of the Bahama Islands (Fig. 1). By 1200 GMT on August 25th, the maximum winds in the core were 70 knots. It was at this time that National Hurricane Research Project aircraft first entered Daisy. One Air Force B-50 made radial penetrations into the eye at $830 \mathrm{mb}$; additional legs were executed at 1600 and 2800 feet. The second B-50 operated in the middle troposphere at $570 \mathrm{mb}$. The B-47 aircraft carried out a mission in and around the storm at $237 \mathrm{mb}$.

Two missions were flown into Daisy on the 26th, but without radial penetration. On August 27th and 28th, radial penetrations were made at 630 and $620 \mathrm{mb}$ respectively. Daisy reached its peak intensity on the 27 th, with maximum winds above 115 knots. A plot of central pressure with time is given in Fig. 2 .

Mission into Hurricane Cleo (1958). -- Cleo formed in an easterly wave west of the Canary Islands on August 9th. It moved steadily westward for six days to approximately $15^{\circ} \mathrm{N}, 48^{\circ} \mathrm{W}$, and then turned northward moving steadily in this direction until it took on extra-tropical characteristics SE of Newfoundland on the 19th. It reached maximum intensity on the 14th, just before turning northward. The maximum winds were approximately 120 knots 
and minimum pressure $945 \mathrm{mb}$. A three plane National Hurricane Research Project mission was flown into the storm on the 18th of August when Clea was moving in an approximately steady state toward the NNE at 14 knots. Maximum winds were approximately 95 knots and minimum pressure $970 \mathrm{mb}$. The storm eye was nearly 25 miles in diameter and the eye wall clouds quite extensive. One mission was accomplished at $820 \mathrm{mb}$, another at $580 \mathrm{mb}$, and a third at $255 \mathrm{mb}$. See storm track Fig. 3 .

Missions into Hurricane Carrie (1957). -- Carrie formed from an easterly wave near $20^{\circ} \mathrm{N}, 35^{\circ} \mathrm{W}$, on September 5 . It moved steadily west and northwest. By September 15 it was located at $30^{\circ} \mathrm{N}, 58^{\circ} \mathrm{W}$, moving to the NW at 12 knots in approximate steady-state. Its central pressure was $965 \mathrm{mb}$ and maximum winds 85 knots. A mid-tropospheric probe $(605 \mathrm{mb})$ was made at this time. Another mid-tropospheric flight into Carrie $(690 \mathrm{mb})$ was made on the 17th of September when the storm had changed direction and was moving toward the ENE at 13 knots. Minimum pressure and maximum winds were nearly the same as on the 15th. See the storm track Fig. 4 .

Missions in Hurricane Helene (1958). -- This storm started to develop on September 22 at $22^{\circ} \mathrm{N}, 65^{\circ} \mathrm{W}$, or $200 \mathrm{n}$. mi. NNE of San Juan, P.R. It moved almost directly northwestward for the next four days, while slowly intensifying (see storm track Fig. 5). A flight at $635 \mathrm{mb}$ was made on September 24 when the central pressure was approximately $995 \mathrm{mb}$ and maximum winds were 60 knots. Another flight was made on September 25 at $810 \mathrm{mb}$ when the storms central pressure was approximately $980 \mathrm{mb}$ and maximum winds approximately 90 knots. Other flights into Helene were made by National Hurricane Research Project on the 26th of September. Only the results of the two flights on the 24th and 25th are presented in this paper.

Each day's data was collected in six to ten hours between the approximate hours of 1400 to 2400 GMT. For computational purposes, an essentially steady state must be assumed for this period of flight, even though some changes could be noted when aircraft repeatedly passed the same point with respect to the storm center. 
Table I contains the storms, levels, dates, maximum winds, etc. to which computations were performed. The ten levels with the asterisk are those where at least six radial legs were flown (except Cleo $820 \mathrm{mb}$ which had five radial legs). It is at these levels where field analysis of computed values could be made. A typical sample of a flight path along one of these levels is shown in Fig. 10. 
Aircraft observations of necessity are taken in a mixed space-time coordinate system. But, as already stated, usually the complexity of the reference frame can be reduced by the assumption, usually valid in the first approximation, that the storm remains in essentially steady state for the duration of a flight mission. Granted this premise, all observations can be assigned a location in a coordinate system fixed with respect to the moving center. Given a plot of the data in this form, an investigation of the forces and accelerations present may be provided from a variety of viewpoints. Three of the most common approaches are the following:

1) Computation of the actual forces with respect to the instantaneous center position along the radial and tangential axis of a polar coordinate system.

2) A similar computation in a moving reference frame where at first the translatory storm velocity is subtracted from all winds, so that the wind field studied is that relative to the moving center.

3) Computations in a natural coordinate system in which the forces acting on individual air particles are determined.

The last system, a Lagrangian one, is in some respects the most attractive. But computations depend on knowledge of the radius of trajectory curvature, a quantity which is not measured but must be obtained by indirect means based on the field distribution of data. In such a procedure the shortcomings of the steady state assumption and of the frequent wide gaps between flight legs are essential.

Now in hurricanes, much interest reasonably centers on the radial accelerations since they are related to the mass flow through the system and have the energy releases which produce and maintain these cyclones. Given the objective of investigating these accelerations, the first two coordinate systems are not subject to the difficulties encountered in the natural frame, 
so all requisite data are furnished by single radial flight legs of perhaps 15 minutes duration and the whole job can be done by machine process if desired. In contrast, calculations along the tangential direction not only give less promise of insight into the hurricane but they are also difficult to accomplish since airplanes cannot fly circles around hurricanes and since gradients of wind and pressure usually are very weak along this axis compared to the radial axis.

Since the course to be pursued will be to determine whatever is possible about radial accelerations, most calculations will be made with respect to the disturbances center position since it is felt that the greatest importance resides in the radial coordinates. Later, however, the transformation to moving coordinates will be made as there is the advantage that local time changes are eliminated, and advective changes are then referred to the storm's center rather than to a point fixed on earth. Finally, radii of trajectory curvature will be determined from the polar coordinate grid at certain special points. This calculation will be made and the results compared with approximations of the radius of trajectory curvature from kinematic methods.

The polar coordinate system will be applied to constant pressure surfaces which then are treated as quasi-horizontal surfaces, where it may be noted that even in the areas with strongest pressure gradient the slope of a constant pressure surface will not exceed the order of 1:100 (pressure gradient of $2 \mathrm{mb} / \mathrm{mile})$. The radius $r$ will be positive outward and the polar angle $\theta$ positive counterclockwise. Given the horizontal velocity components $v_{r}$ and $v_{\theta}$ along $r$ and $\theta$, the altimeter correction $D$, the acceleration of gravity $g$, and the time $t$, the cylindrical equations of motion are

$$
\dot{\mathrm{v}}_{\mathrm{r}}=\frac{\mathrm{v}_{\theta}{ }^{2}}{\mathrm{r}}+\mathrm{fv_{ \theta }}-\mathrm{g} \partial \mathrm{D} / \partial \mathrm{r}+\mathrm{F}_{\mathrm{r}}
$$




$$
\dot{\mathrm{v}}_{\theta}=-\frac{\mathrm{v}_{\theta} \mathrm{v}_{\mathrm{r}}}{\mathrm{r}}-\mathrm{f} \mathrm{v}_{\mathrm{r}}-\mathrm{g} \frac{\partial \mathrm{D}}{\mathrm{r} \partial \theta}+\mathrm{F}_{\theta}
$$

Here the dot denotes substantial time differentiation and $\mathrm{F}_{\mathrm{r}}$ and $\mathrm{F}_{\theta}$ are the components of the frictional force along $\mathrm{r}$ and $\theta$, respectively. $\dot{\mathrm{v}}_{\mathbf{r}}$ is positive when directed outward along $r$. Only the first of these two equations will be treated in view of the preceding statement of objective.

Given $\dot{\mathrm{v}}_{\mathrm{r}}=0, \mathrm{~F}_{\mathrm{r}}=0$, a type of gradient wind balance along $\mathrm{r}$ may be defined to be given by

$$
\frac{\frac{\mathrm{v}_{\theta}{ }^{2}}{\mathrm{r}}+\mathrm{fv}_{\theta}}{\mathrm{g} \frac{\partial \mathrm{D}}{\partial \mathrm{r}}}=1
$$

Defining

$$
\left.\dot{\mathrm{v}}_{\mathrm{r}}\right)_{\mathrm{gr}}=\dot{\mathrm{v}}_{\mathrm{r}}-\mathrm{F}_{\mathrm{r}}
$$

equation (1) may be written

$$
\left.\dot{v}_{r}\right)_{g r}=\frac{v_{\theta}^{2}}{r}+f v_{\theta}-g \frac{\partial D}{\partial r}
$$

The first calculation will be concerned with the question to what extent $\dot{\mathrm{v}}_{\mathrm{r}}{ }_{\mathrm{gr}}$ and also $\mathrm{fv}_{\theta}$ may be neglected compared to the centrifugal and pressure gradient terms. This calculation was performed for flight legs which were radial or which departed little from the radial direction. This restriction would not be required for storms with tangential symmetry. Such symmetry, however, was not observed.

Computational Procedure - - The computational procedure begins with the plotting of $\mathrm{v}_{\theta}$ and of " $\mathrm{D}$ " values $\mathrm{vs}$. radius on graph paper and the drawing of smooth curves of best fit through the data points (Figs. 7 and 8). 
Scattering of individual values was not great and representative curves were obtained in all cases for $\mathrm{v}_{\theta}$; however, there remained some leeway in drawing the "D" profiles, especially close to the center.

Next, the curves of $\mathrm{v}_{\theta}$ and " $\mathrm{D}$ " were divided into finite radial intervals starting at five nautical miles from the center, going outward. Integrating from $r_{1}$ to $r_{2}$ where $r_{2}>r_{1}, g \int_{r_{1}}^{r_{2}} d D / A r d r=g\left(D_{2}-D_{1}\right)$ where the total derivative has been substituted for the partial derivative. This is permissible since only a one-dimensional equation with $\mathrm{r}$ as independent variable is being solved.

Integration of the centrifugal acceleration is given by $\int_{\mathrm{r}_{1}}^{\mathrm{r}_{2}} \mathrm{v}_{\theta}{ }^{2} / \mathrm{r} \mathrm{dr}=\overline{\mathrm{v}_{\theta}{ }^{2}} \ln \mathrm{r}_{2} / \mathrm{r}_{1}$, where $\overline{\mathrm{v}_{\theta}{ }^{2}}$ is the mean value of $\mathrm{v}_{\theta}{ }^{2}$ between $\ln r_{1}$ and $\ln r_{2}$. This quantity is obtained by plotting $v_{\theta}{ }^{2}$ vs. In $\mathrm{r}$ and taking mean values between chosen intervals of $\ln \mathrm{r}$. Fig. 9 illustrates the method for graphical determination of $\overrightarrow{\mathrm{v}_{\theta}}$ between the 10 and $15 \mathrm{n}$ mi. radius.

The Coriolis acceleration is obtained by solving $\int_{r_{1}}^{r_{2}} f v_{\theta} d r=f v_{\theta}\left(r_{2}-r_{1}\right)$, where $\bar{v}_{\theta}$ is the mean of $v_{\theta}$ between $r_{2}$ and $r_{1}$ and $f$ is considered constant. Graphical determination of $\bar{v}_{\theta}$ is illustrated in Fig. ?.

The value of $f$ at the location of the storm center was used for each computation period. The greatest latitudinal extent of the computations were only $2-1 / 2^{\circ}$ latitude. This is equivalent to a maximum variation of $f$ at $25^{\circ}$ of only 9 percent. Since the magnitude of the Coriolis acceleration was usually much less than that of the centrifugal term--especially at high wind speeds near the center where $f \bar{v}_{\theta}$ was less than 5 percent of $\overline{v_{\theta}{ }^{2} / r}$-- the error in assuming $f$ constant over the whole storm area is negligible. Therefore it was permissible to use a single value for each flight. 
We may define

$$
g\left(D_{2}-D_{1}\right)=\overline{v_{\theta}^{2}} \ln \frac{r_{2}}{r_{1}}+f \overline{v_{\theta}}\left(r_{2}-r_{1}\right)
$$

where the subscript "gr" denotes the D-difference which the airplane should have observed with the wind field present (i.e.-in the case of $\left.\dot{\mathrm{v}}_{\mathrm{r}}\right)_{\mathrm{gr}}=0$ ). This result may be compared with the observed or actual $\mathrm{D}$-value gradient denoted by the subscript "act". We shall define

$$
g\left(D_{2}-D_{1}\right)^{\prime}=g\left(D_{2}-D_{1}\right)_{g r}-g\left(D_{2}-D_{1}\right)_{\text {act }}
$$

where the prime denotes the "unbalanced" portion of the D-difference. Then

$$
\begin{gathered}
\int_{r_{1}}^{r_{2}} \dot{v}_{r_{g r}} d r=+g\left(D_{2}-D_{1}\right)^{\prime}, \text { or } \\
\overline{\dot{v}_{r_{g r}^{\prime}}}=+g \frac{\left(D_{2}-D_{1}\right)^{\prime}}{r_{2}-r_{1}}
\end{gathered}
$$

The computations corresponding to the radial traverse along the eastern leg of Daisy on August 25 at $830 \mathrm{mb}$ are shown in Table III** Increments of $\mathrm{r}_{2}-\mathrm{r}_{1}$ were taken as 5 or $10 \mathrm{n}$. mi. from 5 to $20 \mathrm{n}$. mi. radius, and as $10 \mathrm{n}$. mi. farther out. The largest radius to which computations could be carried was 60 to $80 \mathrm{n}$. mi. in most cases. These distances are quite sufficient for a description of the acceleration field in the outer storm areas. Inside of the $10 \mathrm{n}$. mi. radius computations could not usually be made due to uncertainties of center location. Results in the interval from 10 to 15 miles are, at times, questionable for the same reason.

Along the flight leg illustrated in Table III, the gradient along $r$ of $\mathrm{D}_{\mathrm{gr}}$ exceeded that of $\mathrm{D}_{\text {act. }}$ along $\mathrm{r}$, so that $\overline{\dot{\mathrm{v}}}_{\mathrm{r}}$ ) $\mathrm{gr}$ was positive and directi ted outward. Profiles of both $\mathrm{D}_{\mathrm{gr}}$ and $\mathrm{D}_{\text {act }}$ are shown in Fig. 8 for the

\footnotetext{
D-values are given in feet as in the original data. Accelerations are expressed in knots/hour. They have not been converted to cgs units because it was felt that $1 \mathrm{knot} /$ hour imparts a feeling for the physical magnitudes involved more readily to most readers than $1.4 \times 10^{-2} \mathrm{~cm} \mathrm{sec}^{-2}$, its cgs equivalent.
} 
whole traverse from west to east side of Daisy on August 25. Note that while the slope of the $\mathrm{D}_{\mathrm{gr}}$ profile was greater than that of $\mathrm{D}_{\text {act }}$ in the east, the reverse was true in the west. The accumulated differences are substantial-about one third of the total $\mathrm{D}$-drop. Up to this point, the procedure has been nearly rigorous. Finally a more qualitative step was taken.

Computed values of $\overline{\dot{v}}_{\mathrm{r}}$ gr for each radial interval did not always yield smooth radial curves. This was caused partly by the integration intervals-i.e. only 5 to $10 \mathrm{n}, \mathrm{mi}$.--but primarily it was due to the limit of accuracy to which the gradient of " $D$ " could be measured. Whereas the winds are integrated over the radial interval of $r_{1}$ to $r_{2}$, the $D_{2}-D_{1}$ values are obtained by reading off values at individual radii $r_{2}$ and $r_{1}$. The original readings (by eye) of radio and pressure altimeters from films of the photopanel* cannot be considered to be more accurate than \pm 10 feet, so that the observed " $D$ " differences over small radial intervals cannot be regarded as exact, even when read from curves of best fit. In order to overcome this difficulty it was often necessary to increase the radial interval of integration to double or triple those chosen originally, especially at the inner radii, where tripling will increase the radial interval from 5 to $15 \mathrm{n}$. mi. This step, although possibly eliminating microscale features, does not obscure hurricanescale features.

Values of $\overline{\dot{\mathrm{v}}_{\mathrm{r}}}$ gr were plotted on maps at the mid-point of each radial interval $r_{2}-r_{1}$, and isolines were drawn for selected values. A grid was then placed over the analyzed $\left.\overline{\dot{v}}_{\mathrm{r}}\right)_{\mathrm{gr}}$ field in order to obtain averages around the storm at different radii. This averaging yields mean radial profiles of $\left.\overline{\dot{v}}_{\mathrm{r}}\right)_{\mathrm{gr}}$. It should be noted that interpolation by qualitative chart analysis was left to the very end and that all results not depending on this step are free from the subjectivity of line drawing.

The altimeters were not included in the automatic data punching system. 
Henceforth in this paper the symbol $\overline{\dot{v}}_{\mathrm{r}}$ gr $-i_{i}$ e. - the mean value of $\dot{\mathrm{v}}_{\mathrm{r}}{ }_{\mathrm{gr}}$ between the radial intervals $\mathrm{r}_{2}-\mathrm{r}_{1}-\cdots$-will be denoted just by the symbol $\dot{v}_{r}{ }_{\text {gr }}$ where the bar above $\dot{v}_{r_{g r}}$ gr has been deleted. Only when averaging is performed around the storm at selected radii will the bar over $\dot{v}_{\mathbf{r}^{\prime}}{ }_{\mathrm{gr}}$ be included to denote circular averaging.

Nearly all computations performed and conclusions drawn in this paper are based on data obtained from the ten levels listed in Table II. These levels were the ten best covered by National Hurricane Research Project aircraft during the 1956 through 1959 seasons---where reliable wind and D-values were obtained. 
1.-- Radial accelerations--relative to a fixed storm center.

Figures 12, 16, 20, 24, 28, 32, 36, 40,44, 48 portray the calculated values of $\left.\dot{\mathrm{v}}_{\mathrm{r}}\right)_{\mathrm{gr}}$ along the radial legs at the levels with the asterisk in Table I. All ten levels have large $\left.\dot{v}_{r}\right)_{g r}$ values, which shows that the radial gradient wind equation as here defined relative to the fixed storm center (equation 3) does not express the balance ofiforces along the radial direction. In nearly all cases, the areas of strongest winds were places of large positive $\left.\dot{v}_{\mathrm{r}}\right)_{\mathrm{gr}}$ and the areas of weakest winds places of large negative $\dot{v}_{r}{ }_{\text {gr }}$. Areas of positive $\dot{\mathrm{v}}_{\mathrm{r}^{\prime} \mathrm{gr}}$ were always found in the right quadrants* where the winds were the strongest and negative $\dot{\mathrm{v}}_{\mathrm{r}}{ }_{\mathrm{gr}}$ values are found in the left quadrants where the winds were the weakest. With symmetrical pressure distribution around the storm, these would be the proper places for the respective $\dot{v}_{r}{ }^{\prime}$ gr accelerations with an asymmetric wind field related, at least in part, to the storm's movement. However, this asymmetry in the wind field can seldom be accounted for by subtracting out the speed of the storm (Table II).

The order of magnitude of $\dot{\mathrm{v}}_{\mathrm{r}}$ gr was $10^{1}$ to $10^{2}$ knots/hour over most portions of the hurricane with range from zero to approximately 300 knots/hour. The average absolute maximum for all storm levels was between 100 and 200 knots/hour. These magnitudes are a large fraction of the radial pressure gradient or radial Coriolis and centrifugal accelerations. Figures 15, 19, 23, 27, 31, 35,39,43,47, 51 contain area distributions of the ratio $\left.\overline{\mathrm{v}}_{\mathrm{r}}\right)_{\mathrm{gr}} /\left(\mathrm{f} \overline{\mathrm{v}}_{\theta}+\overline{\mathrm{v}}_{\theta}^{2} / \mathrm{r}\right)$ for the ten levels with the asterisk in Table $\mathrm{I}$.

* The right front quadrant is the area in the $0^{\circ}$ to $90^{\circ}$ sector---when the direction of movement of the storm is taken as the zero degree line on a $360^{\circ}$ compass, counting clockwise. Other sectors have corresponding relations. 
It was unexpected that $\dot{\mathrm{v}}_{\mathrm{r}}{ }_{\mathrm{gr}}$ would be so large a fraction of the centrifugal plus Coriolis accelerations. In many places, especially in the western quadrants (Daisy August 25, $830 \mathrm{mb}$, both lower Cleo flights, and Carrie September 17), this ratio exceeded unity. There the radial pressure gradient force greatly exceeded the radial centrifugal and Coriolis acceleration.

Figures 52-55 portray mean radial profiles of $\left.\overline{\dot{v}}_{\mathrm{r}}\right)_{\mathrm{gr}} /\left(\mathrm{f} \overline{\mathrm{v}}_{\theta}+\overline{\mathrm{v}}_{\theta}{ }^{2} / \mathrm{r}\right)$ for all ten levels. There $\left.\overline{\dot{v}}_{\mathrm{r}}\right)_{\mathrm{gr}} /\left(\mathrm{f}_{\mathrm{v}_{\theta}}+\overline{\mathrm{v}}_{\theta}^{2} /\left.\mathrm{r}\right|_{\text {has been averaged around the }}\right.$ storm at $30^{\circ}$ intervals without respect to sign. There are rather large variations between the individual curves, but the storm levels varied in height, and the four storms differed in intensity, maturity, deepening tendency, etc. The large magnitudes of the ratios are, however, evident at all levels except on Daisy, August 27, $630 \mathrm{mb}$.

Fig. 56 contains a radial composite of $\left.\overline{\dot{v}}_{\mathrm{r}}\right)_{\mathrm{gr}} /\left[\mathrm{f} \overline{\bar{v}_{\theta}}+\bar{v}_{\theta}{ }^{2} / \mathrm{r} \mid\right.$ for all ten flights. There $\dot{\mathrm{v}}_{\mathrm{r}}{ }_{\mathrm{gr}}$ averages between 50 and 60 percent of the radial centrifugal and Coriolis accelerations. A similar composite of the integrated radial profile of $\overline{\dot{v}_{r}{ }_{g r r}} /|\overline{g \partial D / \partial r}|$ shows that $\dot{v}_{r_{g r}}$ averages 40 to 50 percent of the radial pressure gradient force. The difference between these two composites is due to the fact that the radial pressure force averaged 10 to 15 percent more than the radial Coriolis and centrifugal accelerations (to be discussed later). Figure 61 is a 10-level composite of the absolute values of $\left.\overline{\dot{v}}_{\mathrm{r}}\right)_{\mathrm{gr}}$, $\overline{\mathrm{g} \partial \mathrm{D} / \partial \mathrm{r}}$, and $\left(\overline{\mathrm{fv}}_{\theta}+\overline{\mathrm{v}_{\theta}^{2} / r}\right)$ plotted against radius. An area composite of

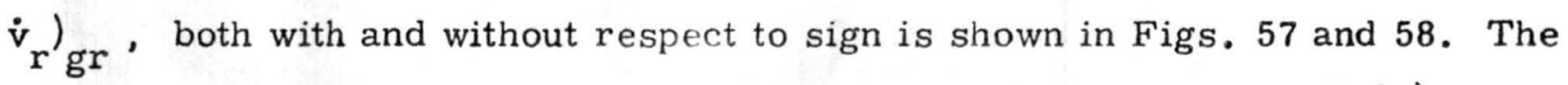
area composite with respect to sign clearly shows the asymmetry of $\dot{v}_{r}{ }^{\prime} g r$ bet ween right and left quadrants of the storm. All area compositing has been done with respect to the direction of motion of the storms.

\section{$1-b$ Radial accelerations---relative to the moving storm center ( ${ }^{\prime}{ }_{r r}$ ' gr $)$}

The above results have shown the radial accelerations relative to the instantaneous fixed point of the storm's center. Accelerations relative to the moving storm center will now be considered. 
By assuming that the storm moves at constant speed and direction, one can obtain these radial accelerations within the limits of accuracy of the data by subtracting the tangential component of the storm motion $\left(\mathrm{c}_{\theta}\right)$ from the total tangential wind component in equation 5. Other terms which arise from this change of reference to the moving storm center are less than 5 knots/hour and can be disregarded. This is over an order of magnitude lower than most of the computed $\dot{\mathrm{v}}_{\mathrm{rr}}{ }_{\mathrm{gr}}$ values. The acceleration of the storm displacement also enters into the calculations, but at the times of the calculations, the storms were moving at nearly constant direction. Changes in the rate of displacement were also small--less than one or two knots/hour. Thus the acceleration of the hurricane's movement was neglected.

With these omissions, the equation for the radial acceleration relative to the moving storm center---here denoted by $\left.\dot{\mathrm{v}}_{\mathrm{rr}}\right)_{\mathrm{gr}}$

$$
\begin{aligned}
& \left.\dot{\mathrm{v}}_{\mathrm{rr}}\right)_{\mathrm{gr}}=-\mathrm{g} \frac{\partial \mathrm{D}}{\partial \mathrm{r}}+\mathrm{f}\left(\mathrm{v}_{\theta}-\mathrm{c}_{\theta}\right)+\frac{\left(\mathrm{v}_{\theta}-\mathrm{c}_{\theta}\right)^{2}}{\mathrm{r}} \text {, or } \\
& \left.\dot{\mathrm{v}}_{\mathrm{rr}}\right)_{\mathrm{gr}}=\left(-\mathrm{g} \frac{\partial \mathrm{D}}{\partial \mathrm{r}}+\mathrm{f} \mathrm{v}_{\theta}+\frac{\mathrm{v}_{\theta}{ }^{2}}{\mathrm{r}}\right)-\left(\mathrm{fc}_{\theta}+\frac{2 \mathrm{v}_{\theta}^{\mathrm{c}}}{\mathrm{r}}-\frac{\mathrm{c}_{\theta}^{2}}{\mathrm{r}}\right)
\end{aligned}
$$

where $\dot{c}_{\theta}$ is the tangential component of the storm's motion. It is defined positive in the direction of the storm motion. $c_{\theta}$ is thus positive in the right quadrant and negative in the left quadrant. Substituting from equation 5 we obtain

$$
\begin{aligned}
& \left.\dot{\mathrm{v}}_{\mathrm{rr}}\right)_{\mathrm{gr}}+\left|\mathrm{fc}_{\theta}+\frac{2 \mathrm{v}_{\theta} \mathrm{c}_{\theta}}{\mathrm{r}}-\frac{\mathrm{c}_{\theta}^{2}}{\mathrm{r}}\right|=\left.\dot{\mathrm{v}}_{\mathrm{r}}\right|_{\mathrm{gr}} \text {, or } \\
& \dot{\mathrm{v}}_{\mathrm{rr}}{ }_{\mathrm{gr}}=\dot{\mathrm{v}}_{\mathrm{r}}{ }_{\mathrm{gr}}-\mathrm{M}
\end{aligned}
$$

where

$$
M=\left(\mathrm{fc}_{\theta}+\frac{2 \mathrm{v}_{\theta} \mathrm{c}_{\theta}}{\mathrm{r}}-\frac{\mathrm{c}_{\theta}{ }^{2}}{\mathrm{r}}\right)
$$

Rigorous verification of this has been accomplished from the absolute equations of motion in both vector and cartesian form. 
The term $\mathrm{fc}_{\theta}$, was always less than 5 knots/hour, hence had little effect on the calculations. The absolute value of $2 \mathrm{v}_{\theta}{ }_{\theta} / \mathrm{r}$ was usually 4 to 6 times greater than $\mathrm{c}_{\theta}^{2} / \mathrm{r}$. It is then the $2 \mathrm{v}_{\theta}{ }_{\theta}{ }_{\theta} / \mathrm{r}$ term that largely determines the value of M. Thus approximately

$$
\dot{\mathrm{v}}_{\mathrm{rr}}{ }_{\mathrm{gr}}=\dot{\mathrm{v}}_{\mathrm{r}}{ }_{\mathrm{gr}}-\frac{{ }^{2 \mathrm{v}_{\theta} \mathrm{c} \theta}}{\mathrm{r}}
$$

In general $\left.\dot{\mathrm{v}}_{\mathrm{rr}}{ }_{\mathrm{gr}}<\dot{\mathrm{v}}_{\mathrm{r}}\right)_{\mathrm{gr}}$. In the right quadrants, where $\dot{\mathrm{v}}_{\mathrm{r}}{ }_{\mathrm{gr}}$ is positive, $\mathrm{v}_{\theta}$ and $\mathrm{c}_{\theta}$ are also positive so that the convective term subtracts from $\dot{\mathrm{v}}_{\mathrm{r}}{ }_{\mathrm{gr}}$. In the left quadrants, with $\dot{\mathrm{v}}_{\mathrm{r}}{ }_{\mathrm{g}}$ gr negative, $\mathrm{v}_{\theta}$ positive and $\mathrm{c}_{\theta}$ negative, $\dot{\mathrm{v}}_{\mathrm{r}}{ }_{\mathrm{gr}}$ is also reduced. Since $v_{\theta}$ is greater in the right than in the left quadrants, the effect of the correction is strongest in the right quadrants. In the front and rear of the hurricane the correction, of course, is not present as ${ }_{\theta} \theta$ vanishes.

Values of $M$ were computed and inserted in equation 10. The resulting $\dot{\mathrm{v}}_{\mathrm{rr}}^{\prime}{ }_{\mathrm{gr}}$ patterns differed from those of $\left.\dot{\mathrm{v}}_{\mathrm{r}}\right)_{\mathrm{gr}}$ as follows.

1) The values of $\dot{\mathrm{v}}_{\mathrm{rr}}$ ' $_{\mathrm{gr}}$ were generally smaller than those of $\dot{\mathrm{v}}_{\mathrm{r}}$ ' gr ' being approximatelyr 60 to 80 percent of the $\dot{\mathrm{v}}_{\mathrm{r}}{ }_{\mathrm{gr}}$ values.

2) The positions of many of the $\left.\dot{\mathrm{v}}_{\mathrm{rr}}\right)_{\mathrm{gr}}$ centers were shifted from those of the $\dot{\mathrm{v}}_{\mathrm{r}}{ }_{\mathrm{gr}}$ centers.

3) Sometimes $\mathrm{M}$ exceeded $\dot{\mathrm{v}}_{\mathrm{r}}{ }_{\mathrm{gr}}$ in the right quadrants so that $\dot{\mathrm{v}}_{\mathrm{rr}}^{\prime}{ }_{\mathrm{gr}}$ became negative. In consequence, the area with negative $\dot{\mathrm{v}}_{\mathrm{rr}}^{\prime}{ }_{\mathrm{gr}}$ is very large compared with that of positive $\dot{\mathrm{v}}_{\mathrm{rr}}{ }_{\mathrm{gr}}$ on most of the levels analyzed.

Ten level area composites of $\dot{\mathrm{v}}_{\mathrm{rr}}{ }_{\mathrm{gr}}^{\mathrm{gr}}$ both with and without respect to sign are shown in Figs. 59 and 60. Again compositing has been done with respect to the direction of motion of each storm. The composite without respect to sign resembles the $\dot{\mathrm{v}}_{\mathrm{r}}$ ) $_{\mathrm{gr}}$ pattern in the essential features: maximum. accelerations to the right and left of the direction of motion, respectively 
(Fig. 60). This is an important result in that one might have expected the hurricanes to approach circular symmetry once the effect of center propagation was removed.

Figure 62 shows a radial composite plot of $\dot{\mathrm{v}}_{\mathrm{rr}}{ }^{\prime} \mathrm{gr}$ and $\dot{\mathrm{v}}_{\mathrm{r}}{ }_{\mathrm{gr}}$ without respect to sign. Although some subjectivity is inherent in the averaging it is felt that when averaging is extended over ten storm levels, the important broad scale features will stand out. It follows that neither the asymmetry nor the radial acceleration field itself is removed by defining the computation with respect to the moving center. Since most flignt levels were in the middle troposphere, where the net mass inflow or outflow are small, one might have thought that $\dot{\mathrm{v}}_{\mathrm{rr}}{ }_{\mathrm{gr}}$ should vanish here, especially when integrated around the storm. Such, however, is not the case.

We shall now return to the $\dot{\mathrm{v}}_{\mathrm{r}}{ }_{\mathrm{g}} \mathrm{gr}$ fields and study their characteristics in relation to other storm features.

2. Relation of the fields of $\dot{\mathrm{v}}_{\mathrm{r}}$ ) $\mathrm{gr}$ to $\mathrm{v}_{\mathrm{r}}$, and the maximum wind.

Analysis of the fields of the actual radial component of motion at all middle and lower tropospheric levels showed that in general there was a line dividing inflow and outflow iegions around the hurricane; further that this line usually closely bisected the areas of largest inward directed $\left.\dot{v}_{\mathrm{r}}\right)_{\mathrm{gr}}$ in the left quadrants and of largest outward directed $\dot{\mathrm{v}}_{\mathrm{r}}$ ) $\mathrm{gr}$ in the right quadrants (Figs. $12,14 ; 16,18 ; 20,22 ; 24,26 ; 28,30 ; 32,34 ; 36,38 ; 40,42 ; 44,46 ; 48,50)$.

In the right and left front quadrants of the storm, $v_{r}$ itself was predominantly directed outward. Inward directed motion occurred mainly in the rear quadrants. Thus in the right front quadrant $v_{r}$ and $\dot{v}_{r_{g r}}$ both pointed outward; in the left rear quadrant they both pointed inward. In the right rear quadrant, where the winds had inward radial components, this component decreased and reversed to the downstream side in agreement with the $\dot{v}_{\mathbf{r}}{ }^{\prime}$ gr 
field. The reverse was true for the left quadrant. This observation agrees with findings of a study by M. Ausman (1) who, working with surface ship data of many hurricanes, has shown that $v_{r}$ is directed inward in the rear quadrants and outward in the front quadrants at radii from $2^{0}$ to $6^{0}$ latitude from the centcr. From the present findings this appears to be valid over the whole hurricane area, not only at these outer radii, but also near the center.

It is an interesting fact that $v_{r}$ is zero approximately at the location of the maximum winds; there ito sign changes from negative to positive looking downstream. $\dot{v}_{\mathbf{r}}$ ) $g r$ is also positive and has maximum values very near this change from negative to positive $\mathrm{v}_{\mathrm{r}}$.

Qualitatively, this coincidence renders insight on the production of the maximum winds. In the region of weak winds in the left quadrants, air is accelerated inward and acquires a large crossing angle toward lower pressure. After some time the wind:speed increases sufficiently so that gradient balance in the radial direction is established $\left(\dot{v}_{r_{g r}}=0\right)$. At this point, however, the mass is still crossing towards lower pressure and gaining kinetic energy. It then overshoots the radius of gradient equilibrium and continues toward the center with $\left.\dot{\mathrm{v}}_{\mathrm{r}}\right)_{\text {gr }}$ directed outward. The speed increases until the air moves parallel with the contours of the isobaric surfaces. There the place of strongest winds and also the maximum $\left.\dot{\mathrm{v}}_{\mathbf{r}}\right)_{\mathrm{gr}}$ is reached. As the air then turns outward towards higher contours, both wind speed and $\dot{v}_{\mathbf{r}}$ gr again decrease.

3. Comparison of the Asymmetries of $g \partial D / \partial r$ and $\left(\overline{f v}_{\theta}+\overline{v_{\theta}^{2} / r}\right)$ around the storms.

The question may be asked whether the $\dot{v}_{r}$ gr field arises from asymmetries of the pressure gradient force, or of the $\left(f \bar{v}_{\theta}+\bar{v}_{\theta}{ }^{2} / r\right)$ distribution around the center. In order to answer this question, the fields of $D_{\text {act }}$ and $\mathrm{D}_{\mathrm{gr}}$ were drawn on maps. At selected radii, the averages of $\mathrm{D}_{\text {act }}$ and of $\mathrm{D}_{\mathrm{gr}}$ were determined. From a grid (Fig. 6) spaced evenly at $30^{\circ}$ intervals, the mean deviation from the average around the center was computed at each of the chosen radii. 
Figures 63-72 portray the results. Curves (a) show the deviation of $\mathrm{D}_{\mathrm{gr}}$ (i.e.the wind field), and curves (b) the deviation of $\mathrm{D}_{\text {act }}$ (i.e.-the pressure field). Though the curves on the different days vary considerably, certain features nevertheless stand out. At all levels, except Helene September $24,635 \mathrm{mb}$, the mean deviation of $\mathrm{D}_{\text {act }}$ never exceeded that of $\mathrm{D}_{\mathrm{gr}}$. Hence, asymmetry of the wind field in nine of the ten cases was then greater than that of the pressure field. This feature was especially prominent on three of the Daisy flights and in Cleo at $820 \mathrm{mb}$. This result does not obviously follow from the common knowledge that winds are stronger to the right than to the left of the direction of motion. One might have thought that the $\mathrm{D}_{\mathrm{gr}}$ field would vary concomitantly with $\mathrm{I}_{\text {act }}$. A radial composite for all 10 levels is seen in Fig. 73. The deviaticn of the pressure field varies little with radius, while the $\mathrm{D}_{\mathrm{gr}}$ deviations increase greatly towards the storm center. In the core of maximum winds the deviation of $\mathrm{D}_{\mathrm{gr}}$ exceeds that of $\mathrm{D}_{\text {act }}$ by a factor of three. Symmetry in the pressure field is produced as the heat released in ascending towers is swept around the center by the strong winds; and the

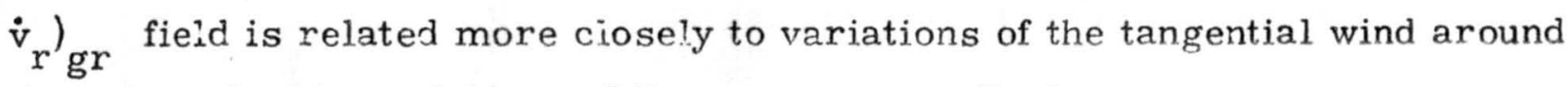
the storm than to variations of the pressure gradient.

4. Further Analysis of $\dot{v}_{r}{ }^{\prime} g r$ Field in Stationary Coordinates.

As the actual radial wind component is rarely observed to exceed 25 knots, and as individual air parcels often blow through areas of $\dot{\mathrm{v}}_{\mathrm{r}}{ }_{\mathrm{gr}}$ of 50 to 100 knots/hour for 30 to 60 minutes, it follows qualitatively that $\dot{\mathrm{i}}_{\mathrm{r}} \neq \dot{\mathrm{v}}_{\mathrm{r}}{ }_{\mathrm{gr}}$, at least over large portions of a hurricane. This interesting observation will now be analyzed by an attempt to compute $\dot{\mathrm{v}}_{\mathrm{r}}$ directly. In Eulerian coordinates

$$
\dot{v}_{r}=\frac{\partial v_{r}}{\partial t}+v_{\theta} \frac{\partial v_{r}}{r \partial \theta}+v_{r} \frac{\partial v_{r}}{\partial r}+w \frac{\partial v_{r}}{\partial z}
$$


Only the term $v_{r} \partial v_{r} / \partial r$ can be evaluated directly from the flight data; the range of magnitude was 1 to 10 knots/hour after integration over $10 \mathrm{n}$. mi. radial legs. Most values were less than $5 \mathrm{knots} / \mathrm{hour}$ however. The vertical advection term cannot be computed at all, but from general knowledge of vertical motion and vertical wind shear fields the order of magnitude should not exceed that of the radial advection, again after integration over $10 \mathrm{n} . \mathrm{mi}$. radial distances. The $w \partial v_{r} / \partial z$ term was thus neglected. For computing the tangential advection reliable $v_{\theta}$ fields are available through analysis; the weak link is $\partial \mathrm{v}_{\mathrm{r}} / \mathrm{r} \partial \theta$ which was obtained by tabulating the analysis of $\mathrm{v}_{\mathrm{r}}$ prescribed in this paper on the grid of Fig. 6. In spite of the weakness of the computation, systematic features of the tangential advection were obtained and reliable features should be revealed by the ten cases. The calculated magnitude of this term had the order of 10 knots/hour with range from 0 to 50 knots/hour. The quantity $\partial v_{r} / \partial t$ was determined by moving the $v_{r}$-field across the fixed grid of Fig. 6 with the direction and speed of the storm motion. For the fast moving storms changes were computed from one-half hour before to onehalf hour after the time when the $v_{r}$-field center position was connected with the fixed grid center. For slower moving systems the time interval chosen for a / at was from one hour before to one hour after the time when the center position corresponded with the fixed grid center.

$$
\text { initial expectation was that } \dot{\mathrm{v}}_{\mathrm{r}}{ }_{\mathrm{g} g r}=\partial \mathrm{v}_{\mathrm{r}} / \partial t+\mathrm{v}_{\theta} \partial \mathrm{v}_{\mathrm{r}} / \mathrm{r} \partial \theta+\mathrm{v}_{\mathrm{r}} \partial \mathrm{v}_{\mathrm{r}} / \partial \mathrm{r}
$$
within computational limits. This proved not to be correct by wide margins. The Daisy flight of August 27 at $630 \mathrm{mb}$ provides a typical illustration. Fig. 74 contains the field of $\dot{\mathrm{v}}_{\mathrm{r}}$, to be compared with Fig. 20. Only 40-60 percent of the $\dot{\mathrm{v}}_{\mathrm{r}}{ }_{\text {gr }}$ field are accounted for by $\dot{\mathrm{v}}_{\mathrm{r}}$. Broadly speaking, the orientation of the patterns and center locations are in fair agreement. Fig. 75 contains the difference field. From the earlier definition

$$
\left.\mathrm{F}_{r}=\dot{\mathrm{v}}_{r}-\stackrel{\circ}{\mathrm{v}}_{\mathrm{r}}\right)_{\mathrm{gr}}
$$


it must now be postulated that Fig. 75 represents the $\mathrm{F}_{\mathrm{r}}$ field after subtraction of Fig. 20 from Fig. 74 in the sense of equation 13. Admittedly this postulated force field is exceedingly strong $\left(10^{-1}\right.$ to $3 \times 10^{0}$ dynes/unit mass $)$. But in a strongly turbulent system such as the hurricane, the existence of such strong frictional forces should not be rejected a priori. Further comments on the magnitude of $\mathrm{F}_{\mathrm{r}}$ will be offered shortly.

Fig. 76 contains radial profiles of $\dot{\mathrm{v}}_{\mathrm{r}}{ }_{\mathrm{gr}}, \mathrm{F}_{\mathrm{r}}$, and $\dot{\mathrm{v}}_{\mathrm{r}}$ averaged around the storm at $10 \mathrm{n}$. mi. intervals for Daisy August 27,630 mb. We see that $\mathrm{F}_{\mathrm{r}}$ and $\dot{\mathrm{v}}_{\mathrm{r}}$ are nearly equal and average over half the $\dot{\mathrm{v}}_{\mathrm{r}}{ }_{\mathrm{gr}}$ values. That $\left|\mathrm{F}_{\mathrm{r}}+\dot{\mathrm{v}}_{\mathrm{r}}\right|>\left|\dot{\mathrm{v}}_{\mathrm{r}}{ }_{\mathrm{gr}}\right|$ at all radii is due to the lack of exact superpositioning of $\dot{\mathrm{v}}_{\mathrm{r}}$ 'gr and $\dot{\mathrm{v}}_{\mathrm{r}}$ centers.

Similar properties were obtained for the other hurricanes so that it was considered permissible to form the average of all ten cases, Figs. 61, 77, 78. Here also, $\mathrm{F}_{\mathrm{r}}$ and $\dot{\mathrm{v}}_{\mathrm{r}}$ are nearly equal. $\mathrm{F}_{\mathrm{r}}$ approaches 85 percent of $\stackrel{\mathrm{v}}{\mathrm{r}}_{\mathrm{gr}}$ at radii from 30 to $60 \mathrm{n}$. $\mathrm{mi}$., and 70 percent over the whole radial distance. Since $\dot{v}_{r}{ }_{g r}$ itself was a large fraction of $g \partial D / \partial r$, it follows that frictional and pressure gradient forces have roughly the same magnitude, at least over substantial portions of a hurricane.

In one respect, Daicy August 27,630 mb was not typical, namely that it was the only level studied with net positive $\dot{\mathrm{v}}_{\mathbf{r}}{ }^{\prime}$ gr values after integration around the center. The balancing frictional force had to be directed inward. Daisy on the 27 th had reached maximum intensity, its winds were slightly

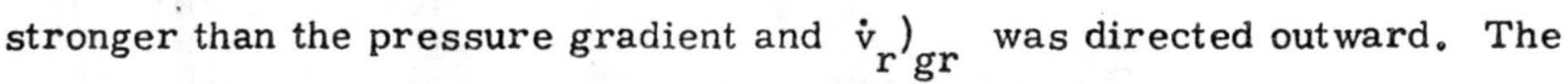
storm was just beginning to fill at this time. In eight of the other nine cases, the pressure gradient force exceeded the centrifugal plus Coriolis acceleration-in the integral around the hurricanes. The net $\left.\dot{\mathrm{v}}_{\mathrm{r}}\right)_{\mathrm{gr}}$ field consequently was directed inward and $\mathrm{F}_{\mathbf{r}}$ outward, 


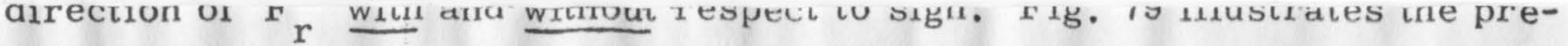
dominance of outward directed $F_{r}$ especially well. Figs. 81 and 82 portray 10 level area composites of $\dot{\mathrm{v}}_{\mathrm{r}}$ both with and without respect to sign. It is clearly evident that the values of $\dot{v}_{r}$ are not of great enough magnitude to account for the $\dot{v}_{r}{ }_{\text {gr }}$ accelerations even when individual storm level superpositioning of these two fields is eliminated by the ten level average.

The above calculations have determined the $\mathrm{F}_{\mathrm{r}}$ accelerations relative to an instantaneous fixed point of the storm center. The frictional acceleration with respect to the moving storm center (denoted $\mathrm{F}_{\mathrm{rr}}$ ) is identical with that with respect to the instantaneous fixed center when the storm propagation is assumed constant *

5. Preliminary Comments on the Magnitude of $\mathrm{F}_{\mathrm{r}}$.

From the discussions of Osborne Reynolds (14) it appears possible to represent $\mathrm{F}_{\mathrm{r}}$ by the Reynolds form of eddy stresses. In cylindrical coordinates, $\mathrm{F}_{\mathrm{r}}$ then has four components,

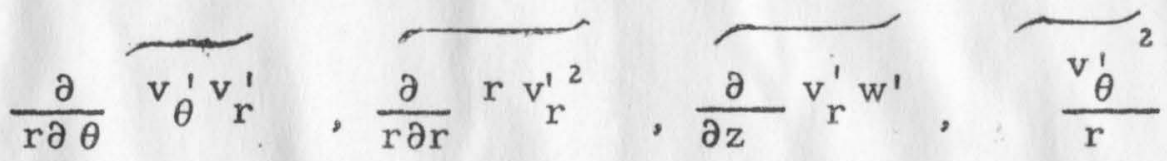

where the wavy bar denotes area average and the prime represents deviations from this mean. In this form the term $\partial / \partial z \quad v_{r}^{\prime \prime} w^{\prime}$ would be the vertical gradient of the horizontally integrated product of the radial and vertical wind eddies.

Evaluations of $v_{r}^{\prime}$ and $v_{\theta}^{\prime}$ values and their tangential and horizontal gradients was made on a number of the mid-tropospheric levels here studied from continuous pitot tube recordings mounted on the aircraft. These pitots measure small changes in atmospheric pressure as the aircraft travels along

* See Appendix for clarification of this point. 
the radial and tangential legs. These minute pressure changes are converted into their equivalent eddy wind variations. From such information, the Reynolds stresses can be computed.

From the preliminary computations $\frac{\partial \mathrm{v}_{\theta}^{\prime} \mathrm{v}_{r}^{\prime}}{\mathrm{r} \partial \theta}, \frac{\partial \mathrm{rv}_{\mathbf{r}}^{\prime 2}}{\mathrm{r} \partial \mathrm{r}}$ and $\frac{\mathrm{v}_{\theta}^{\prime 2}}{\mathrm{r}}$ turn out to be quite small and cannot account for $\mathrm{F}_{\mathrm{r}}$. An order of magnitude estimate of the $\partial / \partial z v_{r}^{\prime} w^{\prime}$ term, using determined values of $v_{r}^{\prime}$ from the pitot tubes and assuming Malkus' (10) and Riehl's* calculations of velocities in hurricane $\mathrm{Cb}$ 's, indicate that this term may have the required magnitude. Vertical gradients of $\mathrm{v}_{\mathrm{r}}^{\prime} \mathrm{w}^{\prime}$ were determined with a two-layer model, i.e. estimates were made for the mid-tropospheric levels and zero values were assumed for the surface and the tropopause. This is a crude approximation, but it encourages one to seek further experimental data. With improved sampling and measuring techniques a reliable estimate of the importance of the $\partial / \partial z \widetilde{v_{r}^{\prime} w^{\prime}}$ term can be achieved. New instrumentation and special flight planning with National Hurricane Research Project aircraft is being contemplated for the 1961 and 1962 seasons with this purpose in mind.

\section{Radius of Trajectory Curvature}

The gradient wind equation in natural coordinates on a constant pressure surface is

$$
0=-g \partial D / \partial n+f V+\frac{V^{2}}{R}
$$

where $\mathrm{V}$ is the wind speed

$\mathrm{n}$ is distance measured perpendicular to $\mathrm{V}$, and

$\mathrm{R}$ is the radius of trajectory curvature.

* Calculations performed in U. of Chicago meteorology laboratory class given by Prof. H. Riehl (1960). 
As mentioned in the introduction, this equation is difficult to solve since $R$ is not observed directly; moreover, $\partial \mathrm{D} / \partial \mathrm{n}$ also is not always obtainable . However, at places where $v_{r}=0 n$ is along $r, \partial D / \partial n=\partial D / \partial r$, and $\mathrm{y}=\mathrm{v}_{\theta}$. At these locations $\mathrm{v}_{\theta}{ }^{2} / \mathrm{R}+\mathrm{fv}_{\theta}=\mathrm{g} \partial \mathrm{D} / \partial \mathrm{r}$, and

$$
R=\frac{\mathrm{v}_{\theta}^{2}}{\mathrm{~g} \frac{\partial \mathrm{D}}{\partial \mathrm{r}}-\mathrm{fv}_{\theta}}
$$

The radius of trajectory curvature, as given by equation 15 can now be computed directly along the lines of $\mathrm{v}_{\mathrm{r}}=0$ and may be compared with radii determined from kinematic methods. It is apparent that in view of the possible importance of frictional forces, deduced earlier, this comparison should not succeed. $R$ was evaluated with equation 15 and kinematically with Blaton's formula for many places of the ten storm levels where $v_{r}=0$ occurred along or close to a flight leg. As expected, $R$ as given by equation 15 was consistently too small to the left and too large to the right of the direction of motion. If equation 15 is amended to include radial friction we have

$$
\frac{v_{\theta}^{2}}{R}+{ }_{f v}=g \frac{\partial D}{\partial r}-F_{r}
$$

Addition of equation 16 with the earlier obtained equation, i.e.--

$$
\begin{array}{ll}
\dot{\mathrm{v}}_{\mathrm{r}}-\frac{\mathrm{v}_{\theta}^{2}}{\mathrm{r}}-\mathrm{fv}_{\theta}=-\mathrm{g} \frac{\partial D}{\partial \mathrm{r}}+\mathrm{F}_{\mathrm{r}} & \text {, yields } \\
\frac{\mathrm{v}_{\theta}^{2}}{\mathrm{R}}-\frac{\mathrm{v}_{\theta}^{2}}{\mathrm{r}}+\dot{\mathrm{v}}_{\mathrm{r}}=0 & \text {, or } \\
R=\frac{\mathrm{v}_{\theta}^{2}}{\frac{\mathrm{v}_{\theta}^{2}}{r}-\dot{\mathrm{v}}_{\mathrm{r}}} &
\end{array}
$$


This formulation should yield correct values. Computed radii of trajectory curvature with equation 15 often are as low as 7-8 miles to the left and as large as 35 miles to the right of the direction of motion at radii of 20 miles from the center. Comparable values obtained with equation 17 are 12 and 27 miles respectively. These values obtained with equation 17 agree much better with the kinematically determined radii from Blaton's formula. Especially in the left quadrants of the storm is this discrepancy between equation 15 and 17 large.

Another approach, showing the non-validity of the gradient wind assumption in natural coordinates is to assume $\partial \mathrm{D} / \partial \mathrm{n}=\partial \mathrm{D} / \partial \mathbf{r}$ in the areas where $\dot{\mathrm{v}}_{\mathrm{r}}$ ) $\mathrm{gr}$ is negative and compute the values of $R$ necessary for balance from equation $V / R^{2}+f V=g \partial D / \partial r$, or

$$
R=\frac{V^{2}}{g \frac{\partial D}{\partial r}-f V}
$$

In practically all places where this calculation was performed values of $R$ necessary for gradient wind balance in natural coordinates were. smaller than the values of $R$ determined kinematically by Blaton's formula or with equation 17.

This method has the advantage that it can be applied at places where $v_{r} \neq 0$. The assumption $\partial \mathrm{D} / \partial \mathrm{n}=\partial \mathrm{D} / \partial \mathrm{r}$ where $\partial \mathrm{D} / \partial \mathrm{n}$ always $\geq \partial D / \partial r$ is entirely permissible at places where $\dot{\mathrm{v}}_{\mathrm{r}}{ }_{\mathrm{gr}}$ is negative. This assumption decreases rather than increases the difference between the $R$ gotten from equations 15 and 17 . Thus substitution of $\partial D / \partial r$ for $\partial D / \partial n$ in equation 14 increases $R$ and causes computed values of $R$ under the gradient wind assumption in natural coordinates to compare more favorably with kinematic and equation 17 determinations. Even with this substitution the computed differences in $R$ in the frictional and nonfrictional cases are appreciable. 
It follows that the gradient or cyclostrophic assumption, usually made in hurricanes can be in error by as much as 50 to 100 percent. One cannot hope to obtain the proper constraints in hurricane growth and maintenance with the cyclostrophic approach.

7. a--Condensation Heating Related to Radial Friction and Deepening Tendency

If one averages the $D_{\text {act }}$ values around the storm at constant radius at the grid points shown in Figure 6 and plots these $\mathrm{D}_{\text {act }}$ values with respect to radius at $10 \mathrm{n}$. mi. intervals he obtains a radial profile of the actual " $\mathrm{D}$ " values as discussed at the beginning of the report. A similar profile of the $\mathrm{D}_{\mathrm{gr}}$ values can also be made. If the two profiles are plotted together beginning at a uniform value at an outer reference radius, say 80 or $100 \mathrm{n}$. mi., the accumulative inward imbalance between the $\mathrm{D}_{\text {act }}$ and $\mathrm{D}_{\mathrm{gr}}$ fields is portrayed (Fig. 83). The 80 to $100 \mathrm{n}$. mi. reference radius is a reasonable starting point, since the major condensation warming occurred inside of this limit in all cases.

This integrated height difference between the $D_{\text {act }}$ and $D_{g r}$ profiles can be converted into a virtual temperature excess or deficit with the hydrostatic equation from the tropopause region downward, holding a high pressure surface, say $100 \mathrm{mb}$, constant. A positive integrated value of $\left|\mathrm{D}_{\mathrm{gr}}-\mathrm{D}_{\text {act }}\right|$ means that the atmosphere is warmer than would be computed with the assumption $\left.\dot{v}_{r}\right)_{g r}=0$. A negative value of $\left|D_{g r}-D_{\text {act }}\right|$ has the opposite meaning.

Heating of the air beyond that demanded for radial balance of forces will be denoted as "excess heating." Part of this heating, if present, is used to maintain the storm against frictional dissipation; another part is available to intensify the storm. There is thus a unique balance between frictional 
dissipation, intensification, and excess or deficit heating when values integrated around a hurricane are considered. Thus

$[$ EXCESS HEATING $]-[$ FRICTIONAL DISSIPATION $]=\left[\begin{array}{c}\text { STORM } \\ \text { INTENSIFICATION }\end{array}\right]$

If the integrated value of $\left.\mid \mathrm{D}_{\mathrm{gr}}-\mathrm{D}_{\text {act }}\right)$ from the outer to the inner storm radius is negative, then not enough condensation heat is released to maintain the pressure gradient at the value needed to go with the wind pattern even in the frictionless case, and the storm must decay.

Of the ten levels analyzed, eight had large positive integrated values of $\left(D_{g r}-D_{\text {act }}\right)$ i.e.---strong excess heating. These hurricanes were deepening or moving in steady-state. The other two cases (Daisy August 27,630 mb, August $28,620 \mathrm{mb})$ : had slightly negative and slightly positive $\left(\mathrm{D}_{\mathrm{gr}}-\mathrm{D}_{\text {act }}\right)$ values, respectively when averaged around the storm. The condensation heating, although large, that was occurring in Daisy on the 27th and 28th had no large residual to balance the dissipation of kinetic energy by friction. It was on the 27th that Daisy reached maximum intensity and began to fill. On the 28th Daisy was filling rapidly. Retarding frictional effects thus overweighed the generating effects of condensation heating on these two days.

Figures 84 and 85 show the predominance of negative $\dot{\mathrm{v}}_{\mathrm{r}}{ }_{\mathrm{gr}}$ and $\dot{\mathrm{v}}_{\mathrm{rr}}{ }^{\prime}{ }_{\mathrm{gr}}$ over positive values of these quantities. Negative values of $\dot{\mathrm{v}}_{\mathbf{r}}{ }_{\mathrm{gr}}$ and $\dot{\mathrm{v}}_{\mathrm{rr}}^{\prime}{ }_{\mathrm{gr}}$ correspond to "excess heating" and are proportional to positive integrated values of $\left(D_{g r}-D_{\text {act }}\right)$ from outside to inside of the storm, as shown by equations 8 and 9 when integration is performed in a negative sense along $r$.

These composite radial profiles, which include Daisy levels on August 27 and 28 , bring out clearly the considerable excess of the observed $D$-value drop from outside to inside of the hurricanes above that required to 
balance centrifugal plus Coriolis accelerations when composites of the ten levels is made. In these diagrams $\left|\dot{\mathrm{v}}_{\mathrm{r}^{\prime}{ }_{\mathrm{gr}}}\right|$ and $\left|\dot{\mathrm{v}}_{\mathrm{rr}^{\prime}{ }_{\mathrm{gr}}}\right|$ have been averaged around the storm without respect to sign (top curve) and divided into positive and negative parts. The major contribution to $\left|\dot{\mathrm{v}}_{\mathrm{r}_{\mathrm{gr}}}\right|$ and $\left|\dot{\mathrm{v}}_{\mathrm{rr}}{ }_{\mathrm{gr}}\right|$ is clearly seen to be made up of negative values of these quantities----i.e--positive $\left(D_{g r}-D_{\text {act }}\right)$ or excess of pressure gradient over centrifugal and Coriolis acceleration. At some levels, notably the two Carrie flights (Figs. 36 and 40) negative $\dot{\mathrm{v}}_{\mathbf{r}_{\mathrm{grr}}{ }_{\mathrm{gr}}}$ or $\dot{\mathrm{v}}_{\mathrm{rr}}{ }_{\mathrm{gr}}$ values prevailed over nearly all of the flight level.

These large residual negative $\dot{\mathrm{v}}_{\mathrm{r}}$ ) $\mathrm{gr}$ accelerations are needed to maintain the storm against frictional dissipation and to deepen it. It is no coincidence that the two levels which showed little negative residual of $\dot{\mathrm{v}}_{\mathrm{r}}{ }_{\mathrm{gr}} \mathrm{gr}$ were the two where filling was beginning or taking place. Also it is no coincidence that steady state storms must have negative integrated values of $\dot{\mathrm{v}}_{\mathbf{r}} \mathbf{g r}_{\mathrm{gr}}$ to balance frictional dissipation.

7. b--Condensation Heating Related to the Wind and Pressure Field on August 25.

Daisy on August 25th was in the deepening stage (Fig. 1-2). Radial legs were flown along four distinct levels on this day $(950,830,570,237 \mathrm{mb})$. An excellent and unique opportunity thus exists to obtain vertical gradients of the computed $\left.\dot{\mathrm{v}}_{\mathbf{r}}\right)_{\mathrm{gr}}$ fields and perhaps obtain some insight into the deepening process that was occurring on this day. At first, vertical thickness values were determined from the actual slopes of the isobaric surfaces and then converted to mean virtual temperature with the hydrostatic equation. Taking the mean virtual temperature in each layer at $r=100 \mathrm{n}$. mi. as reference temperature, the inward warming was computed over intervals of $20 \mathrm{n}$. miles and plotted accumulatively from $100 \mathrm{n}$. mi. to the center (Fig. 87). It is seen that nearly all warming took place inside the $40 \mathrm{n}$. mi. radius. The computed virtual mean temperature in the eye between 570 and $237 \mathrm{mb}$ was $4-1 / 2^{\circ} \mathrm{C}$ warmer than the temperature in the same layer at the $100 \mathrm{n}$. mi. radius. 
This computed cross-section agrees quite well with that obtained from the aircraft temperatures (Fig. 88). Figures 87 and 88 corroborate a previous evaluation by Malkus (5), who, using the same data, had found that a good approximation to the $\mathrm{D}$-field in the low troposphere could be obtained by assuming an undisturbed top in the high troposphere and integrating downward hydrostatically with the observed (virtual) temperatures.

We shall now: approach the interesting question as to. what fraction of this total temperature field is due to "excess" heating as defined above. For this purpose the $\left(D_{g r}-D_{\text {act }} \mid\right.$ profiles were plotted together on one diagram. If we set $\left(D_{\text {gr }}-D_{\text {act }}\right)=0$ at the storm periphery and then plot $\left|D_{\text {gr }}-D_{\text {act }}\right|$ as a function of radius at each level, the unbalanced portion of the drop in height of the pressure surfaces will become readily apparent.

At the lower three pressure levels, the radial slope of the actual D-profile---after integrating around the hurricane at each level---exceeded that of the $D_{g r}$ profile. At $237 \mathrm{mb},\left(\mathrm{f}_{\mathrm{v}_{\theta}}+\overline{\mathrm{v}_{\theta}{ }^{2} / \mathrm{r}}\right)$ exceeded $\mathrm{g} \partial \mathrm{D} / \partial \mathrm{r}$. But $\left(\mathrm{D}_{\mathrm{gr}}-\mathrm{D}_{\text {act }}\right)$ integrated over the entire storm volume was negative. The four radial legs used for computation at $237 \mathrm{mb}$ are shown in Figure 11 . It was not possible to obtain values of $\left.\dot{\mathrm{v}}_{\mathrm{r}}\right)_{\mathrm{gr}}$ within the $20 \mathrm{n}$. mi. radius, and radial symmetry over a limited distance had to be assumed for computations along legs $\mathrm{D}$ and $\mathrm{B}$. Nevertheless, the cross-hatched part of the curve in Figure 86 is believed to be reasonably accurate.

Vertical changes in the profile of $\left|D_{g r}-D_{\text {act }}\right|$ and hence also $\left.\dot{v}_{r}\right)_{g r}$ when integrated around the storm, were quite small from 950 to $570 \mathrm{mb}$. The greatest vertical change in the profiles occurred between 570 and $237 \mathrm{mb}$ (Fig. 86).

The portion of the inward temperature increase arising from the $\left(\mathrm{D}_{\mathrm{gr}}-\mathrm{D}_{\text {act }}\right)$ profiles are shown in Figs. 89 and 90 in profiles form. As noted earlier, computations as illustrated in Figs. 86 to 90 have a less secure basis than those performed along individual radial legs because they are 
dependent on field analysis by qualitative methods and subsequent integration around the center. If, however, it will be granted that no serious analysis error has been made, a quite interesting result has been obtained. Figures 89 and 90 may be interpreted as showing the heating function which gives rise to storm development and to circulation maintenance against internal and surface friction.

The "free" surface pressure fall at the ground, estimated from Figs. 89 to 90 , is about 4 to $5 \mathrm{mb}-$-with the assumption of an undisturbed top at 50,000 feet. Some of this pressure fall must, by previous reasoning, be used up in internal and surface frictional dissipation. The amount of heating that is left over after this dissipation is used to intensify the storm. In this case of Daisy on the 25th, internal and surface frictional dissipation was smaller than the excess heating. It also follows that since development of the wind field lagged the pressure field, that the drop in central pressure was the primary feature and occurred before intensification of the hurricane winds.

It would appear that, from the local thermodynamic viewpoint, the action of the cumulus clouds in the initial disturbance must have been such as to produce a net area warming of the troposphere above $500 \mathrm{mb}$, with magnitudes of $1^{\circ} \mathrm{C}$ above that ordinarily cbtained from convection in disturbances.

If Daisy, as observed on August 25, is considered typical of developing hurricanes, then a postulation of initial warming in the middle and especially in the upper troposphere is a definite initial condition of hurricane development. In this connection, it is of interest to record that a reconnaissance plane of the U.S. Navy which scouted the cyclogenetic area about 12 hours before the research missions reached Daisy (2200-2400 GMT, August 24), observed a nearly solid precipitation area on radar with extent of about $10^{4} \mathrm{n}$. $\mathrm{mi}^{2}{ }^{2}$ and convective echo tops above 40,000 feet in the precise area where Daisy was forming. 
The principal results from all of the levels within the four storms studied is that:

1. The gradient wind equation along the radial direction, as defined in this paper for hurricanes, does not express the radial balance of forces to a satisfactory degree of approximation in nearly all portions of the storm. The quantity $\left.\dot{\mathrm{v}}_{\mathrm{r}}\right)_{\mathrm{gr}}$ is nearly always a large fraction of the Coriolis and centrifugal acceleration. In some places $\dot{\mathrm{v}}_{\mathrm{r}}{ }_{\mathrm{gr}}$ is even larger than these accelerations.

2. Internal radial friction plays an important role in balancing the cyclindrical radial equation of motion. The frictional acceleration is directed towards the storm center when $\dot{\mathrm{v}}_{\mathrm{r}}{ }_{\mathrm{gr}}$ is positive and outward from the storm center when $\dot{\mathrm{v}}_{\mathrm{r}}{ }_{\mathrm{gr}}$ is negative. The average magnitude of the radial frictional acceleration for the 10 levels for which area integration could be performed is 25 to 30 percent of the pressure gradient force and 35 to 40 percent of the Coriolis and centrifugal acceleration. These internal radial frictional accelerations may possibly be accounted for by a vertical Reynolds stress term.

3. All deepening or steady state hurricanes must have stronger pressure gradients than centrifugal and Coriolis accelerations in order to account for frictional dissipations. 


\section{CONCLUSION}

In this study the balance of forces in hurricanes has been examined along the radial direction in stationary and moving coordinates; further, the radius of trajectory curvature has been computed in selected portions of ten flight missions. The Coriolis acceleration proved negligible compared to the centrifugal acceleration as expected. But gradient or cyclostrophic balance in general is not achieved by a wide margin, both along the radial axis and in natural coordinates. The pressure gradient far exceeds that required to balance the centrifugal acceleration to the left, and falls somewhat short of the requirement to the right of the direction of motion. In moving coordinates this asymmetry is reduced but by no means eliminated. Hence the maximum wind may be interpreted as produced by "overshooting" toward low pressure far beyond the point of equilibrium. A similar situation appears to exist with respect to many extratropical wind maxima, especially high-speed centers along jet streams.

The field of radial motion shows general outflow ahead and inflow behind the hurricane. The zero line is to right and left of the direction of motion, approximately where the maximum difference between pressure gradient force and centrifugal acceleration is found. This geometric relation is considered more than a coincidence and supports the preceeding deductions about the origin of the maximum wind.

The air is accelerated radially in the sense prescribed by the difference between pressure gradient force and centrifugal acceleration. But the magnitude of the actual acceleration generally falls short of the computed acceleration by a wide margin. Hence it becomes necessary to postulate the existence of large lateral frictional forces which act opposite to the ${ }^{\prime} \mathbf{~}^{\prime} \mathbf{g r}$ accelerations. The magnitude is $10^{-1}$ to $10^{\circ}$ dynes per unit mass which corresponds to a pressure gradient force of $1 \mathrm{mb} / 5$ miles. Such large frictional forces 
previously have not been suspected to exist. Nevertheless, there appears to be no valid reason a priori why the friction should not rise to become a substantially larger fraction of the pressure gradient force in hurricane-type systems than under normal atmospheric circumstances. If so, an important break on hurricane development has been uncovered, and it remains for the future to give a quantitative expression to enter as a constraint in hurricane models.

A few initial steps were taken to relate dynamics and energetics. From the difference between pressure gradient force and centrifugal acceleration, the departure of central pressure from that required to balance the wind field can be computed, also the temperature field which must be assumed with the pressure departure, assuming hydrostatic conditions with a top of the disturbance at $100 \mathrm{mb}$. Only one case had sufficient flight levels to perform all of these calculations.

In this instance there was a "forced" surface pressure drop of 4- $5 \mathrm{mb}$ and an "excess" temperature warming about $1^{\circ} \mathrm{C}$, usually in the hightropospheric layer above $500 \mathrm{mb}$. From this it appears that hurricane development is related to the capacity of the atmosphere to effect a large heat transport into the upper atmosphere which from heat balance considerations, must be effected by ascent in narrow channels as demonstrated by Riehl and Malkus, 1958, (18) for the equatorial trough zone. The relation between dynamics and energetics has merely been touched on in this paper, but one definite suggestion from this aspect of the work is that one should search for conditions in tropical depressions which favor banding the mass ascent into narrow walls or lines. 


\section{ACKNOWLEDGMENTS}

The author wishes to express his sincere graditude to Professor Herbert Riehl under whose direction this paper was written. Professor Riehl proposed this topic, encouraged, and supervised all phases of its carrying out. The author also wishes to thank the National Hurricane Research Project and its past and present directors Messrs. Robert Simpson, Cecil Gentry, and Harry Hawkins for generously furnishing the valuable data needed to make this study possible. The author expresses his appreciation to the U.S. Weather Bureau for providing financial assistance and to Mrs. Della Friedlander for drafting the illustrations. Thanks are also due to Dr. T. N. Krishnamurti of the U. of Chicago and the above mentioned Mr. Robert Simpson for helpful discussions on this subject matter. 
A P PENDIX

$-34-$ 


\section{APPENDIX}

\section{Reliability of the Computations}

The reader's first reaction to this study may be tinged with some skepticism as to the accuracy of the data and of the reliability of some of the computations made from the qualitative analysis. This may be particularly true of computations that made use of the radial wind component.

It must be emphasized that the Doppler navigation system, from which all the winds were obtained, is an extremely accurate navigation instrument. Winds above 10 to 15 knots are accurate to within two to three percent in both direction and speed--except at isolated points in turn and in a few heavy rain areas. These areas were not used in the computations. The tangential wind components are just as accurate as the total wind.

All computations where " $\mathrm{D}$ " values were used were dependent only on the gradient of the " $D$ " values and not on the absolute values themselves.

It shoud be remembered that the radial winds used in this study were those of the actual radial wind components and not the relative radial wind components. The magnitudes of the actual radial winds are much greater than the relative radial winds and lend themselves to far better evaluation.

Computations using the actual radial winds were made only from careful field analysis where isolated non-representative values could be disregarded and a relatively smooth well defined pattern could be obtained. Evaluation of $\dot{\mathrm{v}}_{\mathrm{r}_{\mathrm{gr}}}$ and $\dot{\mathrm{v}}_{\mathrm{rr}}{ }_{\mathrm{gr}}$ were in no way dependent upon the radial wind.

That there may be a few inherent data and computational shortcomings in certain of the individual levels is not denied. The area integrations did require qualitative analysis. It is felt, however, that in the ten-level composites, these possible individual level shortcomings could not and did not disguise the broad-scale features displayed. 
2. Equality of Frictional Acceleration in Fixed and Moving Storm Center (i.e. Frr

The substantial derivative relative to the moving storm center-- i.e.-$\dot{\mathrm{v}}_{\mathbf{r r}}{ }^{--}$is with the previous stated assumption of constant storm translation

$$
v_{\theta r} \frac{\partial v_{r r}}{r \partial \theta}+v_{r r} \frac{\partial v_{r r}}{\partial r}+w \frac{\partial v_{r r}}{\partial z}
$$

Where $v_{\theta r}$ and $v_{r r}$ are the relative tangential and radial wind components. The local derivative $\partial \mathrm{v}_{\mathrm{rr}} / \partial \mathrm{t}$ drops because of the steady state assumption. The relation between the substantial derivative with respect to the moving center and that with respect to the fixed center is then with the statement and footnote of the second paragraph of section 1-b.

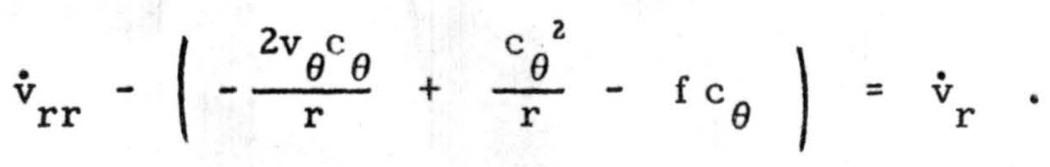

Now

$$
\begin{aligned}
& \left.\dot{v}_{r^{\prime}{ }_{g r}}=\dot{v}_{r}-F_{r}, \text { or } F_{r}=-\left[\dot{v}_{r}\right)_{g r}-\dot{v}_{r}\right] \\
& \left.\dot{v}_{r r}^{\prime}\right)_{g r}=\dot{v}_{r r}-F_{r r}, \text { or } F_{r r}=-\left[\dot{v}_{r r}{ }_{g r}-\dot{v}\right]
\end{aligned}
$$

and

$$
\begin{aligned}
& \dot{\mathrm{v}}_{\mathrm{rr}{ }^{\prime} g r}=\dot{\mathrm{v}}_{\mathrm{r}_{\mathrm{gr}}^{\prime}}+\left(-\frac{2 \mathrm{v}_{\theta} \mathrm{c}_{\theta}}{\mathrm{r}}+\frac{\mathrm{c}_{\theta}^{2}}{\mathrm{r}}-\mathrm{f} \mathrm{c}_{\theta}\right) \\
& \left.\dot{\mathrm{v}}_{\mathrm{rr}}=\dot{\mathrm{v}}_{\mathrm{r}}+\mid-\frac{2 \mathrm{v}_{\theta}^{\mathrm{c} \theta}}{\mathrm{r}}+\frac{\mathrm{c}_{\theta}^{2}}{\mathrm{r}}-\mathrm{fc}_{\theta}\right)
\end{aligned}
$$

from previous stated principles and assumptions. Substituting equation $c$ and $d$ into equation 6

$$
\left.\mathrm{F}_{\mathrm{rr}}=-\left(\dot{\mathrm{v}}_{\mathrm{r}}\right)_{\mathrm{gr}}-\dot{\mathrm{v}}_{\mathrm{r}}\right)=\mathrm{F}_{\mathrm{r}}
$$

The frictional acceleration with respect to the moving storm center is thus shown to be identical with that with respect to the fixed instantaneous center. 


\section{REFERENCES}

(1) Ausman, M., 1959; 'Some Computations of the Inflow Angle in Hurricanes Near the Ocean Surface,' U. of Chicago Project Report, Unpublished Manuscript. 22 pp.

(2) Brunt, D., 1939: Physical and Dynamical Meteorology. Cambridge: The University Press, $428 \mathrm{pp}$.

(3) Haurwitz, B., 1941: Dynamic Meteorology, McGraw-Hill Book Company, Inc. pp 192-195.

(4) Hillary, D. T., and F. E. Christensen, 1957: 'Instrumentation of Nat. Hur. Res. Proj. Aircraft.' Nat. Hur. Res. Proj. Report No. 11. U.S. Weather Bureau.

(5) Hubert, Lester F., 1959: 'Distribution of Surface Friction in Hurricanes, ' J. of Meteor. , Vol. 16 pp 393-404.

(6) Huges, L. A., 1952: 'On the Low-level Wind Structure of Tropical Storms.' J. of Meteor., Vol. 9, pp 422-428.

(7) Jordan, C. L., and D. Hurt, and C. Lowrey, 1960: 'The Structure of Hurricane Daisy on 27 August 1958,' J. of Meteor., Vol. 3 pp 337-348.

(8) La Seur, N. E., 1957: 'An Analysis of Some Detailed Data Obtained by Aircraft Reconnaissance of a Hurricane,' Contract Report, Florida State University.

(9) Malkus, J. S., 1958: 'On the Thermal Structure of the Hurricane Core,' Proc. Tech. Conf. on Hurricanes, Am. Met. Soc., pp D31.

1959: 'Recent Developments in Studies of Penetrative Convection and an Application to Hurricane Cumulonimbus Towers,' Tech. Report No.6, Woods Hole Ocean. Instit., Unpublished Mianuscript, pp 34.

(11) - 1960: 'Cloud Patterns in Hurricane Daisy, 1958,' Tech. Report No. 8, Woods Hole Ocean. Instit. , Unpublished Manuscript. pp 63. 
(12) and H. Riehl, 1960: 'On the Dynamics and Energy Transformations in Steady-State Hurricanes, ' Tellus, Vol. 12, pp $1-20$.

(13) Miller, B. I. , 1958: 'Details of Circulation in the High Energy Core of Hurricane Carrie,' Nat. Hur. Res. Proj. Report No. 24 .

(14) Reynolds, Osborne, 1958: 'On the Dynamical Theory of Incompressible Viscous Fluids and the Determination of Criterion, ' Phil. Trans. Roy. Soc. Lond., (A) 186, pp 123-164.

(15) Riehl, H., 1954: Tropical Meteorology, New York, McGraw-Hill, $392 \mathrm{pp}$.

(16) - - 1959: 'On Production of Kinetic Energy from Condensation Heating,' The Rossby Memorial Volume, and the Nat. Hur. Res. Proj. Report No. 22. 25pp.

(17) -...- and R. C. Gentry, 1958, 'Analysis of Tropical Storm Frieda,' Nat. Hur. Res. Proj. Report No. 17, pp 16.

(18) -..... and J. S. Malkus, 1958: 'On the Heat Balance in the Equatorial Trough Zone, ' Contribution to Palmen's 60th Birthday Volume. Geophsica, Helsinki.

(19) Staff Members, Nat. Hur. Res. Proj., 1958: 'Details of Circulation in the High Energy Core of Hurricane Carrie.' Nat. Hur. Res. Report №. 24 .

(20) Sherman, Leon, 1956: 'On the Wind Asymmetry of Hurricanes,' J. of Meteor., Vol. 13, 500-503. 


\section{TABLES}

$$
-39-
$$


TABLE I.

LEVELS TO WHICH RADIAL ACCELERATION COMPUTATIONS WERE MADE .

\begin{tabular}{|c|c|c|c|c|c|c|c|}
\hline$\underset{*}{\text { Storm }}$ & Date & $\begin{array}{c}\text { Pressure } \\
\text { Level } \\
\end{array}$ & $\begin{array}{l}\text { Approx. } \\
\text { Max. Wind at } \\
\text { Flight Level } \\
(\mathrm{kn})\end{array}$ & $\begin{array}{l}\text { Approx. } \\
\text { Central } \\
\text { Pressure } \\
(\mathrm{mb})\end{array}$ & $\begin{array}{c}\text { Central } \\
\text { Pressure } \\
\text { Change } \\
\end{array}$ & $\begin{array}{c}\text { Storm } \\
\text { Direc- } \\
\text { tion } \\
\end{array}$ & $\begin{array}{r}\text { Speed } \\
(\mathrm{kn})\end{array}$ \\
\hline Daisy & 25 Aug '58 & $950 \mathrm{mb} * *$ & 65 & 990 & deepening & 320 & 7 \\
\hline *Daisy & 25 Aug '58 & $830 \mathrm{mb}$ & 60 & 990 & deepening & 320 & 7 \\
\hline *Daisy & 25 Aug '58 & $570 \mathrm{mb}$ & 60 & 990 & deepening & 320 & 7 \\
\hline Daisy & 25 Aug '58 & $237 \mathrm{mb}$ & 45 & 990 & deepening & 320 & 7 \\
\hline *Daisy & 27 Aug '58 & $630 \mathrm{mb}$ & 120 & 940 & $\begin{array}{l}\text { deepening } \\
\text { then steady }\end{array}$ & 030 & 8 \\
\hline *Daisy & 28 Aug '58 & $620 \mathrm{mb}$ & 105 & 945 & $\begin{array}{l}\text { filling } \\
\text { rapidly }\end{array}$ & 010 & 18 \\
\hline * Carrie & 15 Sept '57 & $605 \mathrm{mb}$ & 85 & 965 & steady & 310 & 12 \\
\hline *Carrie & 17 Sept '57 & $690 \mathrm{mb}$ & 95 & 970 & steady & 080 & 13 \\
\hline *Cleo & 18 Aug '58 & $820 \mathrm{mb}$ & 90 & 970 & steady & 015 & 14 \\
\hline * Cleo & 18 Aug '58 & $580 \mathrm{mb}$ & 85 & 970 & steady & 015 & 15 \\
\hline Cleo & 18 Aug '58 & $255 \mathrm{mb}$ & 50 & 970 & steady & 015 & 14 \\
\hline *Helene & 24 Sept '58 & $635 \mathrm{mb}$ & 60 & 995 & deepening & 320 & 10 \\
\hline *Helene & 25 Sept '58 & $810 \mathrm{mb}$ & 80 & 980 & deepening & 310 & 6 \\
\hline Helene & 26 Sept '58 & $265 \mathrm{mb}$ & 75 & 950 & deepening & 300 & 11 \\
\hline
\end{tabular}

* Used in 10-level composite.

** Composite 1600' and 2800'. 
Max. Wind Max. Wind Difference Speed of Column 4 Storm, Date Right Quad. Left Quad. Right-Left Storm X Column 5 and Level (knots)

(knots) Quad.(kn) Twolkn) (kn)

1. Daisy 25 Aug

65

35

30

14

$\approx 2$

$830 \mathrm{mb}$

2. Daisy 25 Aug

60

45

15

14

$\approx 1$

$570 \mathrm{mb}$

3. Daisy 27 Aug 120

85

35

16

$\approx 2$ $630 \mathrm{mb}$

4. Daisy 28 Aug 105

55

50

36

$\approx 1-1 / 2$

$620 \mathrm{mb}$

5. Cleo 18 Aug

90

50

40

28

$\approx 1-1 / 2$

$820 \mathrm{mb}$

6. Cleo 18 Aug

85

45

40

28

$\approx 1-1 / 2$

$570 \mathrm{mb}$

7. Carrie 15 Aug

85

50

35

24

$\approx 1-1 / 2$

$965 \mathrm{mb}$

8. Carrie 17 Aug

95

65

30

26

$\approx 1-1 / 4$

$970 \mathrm{mb}$

9. Helene 24 Sept

60

55

5

20

$\approx 1 / 4$

$635 \mathrm{mb}$

10. Helẹne 25 Sept

80

55

25

12

$\approx 2$

$810 \mathrm{mb}$ 
TABLE III.

COMPUTATIONS OF $\dot{\mathbf{v}}_{\mathbf{r}}{ }_{\mathrm{gr}}$ ALONG EASTERN RADIAL LEG OF DAISY 25 AUGUST-830 MB

\begin{tabular}{|c|c|c|c|c|c|c|c|c|c|c|c|c|c|c|}
\hline 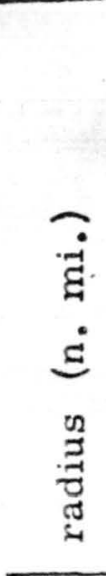 & $\begin{array}{c}s^{N} \mid= \\
\Xi\end{array}$ & 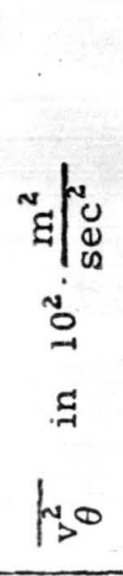 & 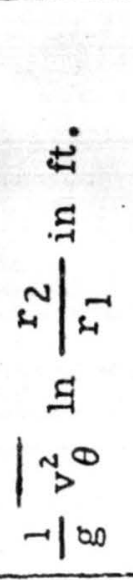 & $\begin{array}{l}q \mid \begin{array}{l}0 \\
0 \\
0\end{array} \\
\mid>^{\circ}\end{array}$ & $\begin{array}{l}\text { من } \\
.7 \\
8 \\
8 \\
4\end{array}$ & $\begin{array}{l}\dot{4} \\
\Xi \\
8 \\
0 \\
1>0 \\
\mid>0\end{array}$ & 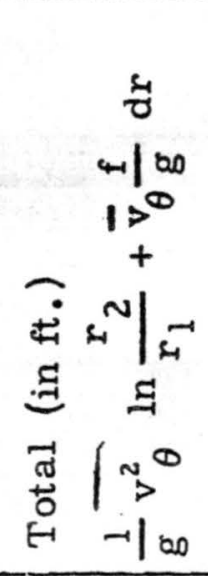 & 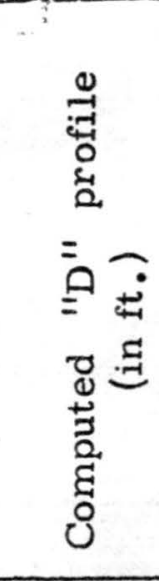 & 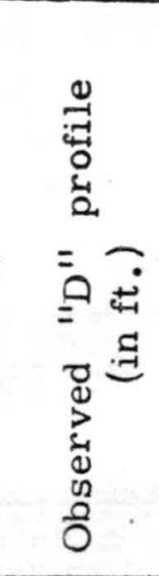 & 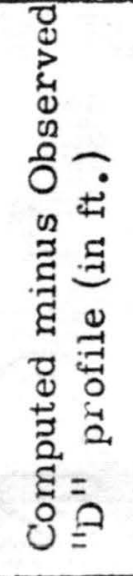 & 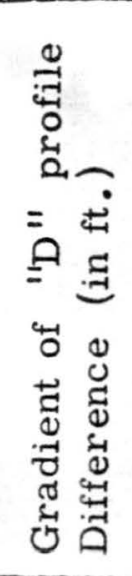 & 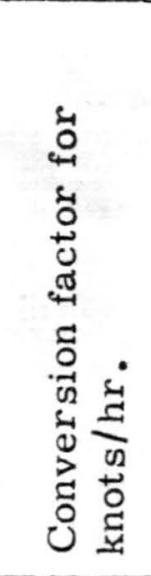 & 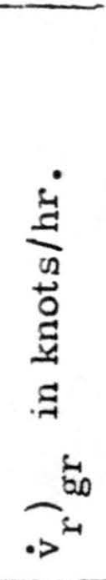 & 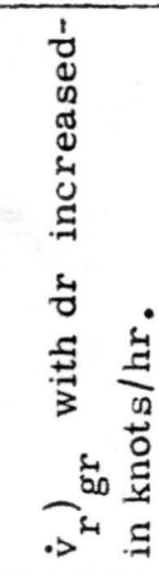 \\
\hline 5 & & & & & & & & -170 & -170 & 0 & & & & \\
\hline & .693 & 4.9 & 111 & 22 & .2 & 4 & 115 & -55 & & & +25 & 2.36 & +59 & +39 \\
\hline 15 & .405 & 8.1 & 107 & 28.5 & .2 & 6 & 113 & $\begin{array}{r}+58 \\
\end{array}$ & & & +8 & 2.36 & +19 & +44 \\
\hline 20 & .288 & 8.1 & 77 & 29 & .2 & 6 & 83 & +141 & +85 & +56 & +23 & 2.36 & +55 & +31 \\
\hline 30 & .405 & 6.6 & 88 & 26 & .4 & 10 & 98 & +239 & +155 & +84 & +26 & 1.18 & +31 & +32 \\
\hline 40 & .288 & 4.5 & 42 & 21.5 & .4 & 8 & 50 & +289 & +200 & $\begin{array}{r} \\
+89\end{array}$ & +5 & 1.18 & +8 & +13 \\
\hline 50 & .223 & 3.3 & 24 & 17.5 & .4 & 7 & 31 & +320 & +230 & -190 & +1 & 1.18 & +1 & +4 \\
\hline 60 & .182 & 3.5 & 21 & 18.5 & .4 & 7 & 28 & +348 & +255 & +93 & +3 & 1.18 & +4 & +7 \\
\hline 70 & .154 & 3.9 & .20 & 20.5 & .4 & 8 & 28 & +376 & 270 & +106 & +13 & 1.18 & +15 & +7 \\
\hline 80 & .134 & 3.4 & 15 & 17.5 & .4 & 7 & 22 & +398 & 290 & -108 & +2 & 1.18 & +2 & +9 \\
\hline
\end{tabular}


DIAGRA M S

$-43-$ 


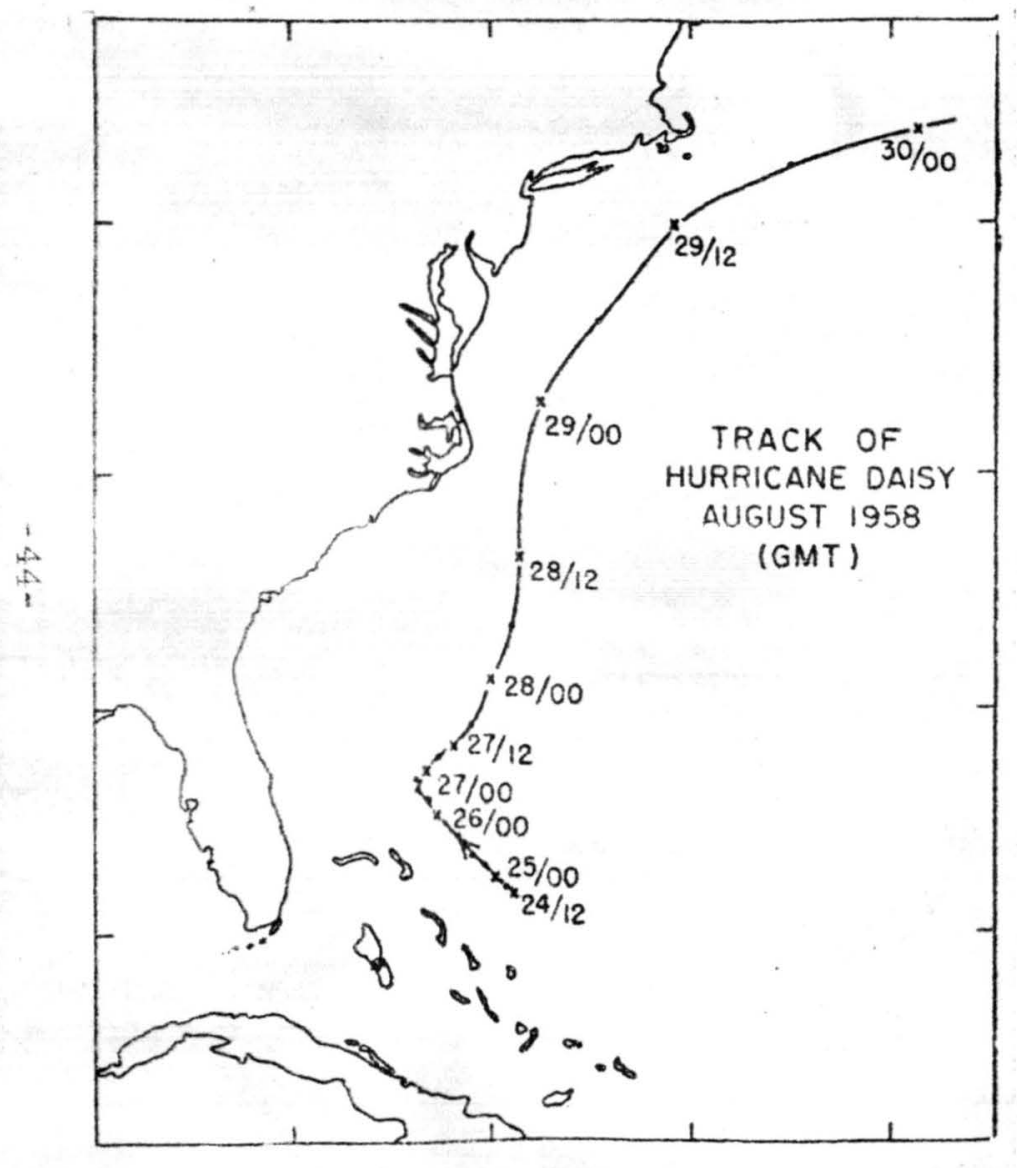

Fig. 1。

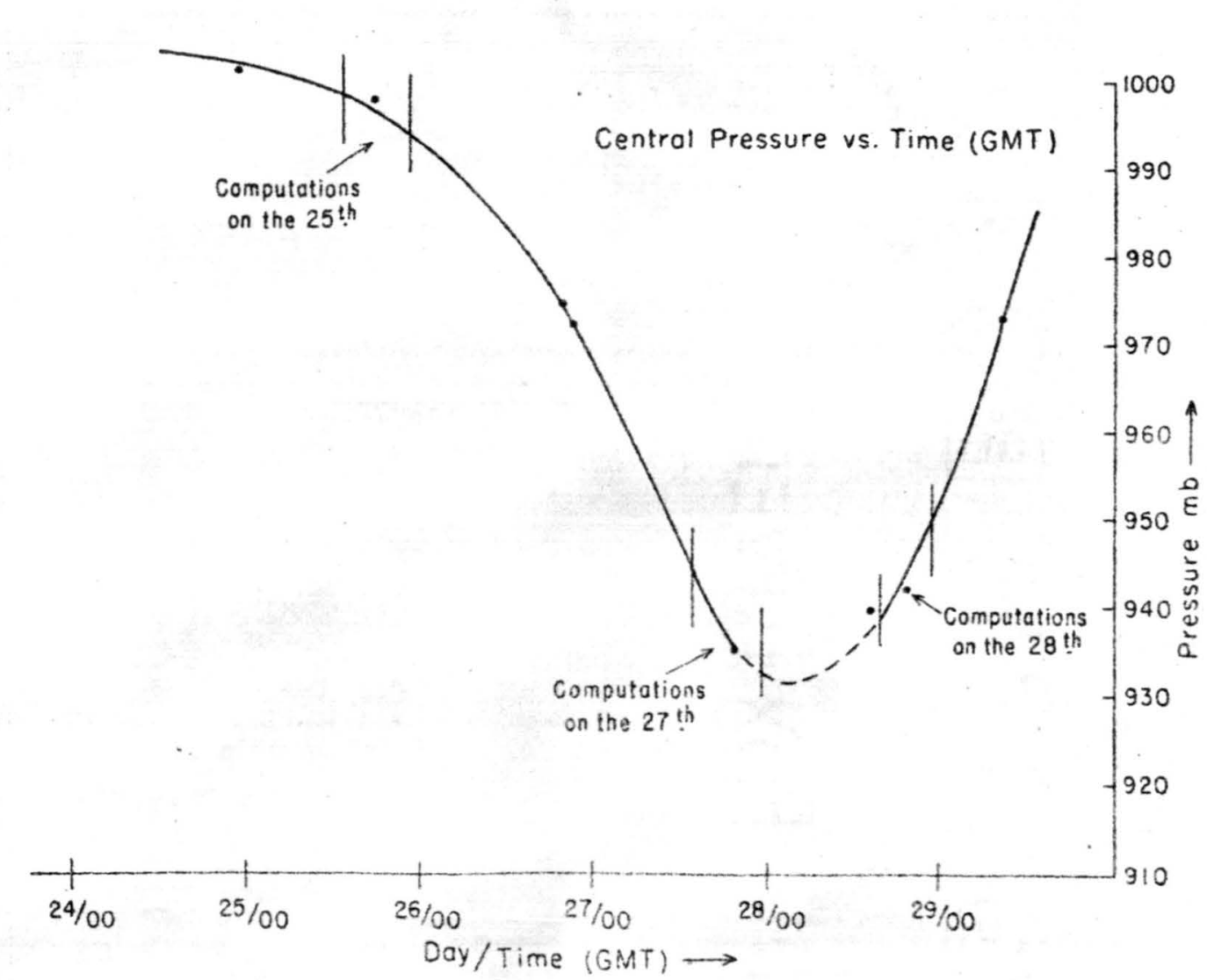

Fig. 2. Central Pressure vs.

Date/time for Hurricane Daisy 


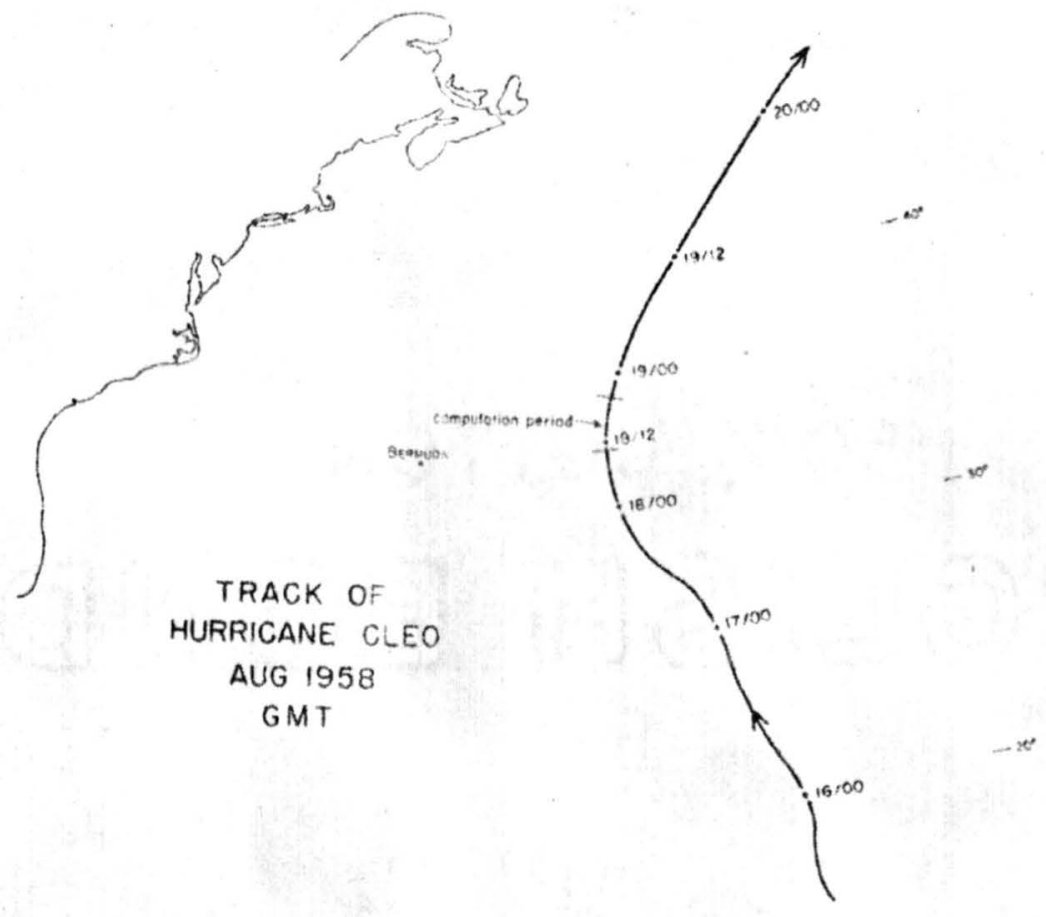

Fig. 3 .
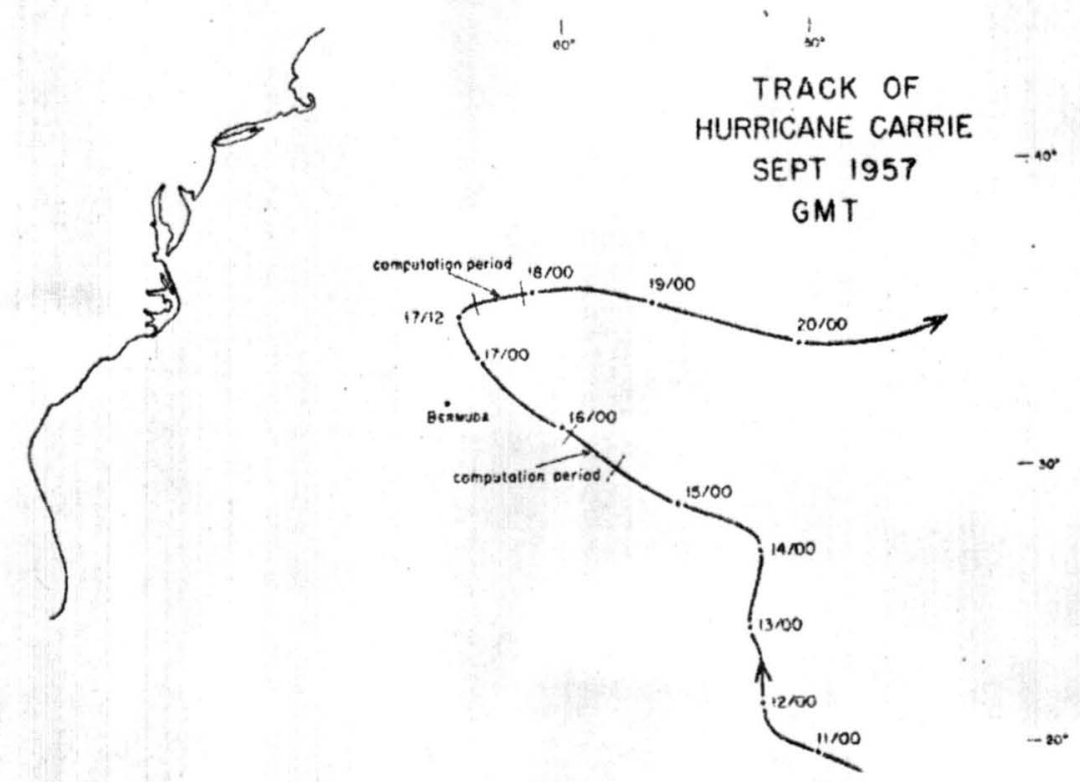

Fig. 4. 


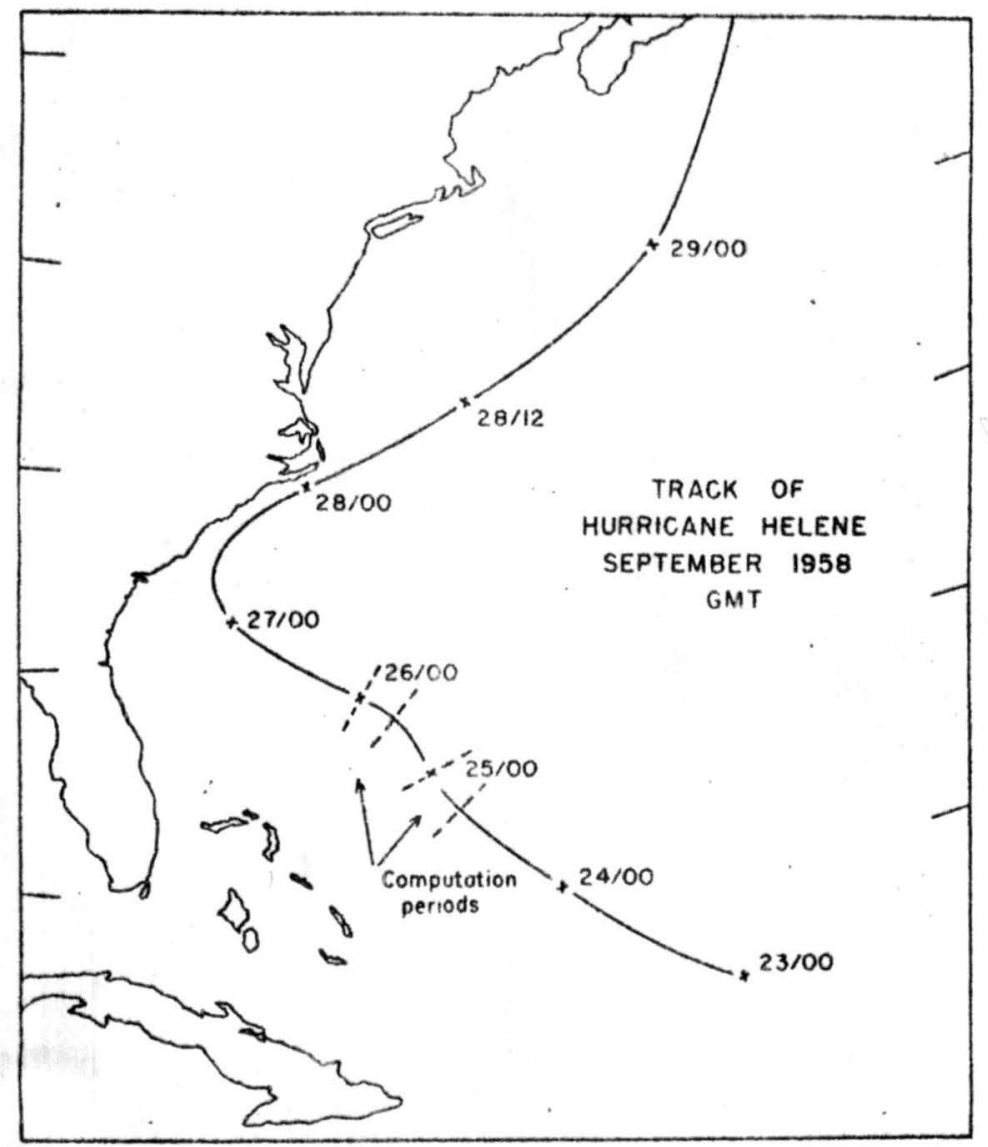

Fig. 5. 


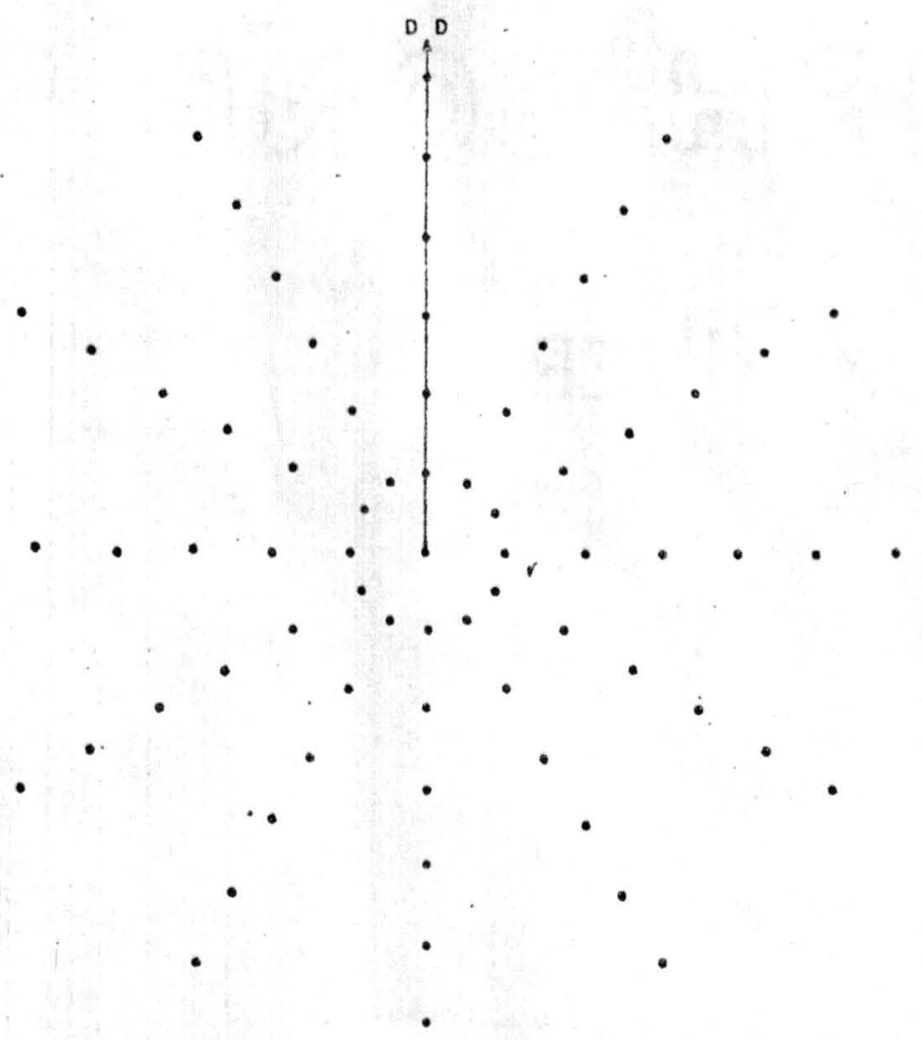

Fig. 6. Grid used in computations 


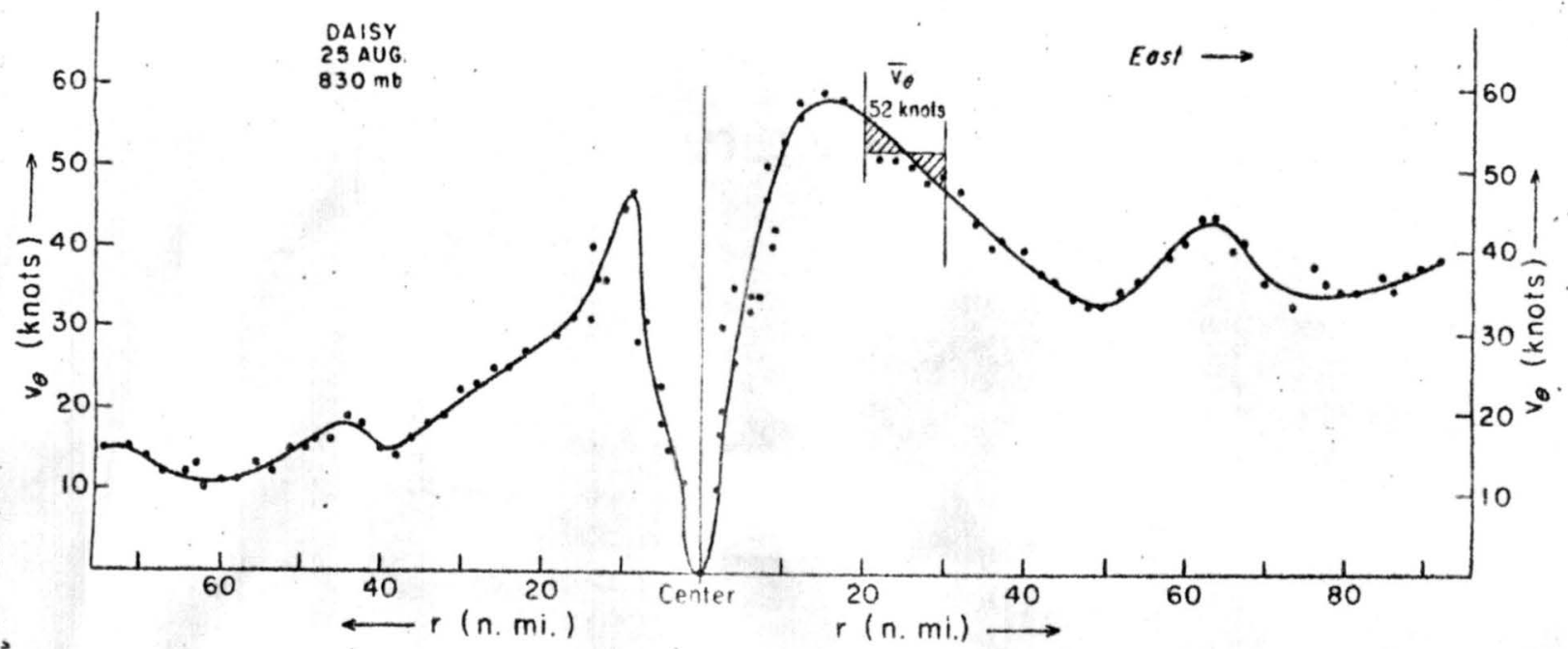

Fig. 7. Profile of the tangential wind $\left(\mathrm{v}_{\theta}\right)$ on a West-East traverse through the center of Daisy. 25 Aug. $830 \mathrm{mb}$.

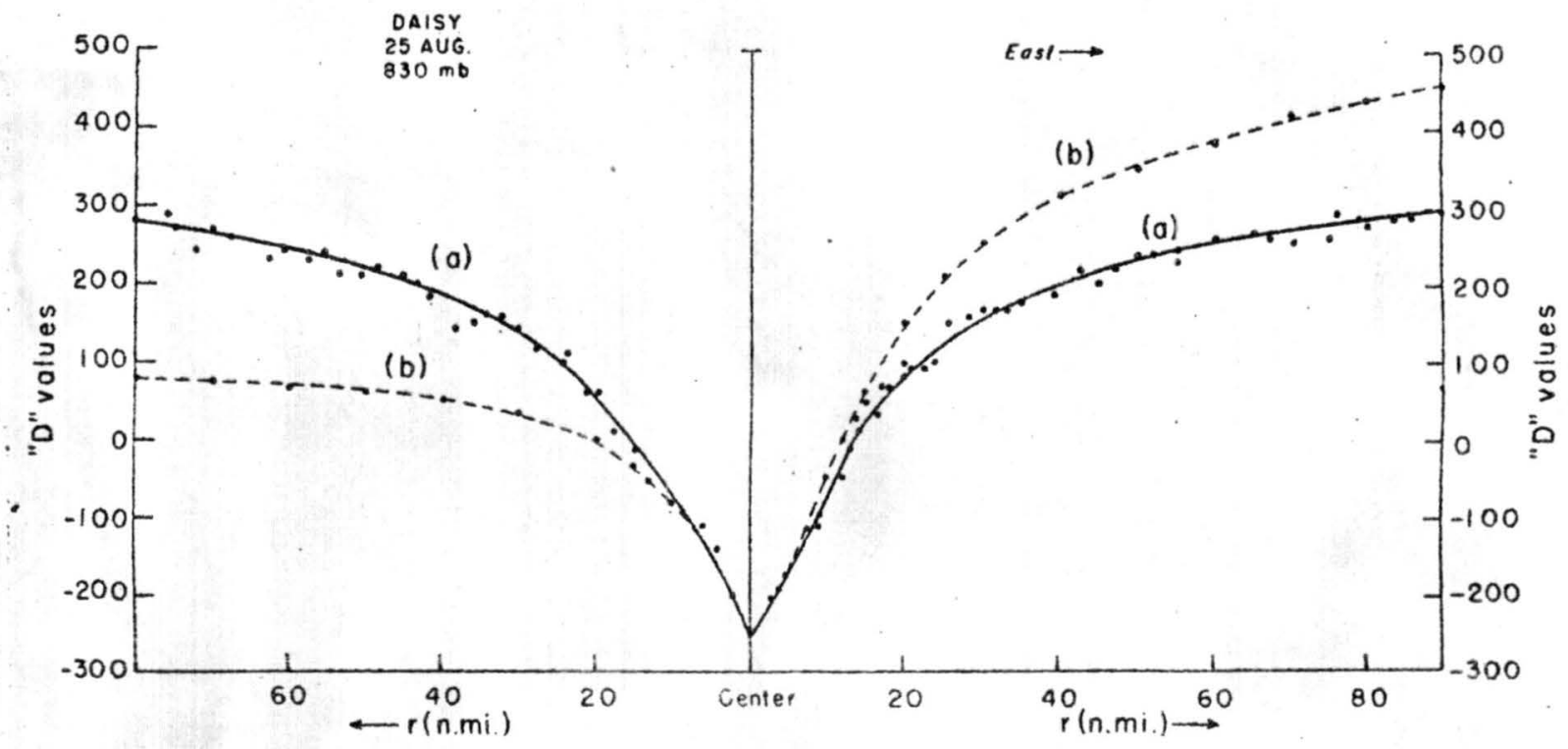

Fig. 8. West-East " $D$ " value profile through the center of Daisy that goes with the $v_{\theta}$ profile in Fig. 3. Curve (a)--Actual "D" profile. Curve (b)--Computed "D" profile (i.e. $\mathrm{D}_{\mathrm{gr}}$ ) 


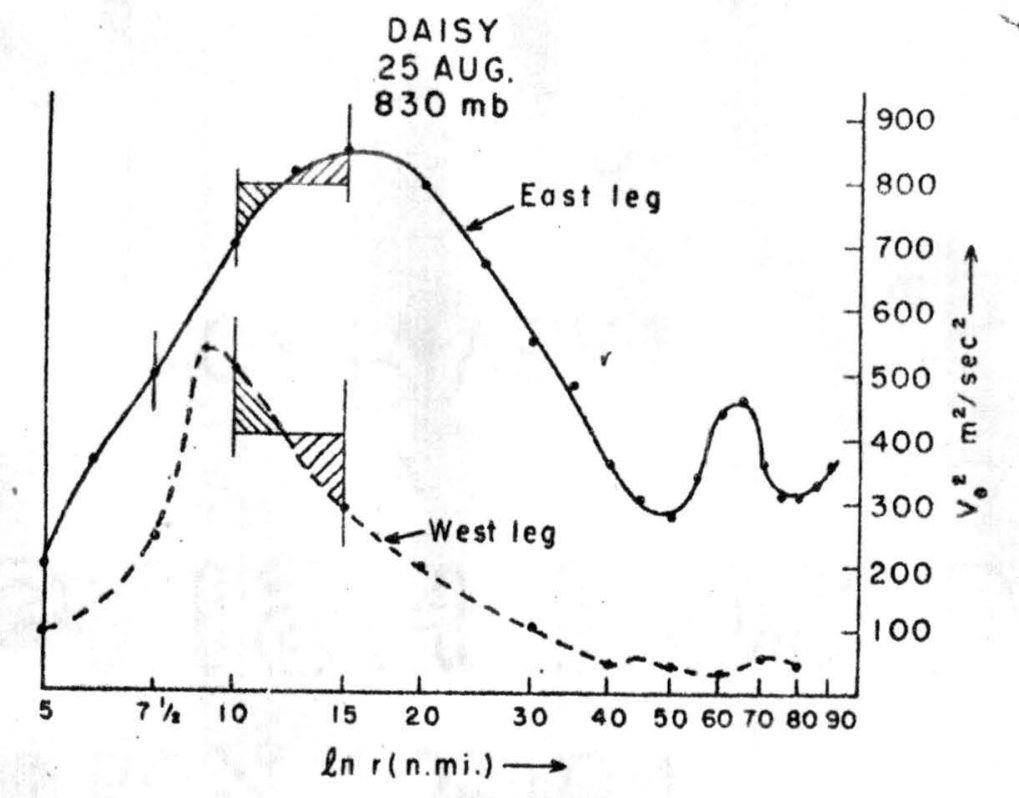

Fig. 9. Comparison of $v_{\theta}^{2}\left(\mathrm{~m}^{2} / \mathrm{sec}^{2}\right)$ profiles along the East and the West legs of the traverse through Daisy on the 25th at $830 \mathrm{mb}$. Values of $\mathrm{v}_{\theta}^{2}$ are plotted aginst in $\mathrm{r}$. 


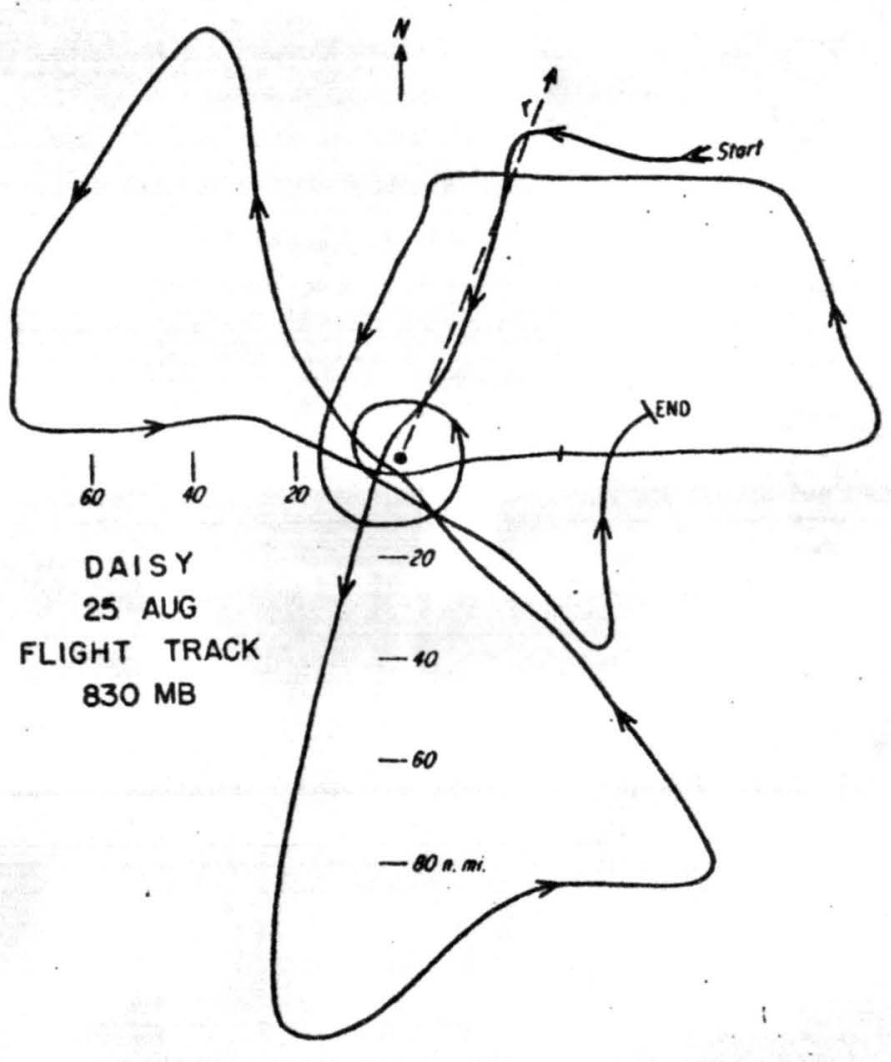

Fig. 10 .
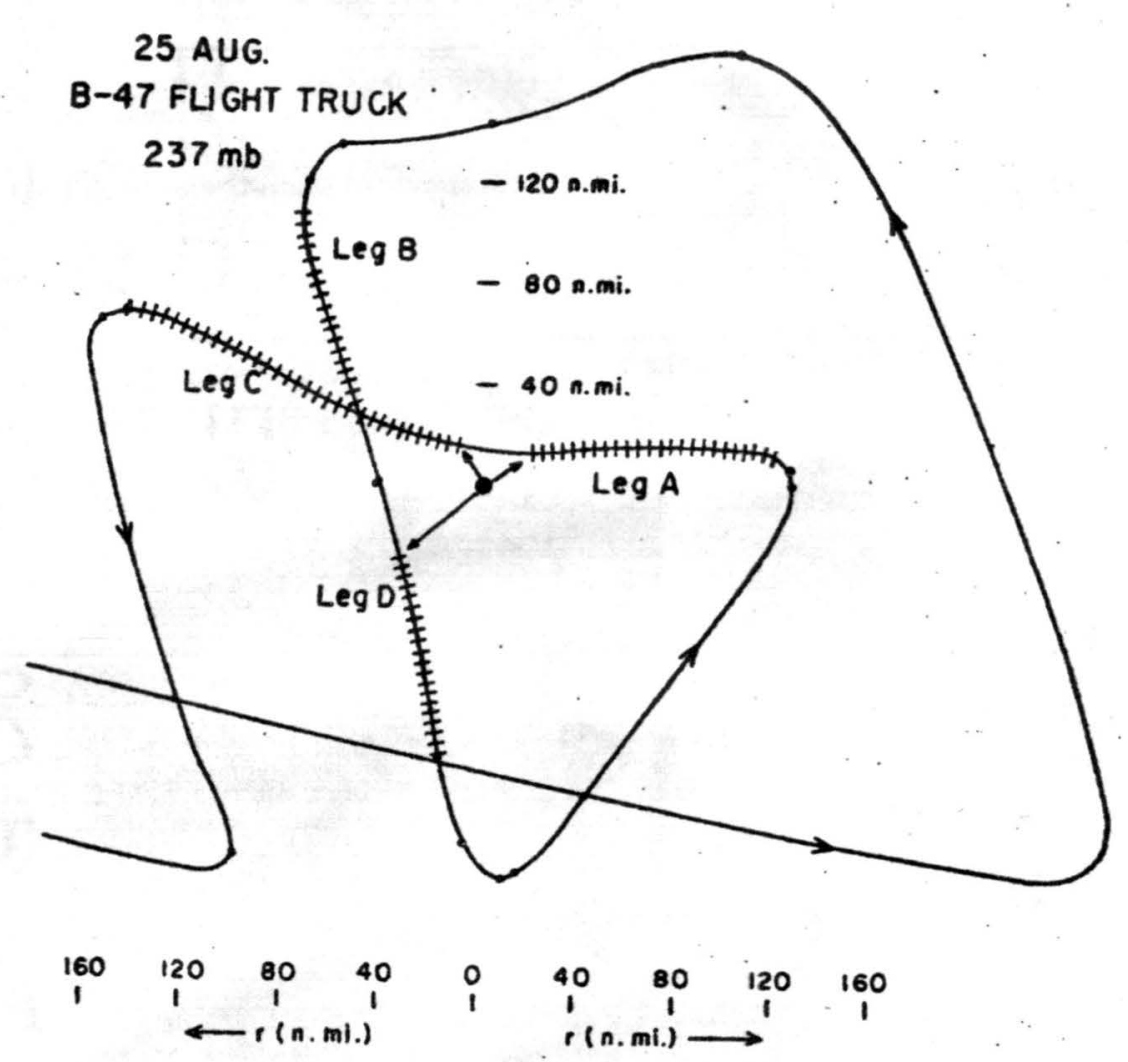

Fig. 11. Track of B-47 on the 25 th at $237 \mathrm{mb}$. Cross-hatching on the track lines indicate places where the $\left.\dot{v}_{\mathbf{r}}\right)_{\text {gr }}$ Computation was performed. 


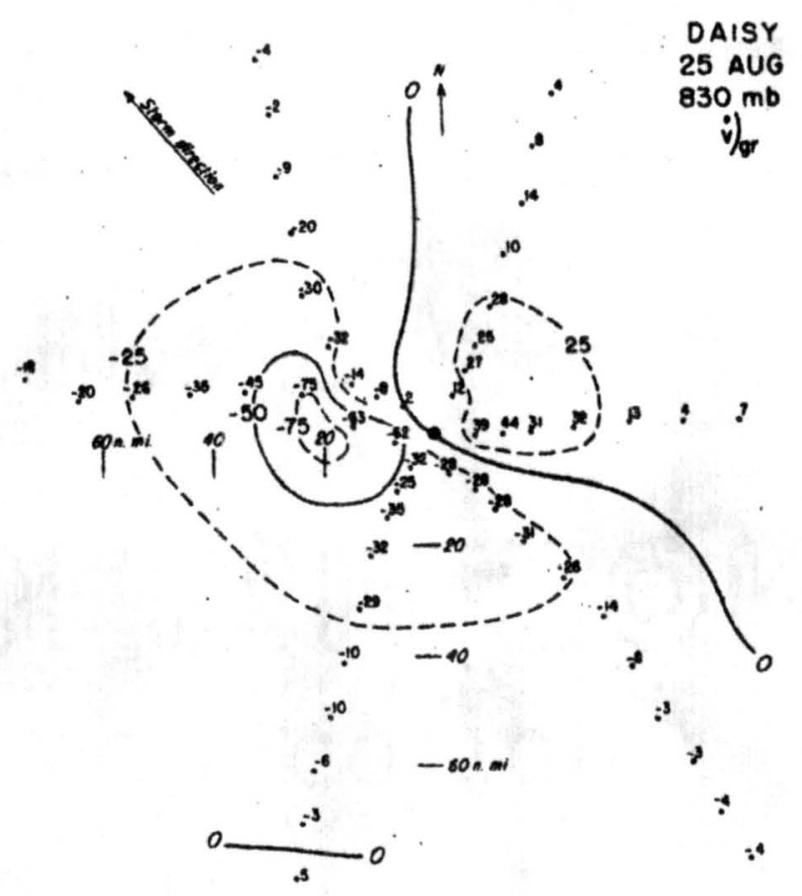

Fig. 12. $\left.\dot{\mathrm{v}}_{\mathbf{r}}\right)_{\mathrm{gr}}$ in knots/hour

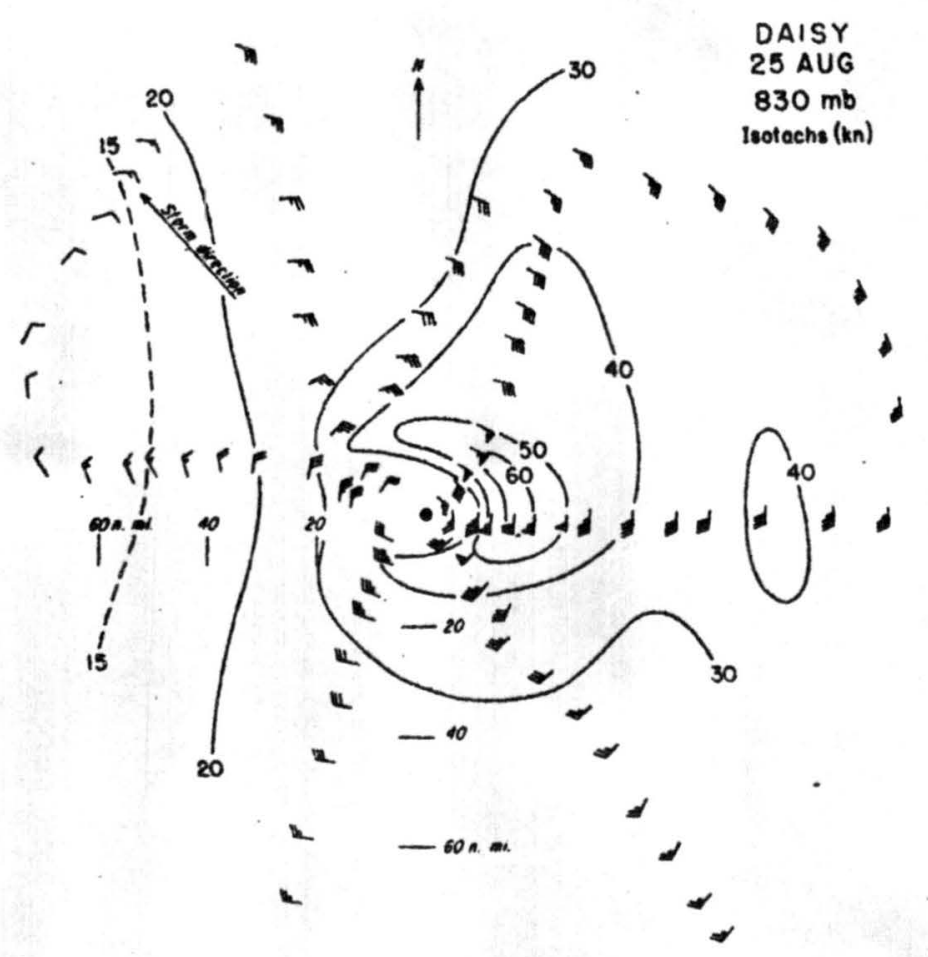

Fig. 13. 


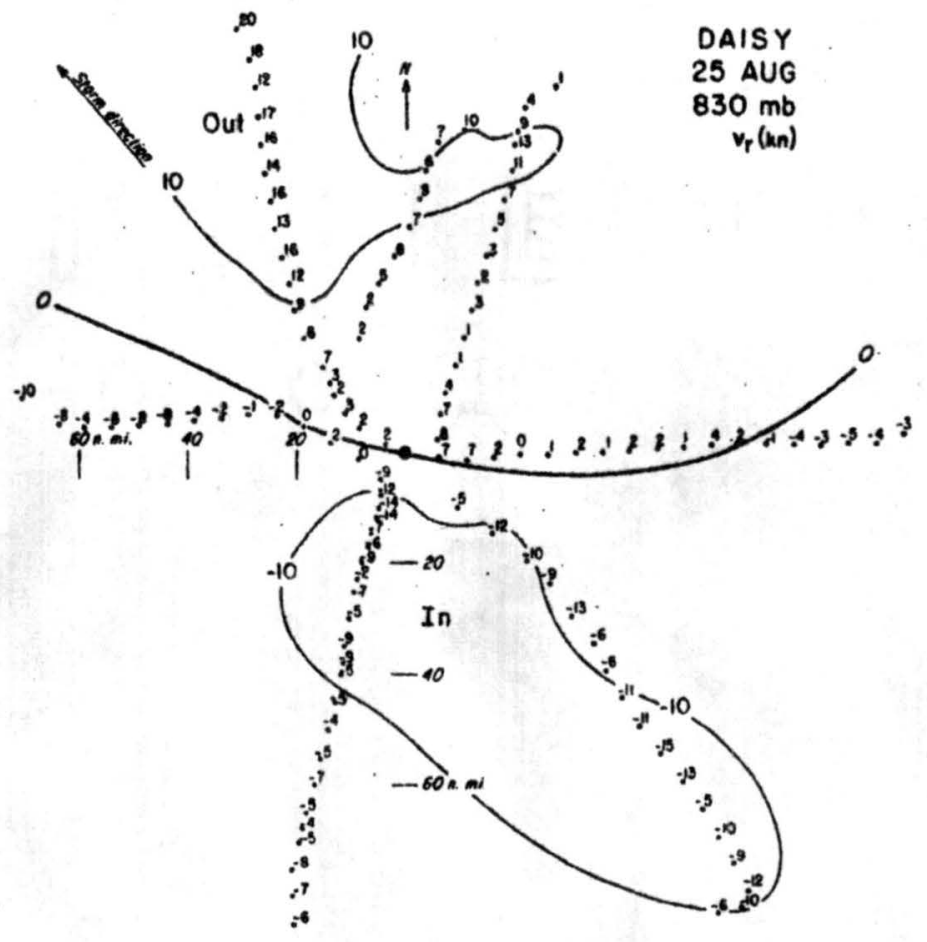

Fig. 14. Radial wind component

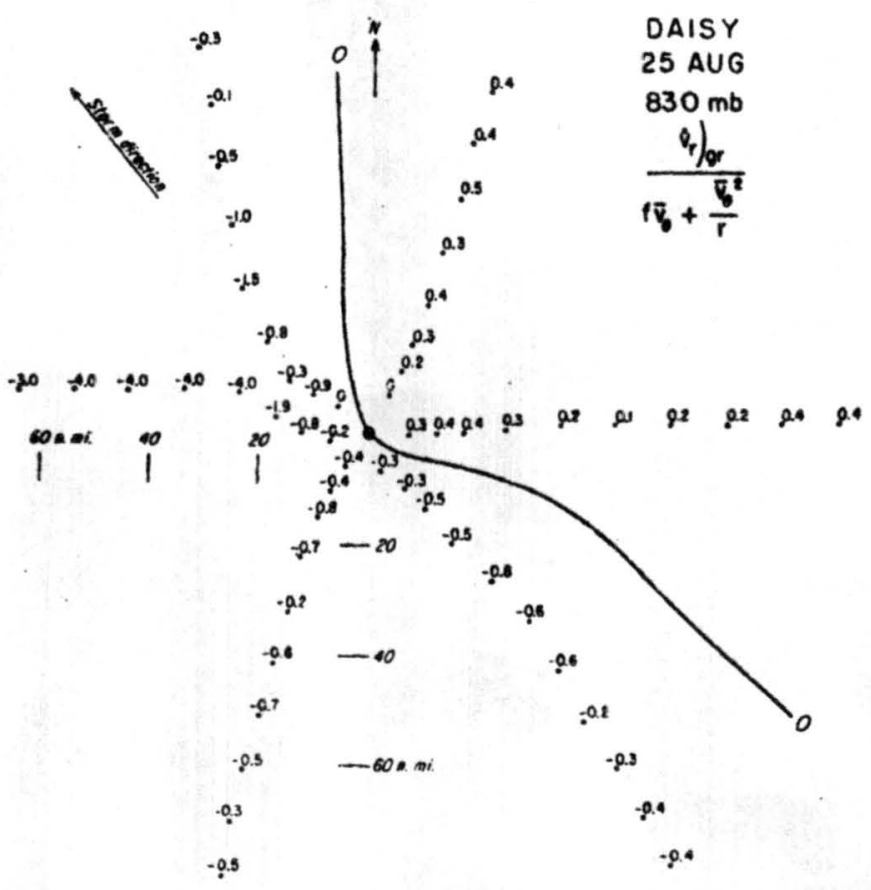

Fig. 15. 


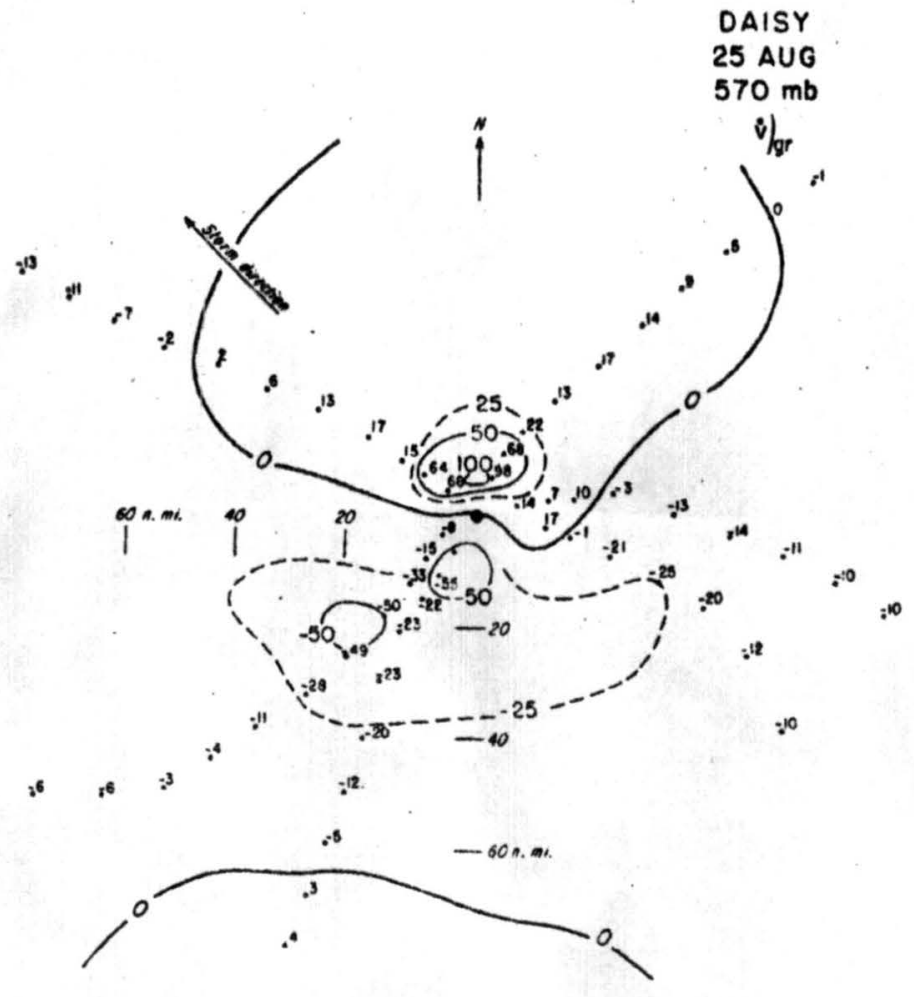

Fig. 16. $\left.\dot{\mathrm{v}}_{\mathrm{r}}\right)_{\mathrm{gr}}$ in knots/hour

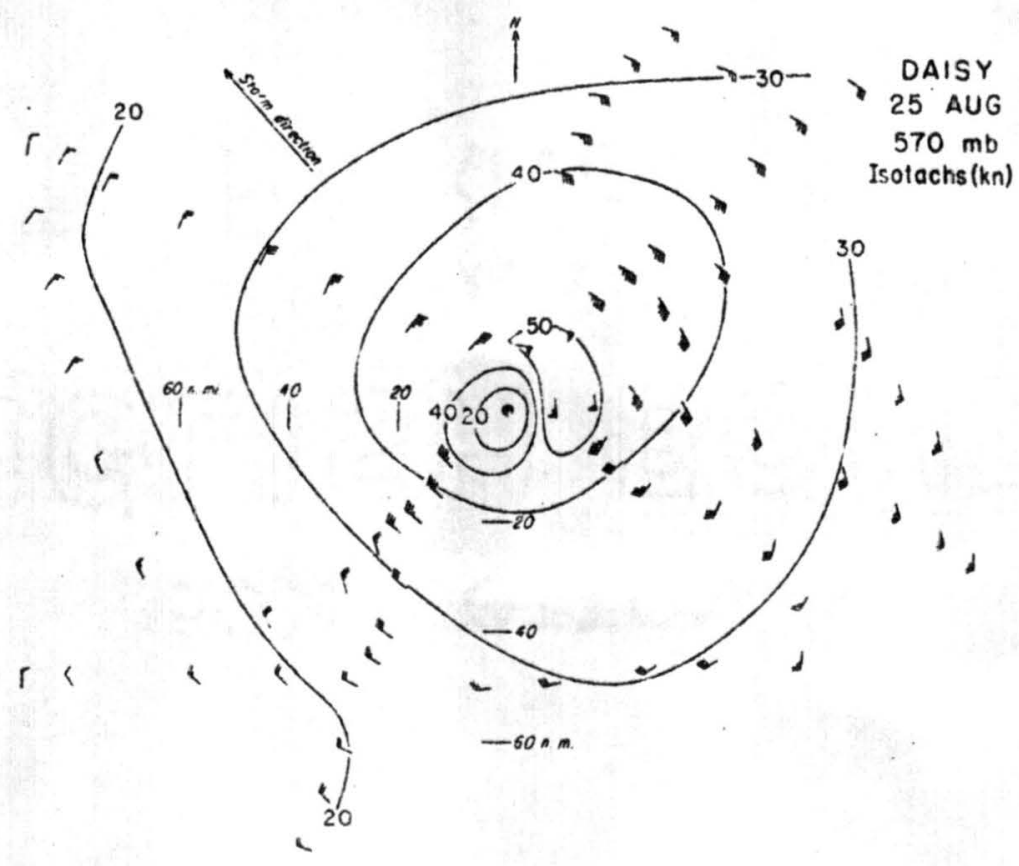

Fig. 17 


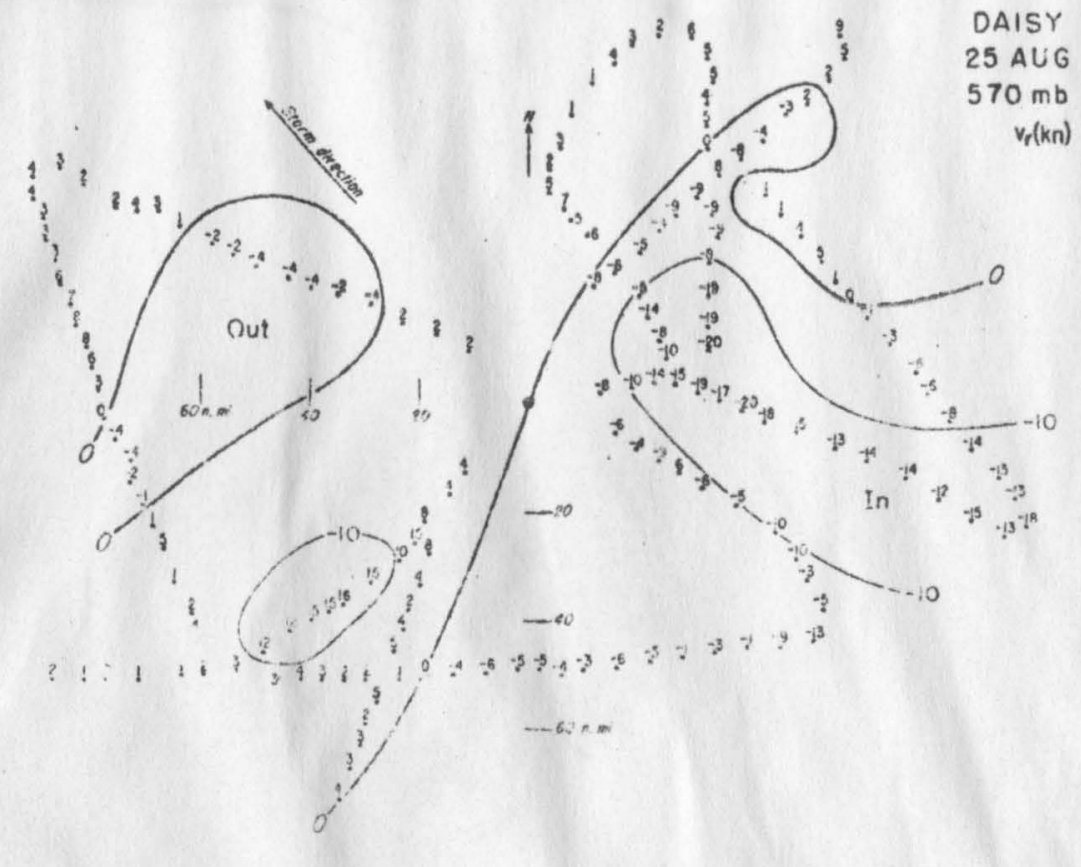

Pig. 13. Radis wina womp ten

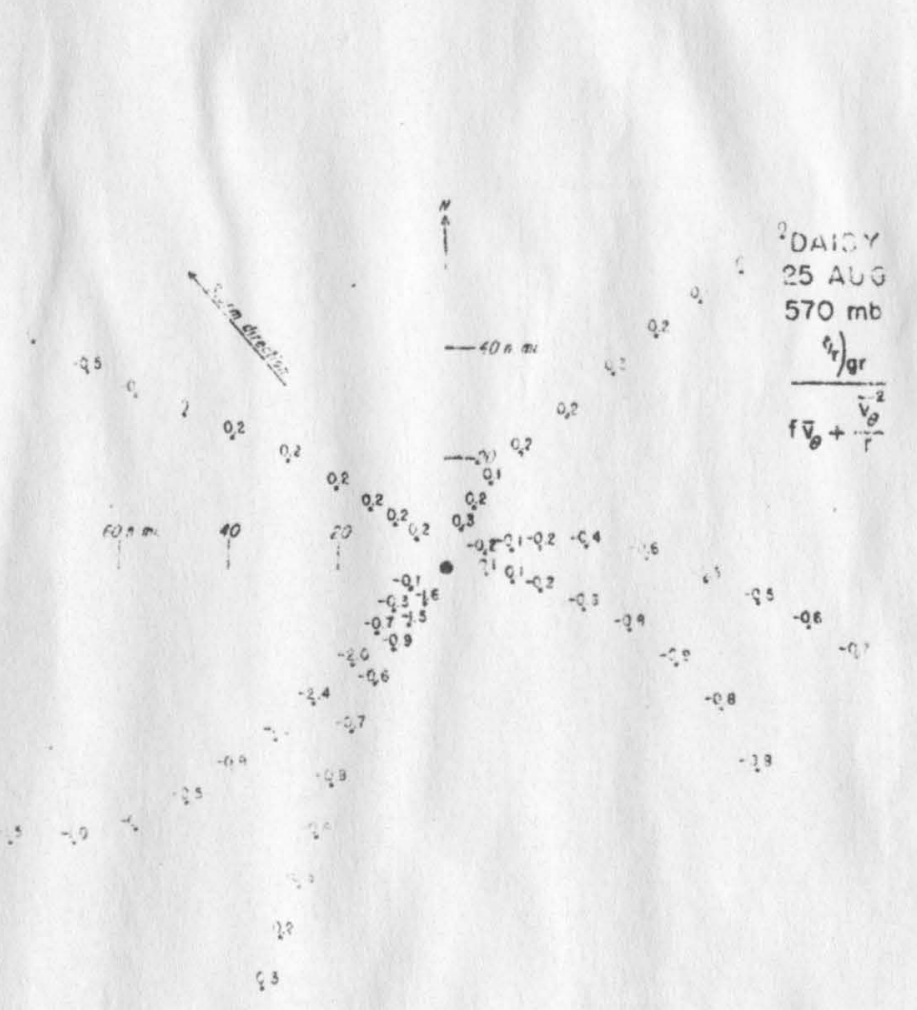

Fis. 19. 


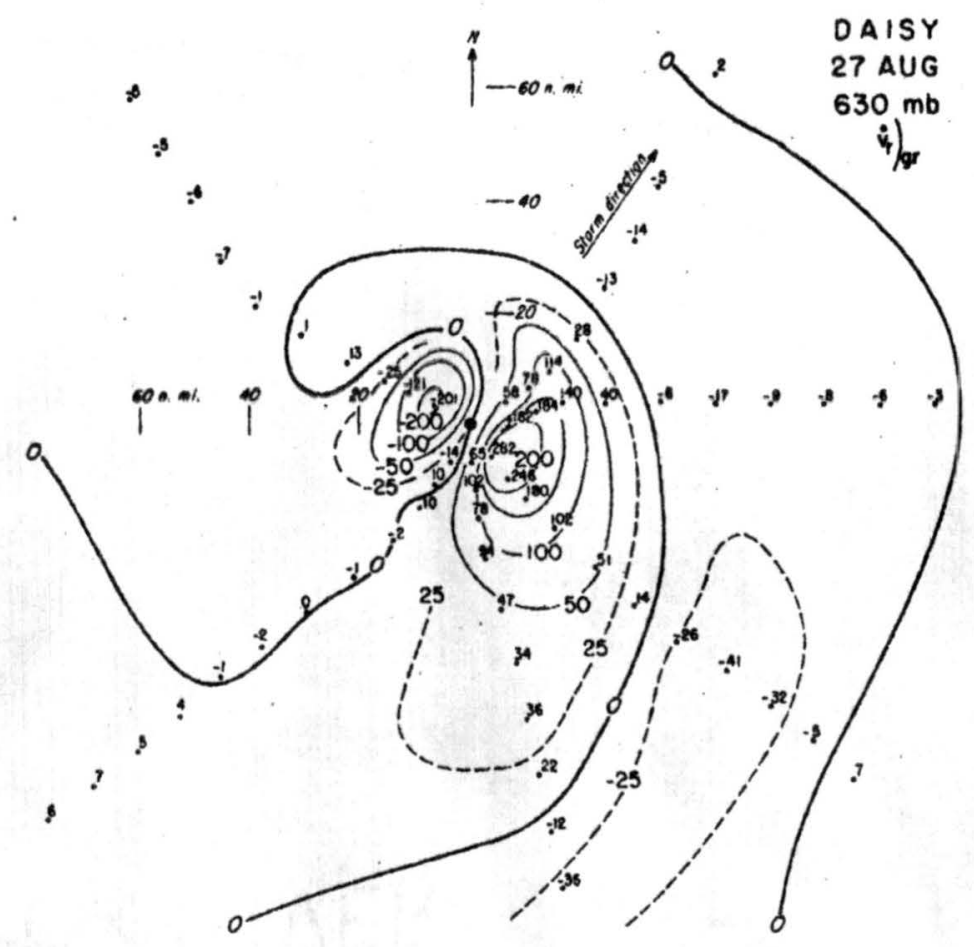

Fig. $20 \quad \dot{\mathrm{v}}_{\mathrm{r}}{ }_{\mathrm{gr}}$ in knots/hour

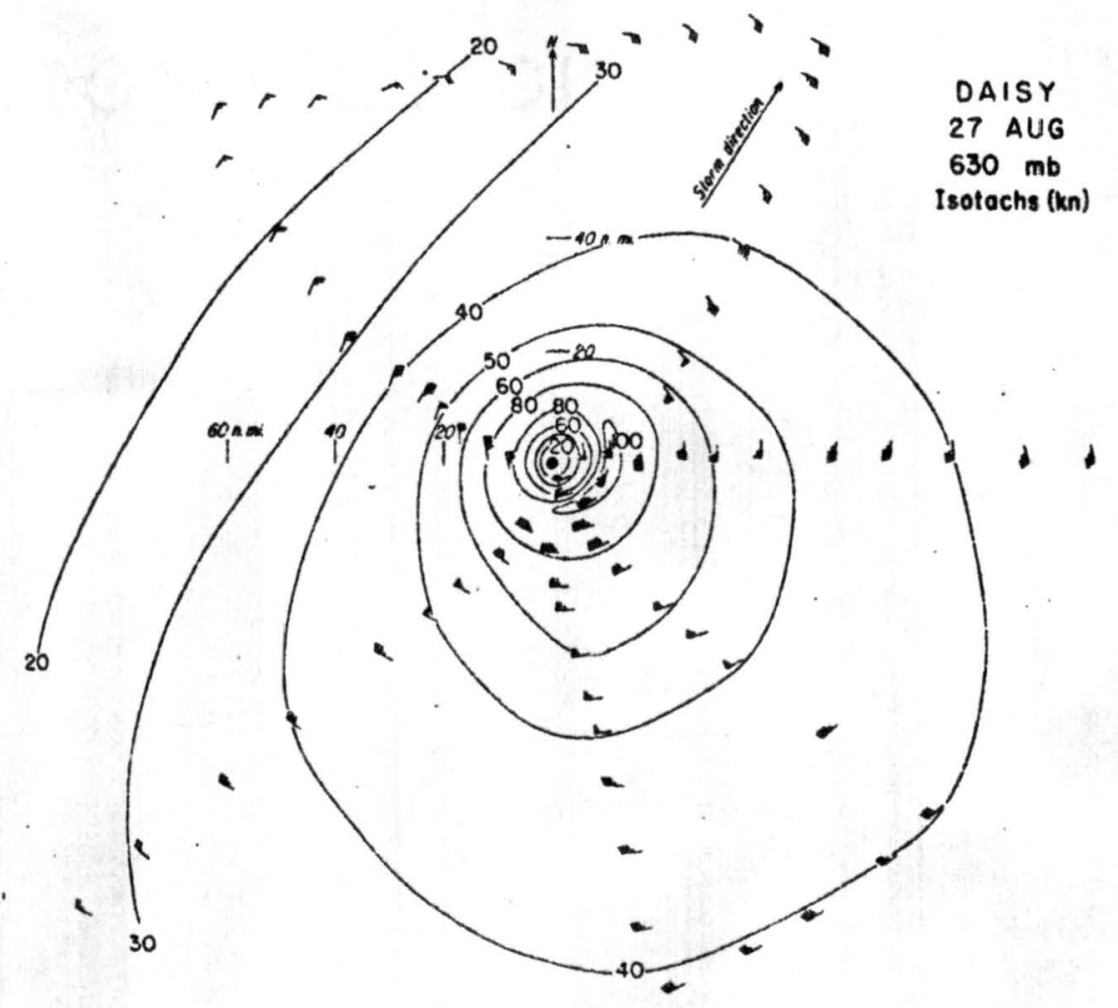

Fig. 21 


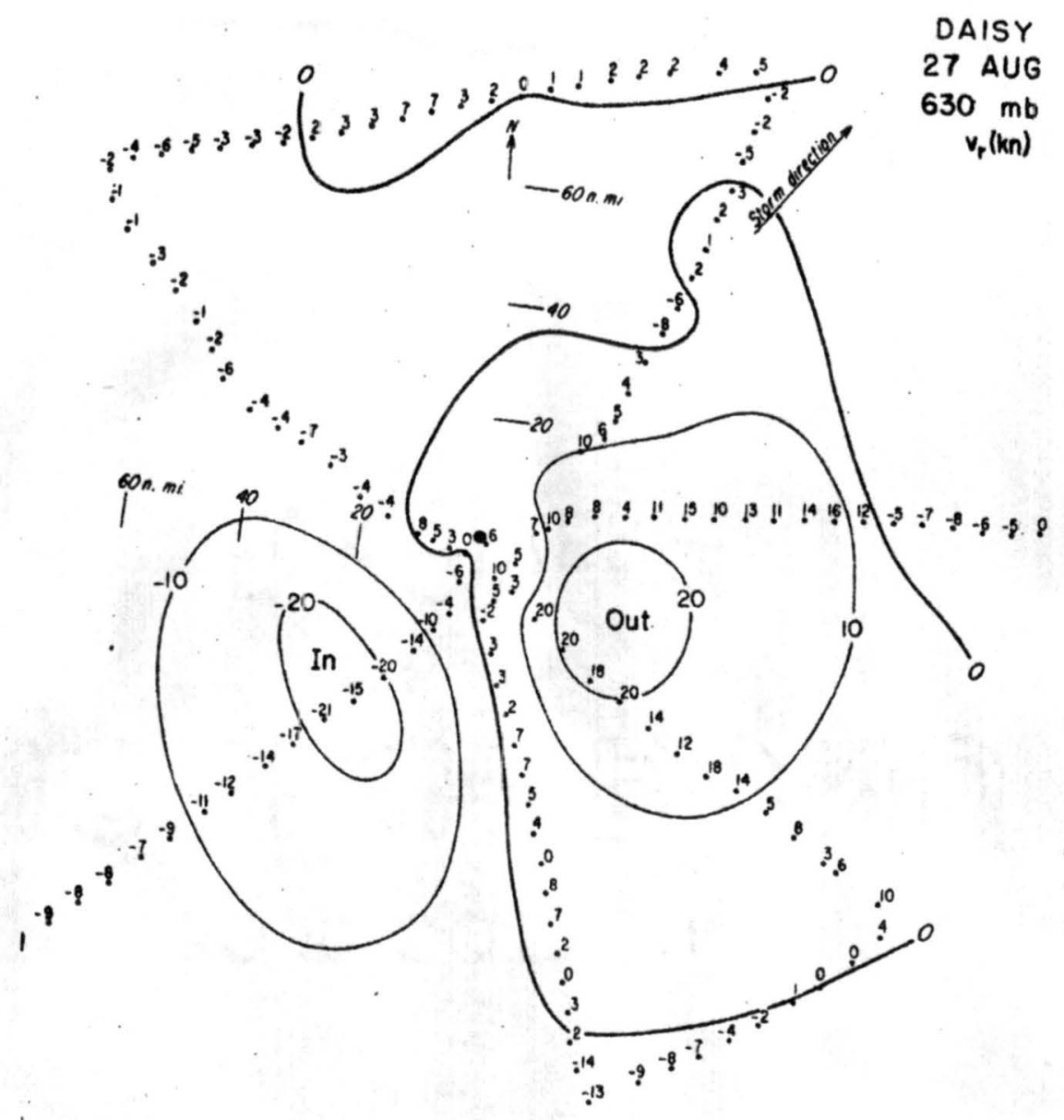

Fig. 22. Radial wind component

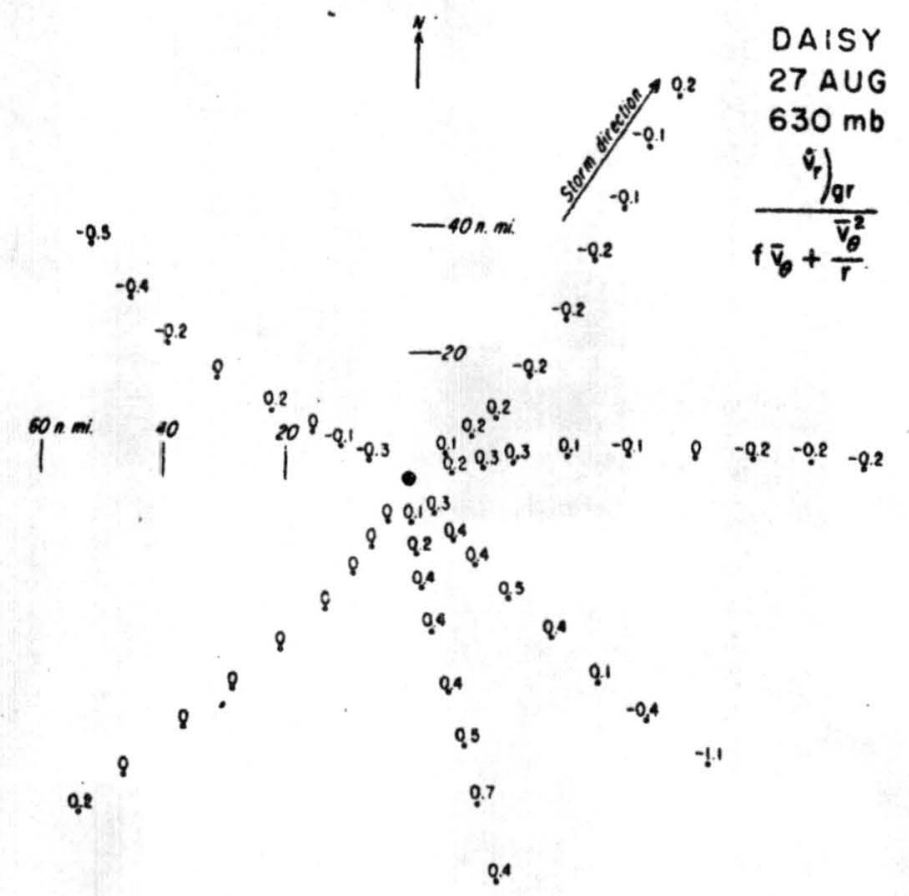

Fig. 23. 


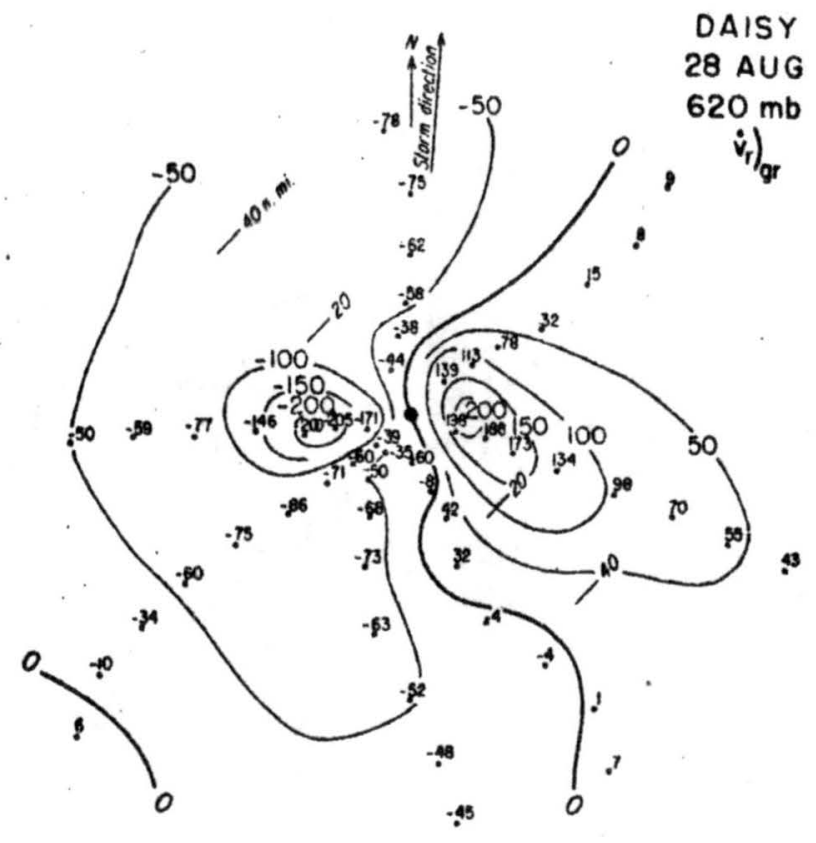

Fig. 24: $\left.\dot{\mathrm{v}}_{\mathrm{r}}\right)_{\mathrm{gr}}$ in knots/hour

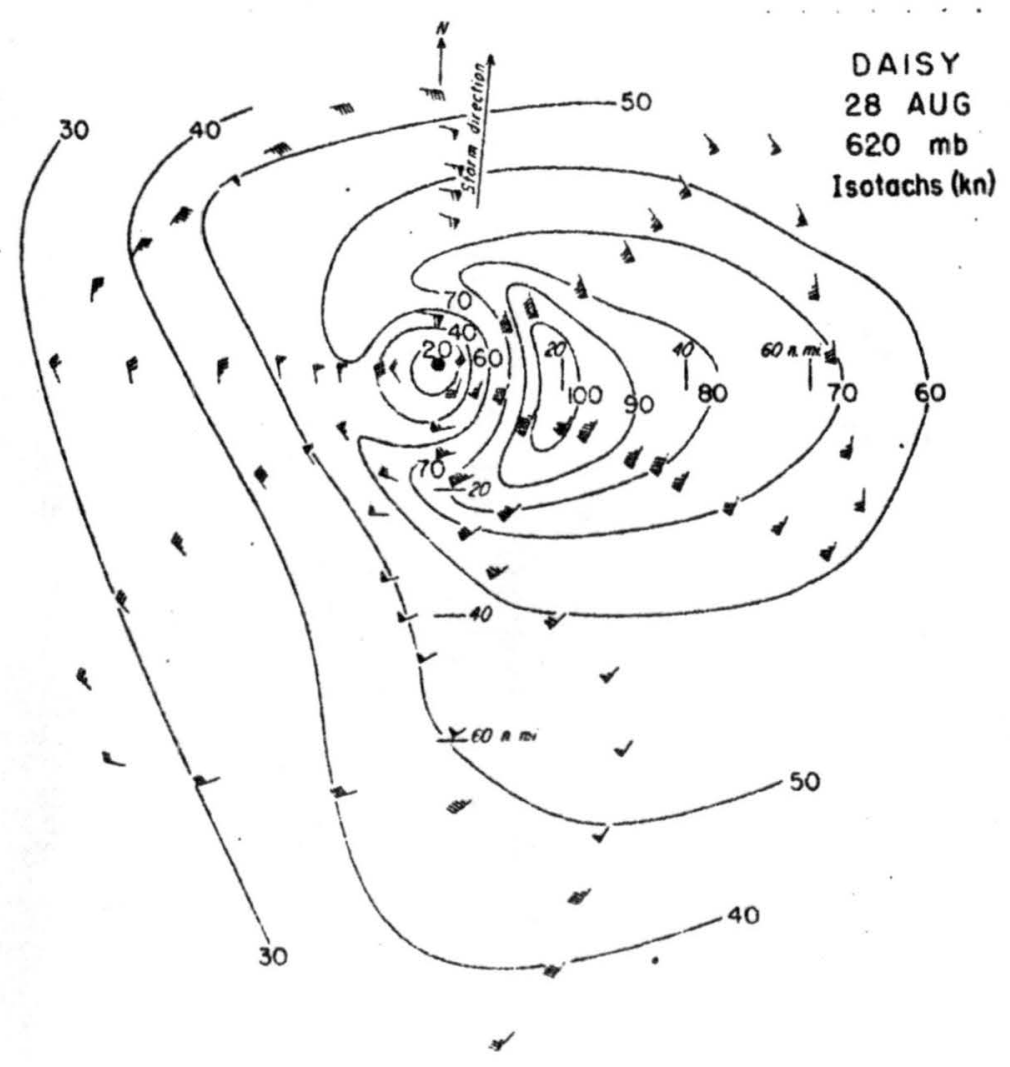

Fig. 25 . 


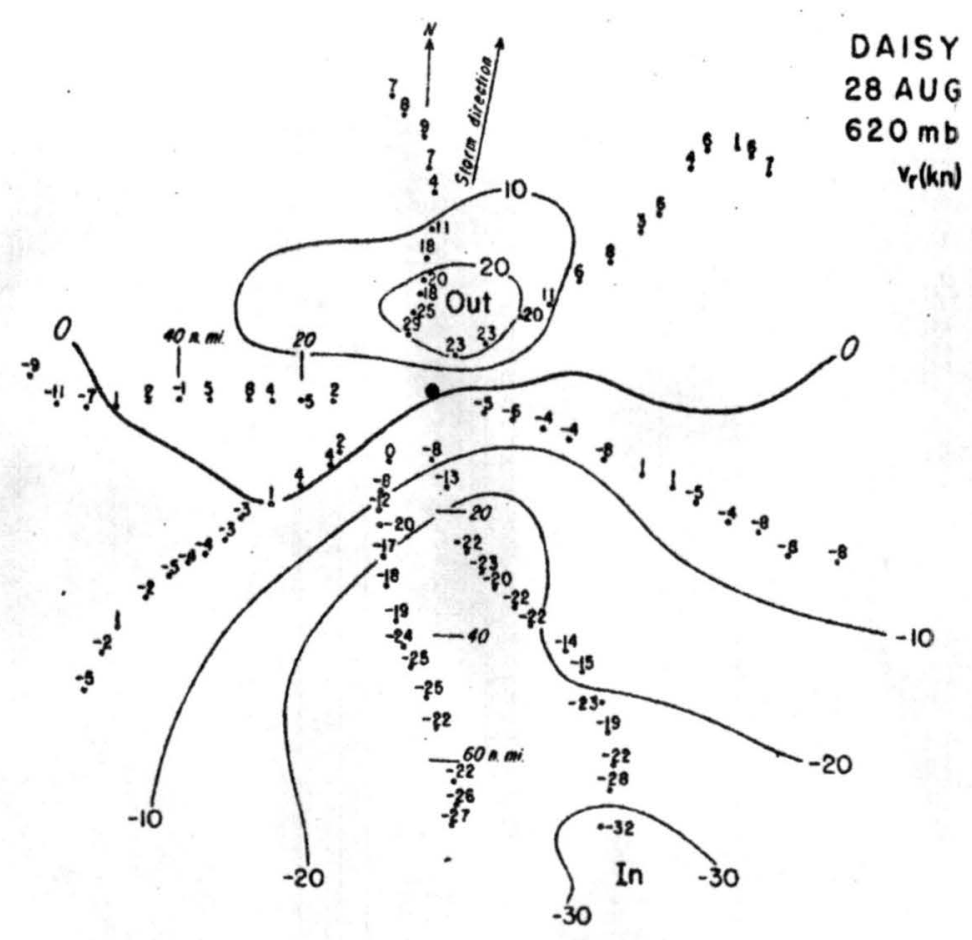

Fig. 26. Radial wind component

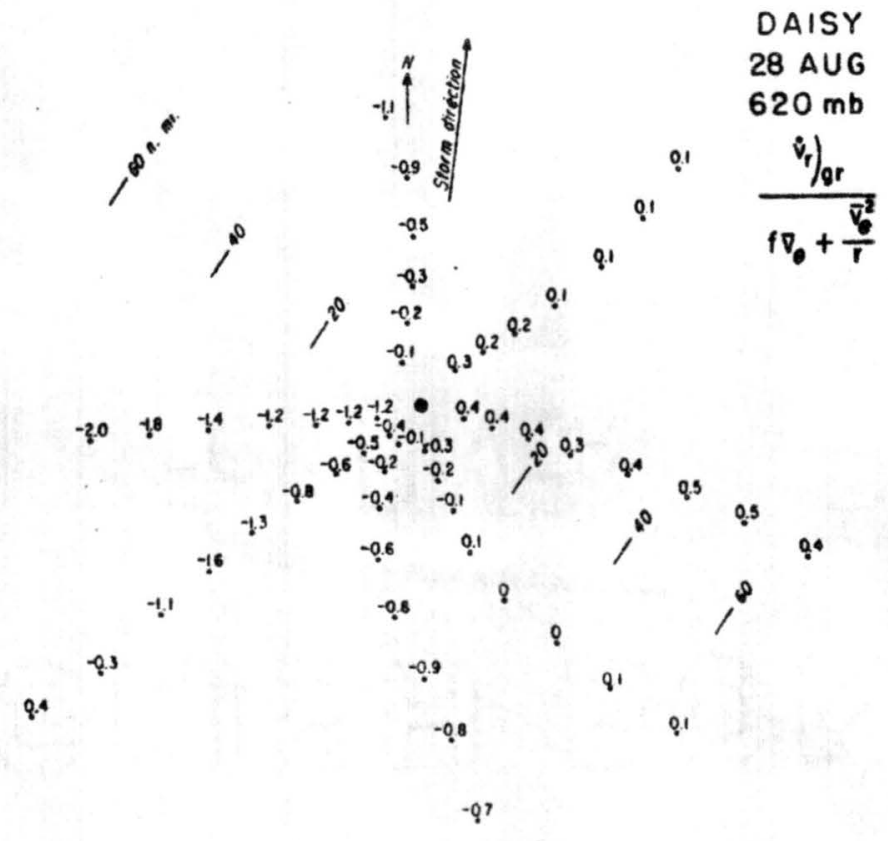

Fig. 27. 


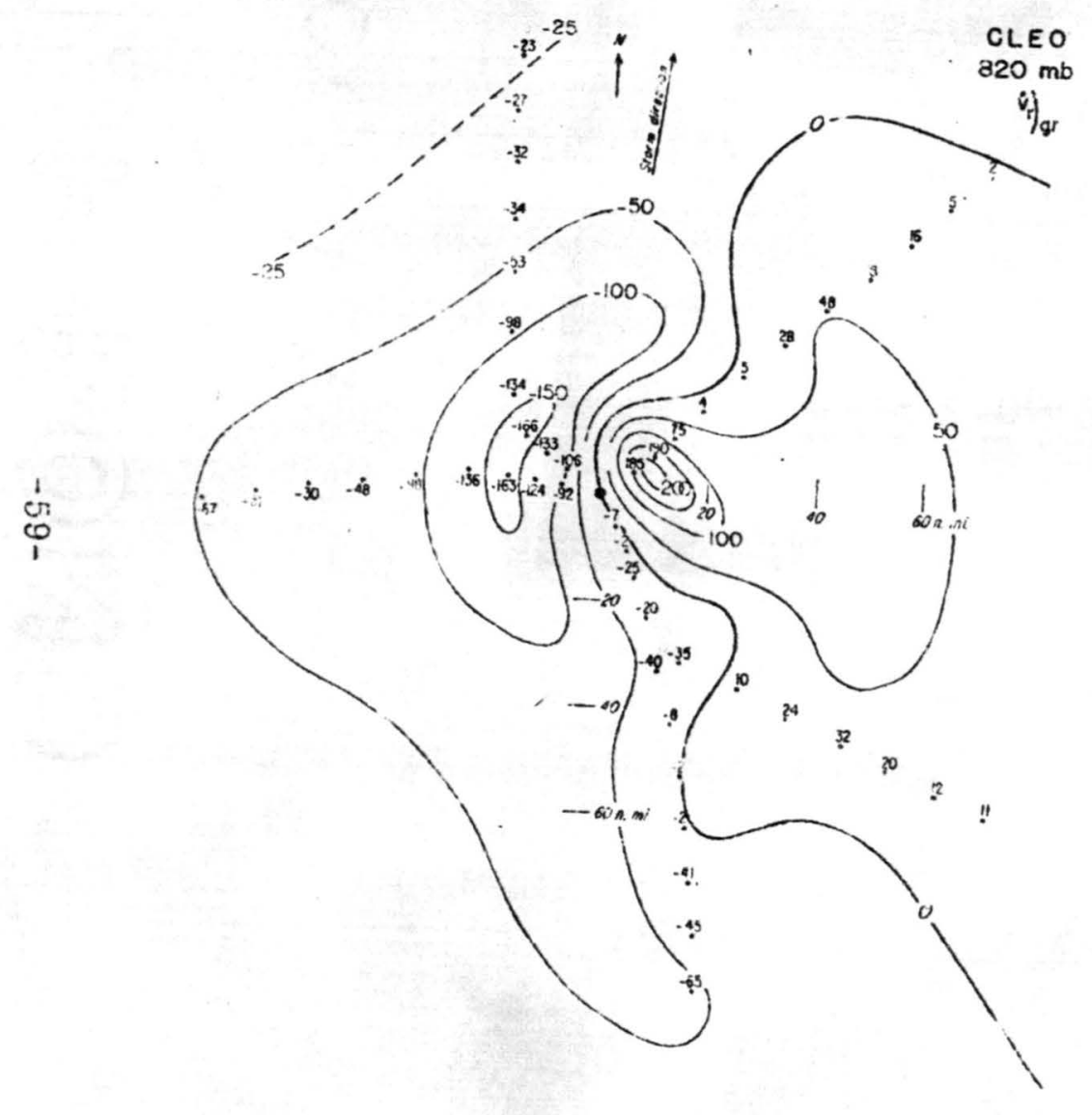

Fig. 28. $\dot{v}_{r_{g r}^{\prime}}$ in knots/hriar

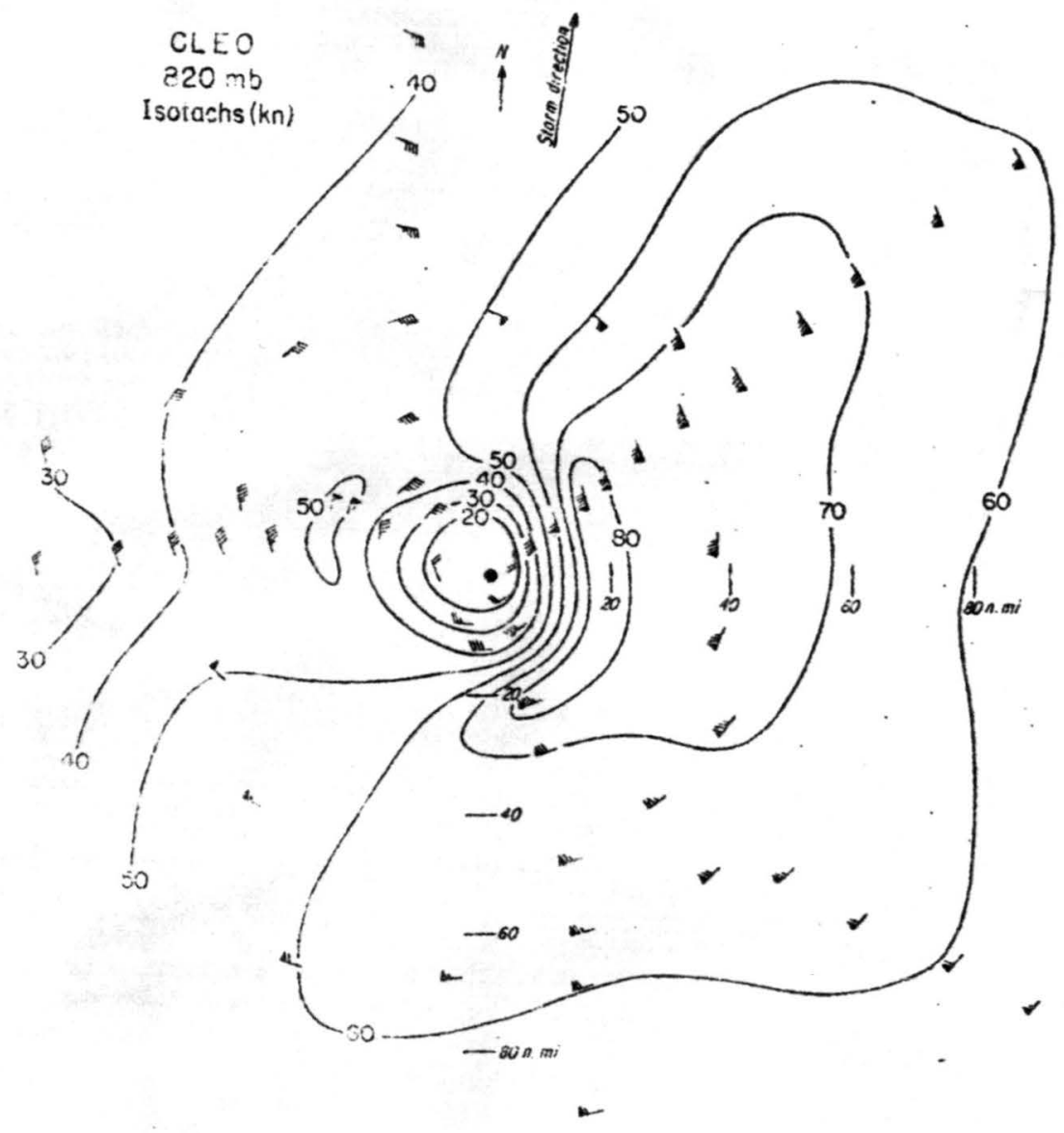

Fig. $2 y$ 


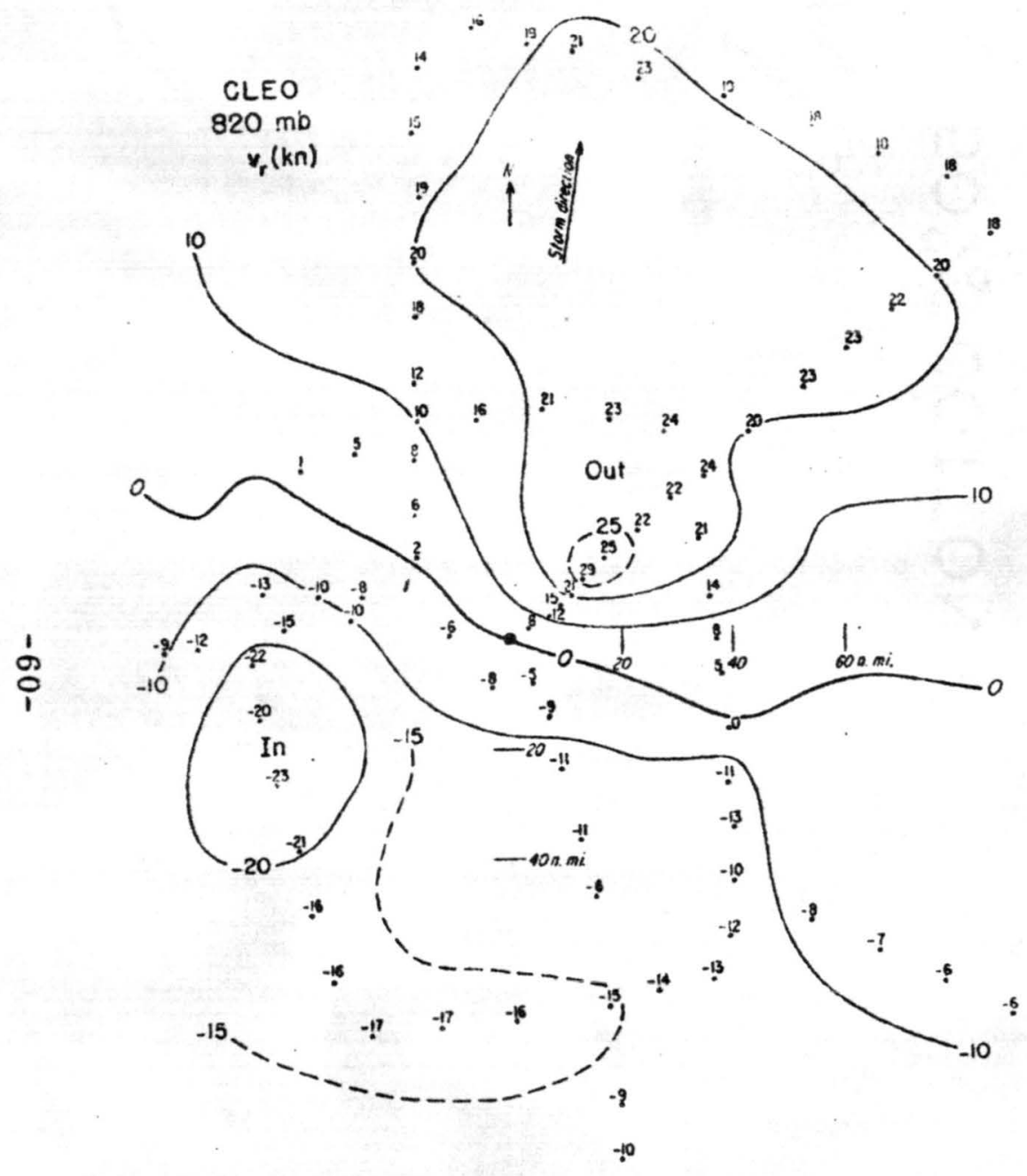

Fig. 30. Radial wind component

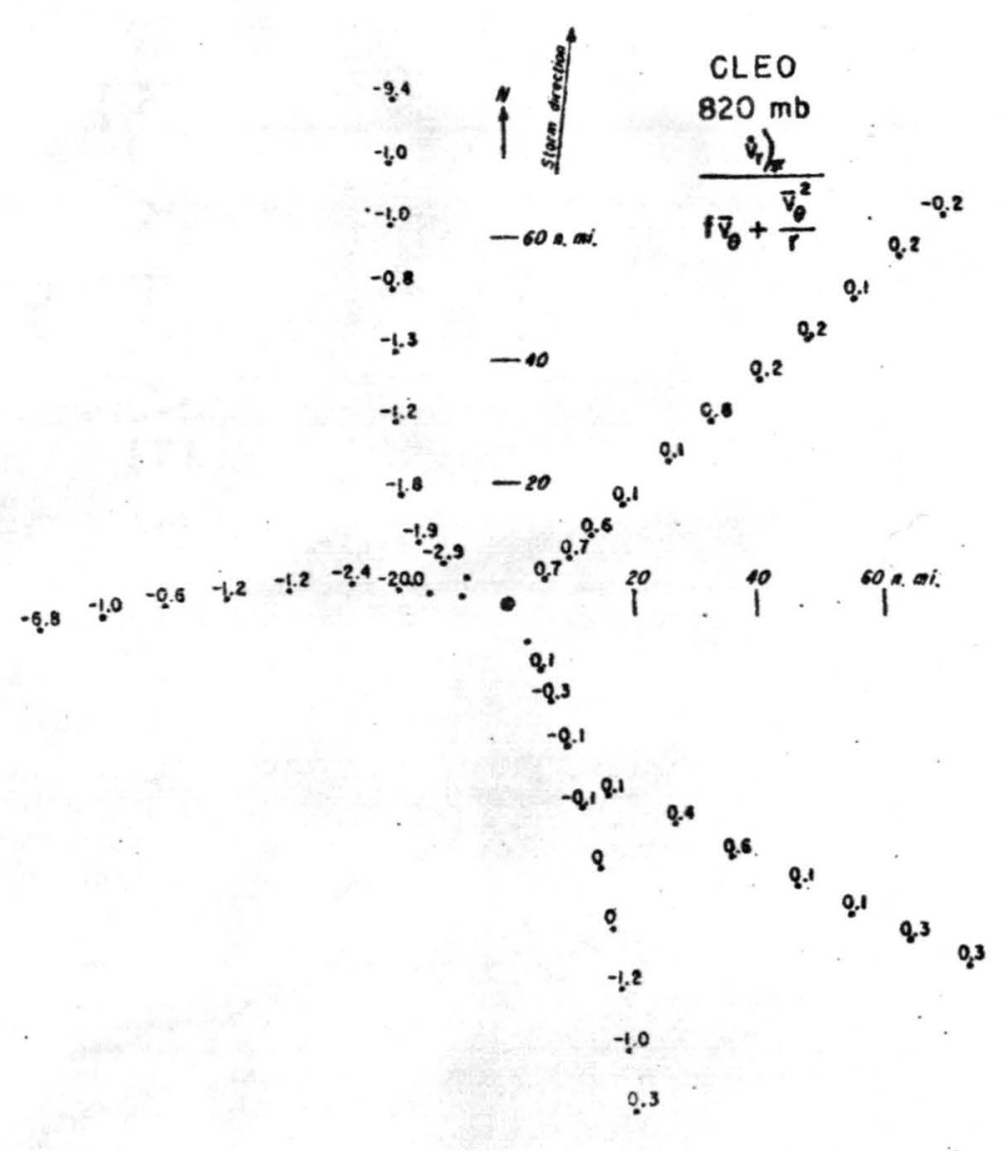

Fig. 31 . 


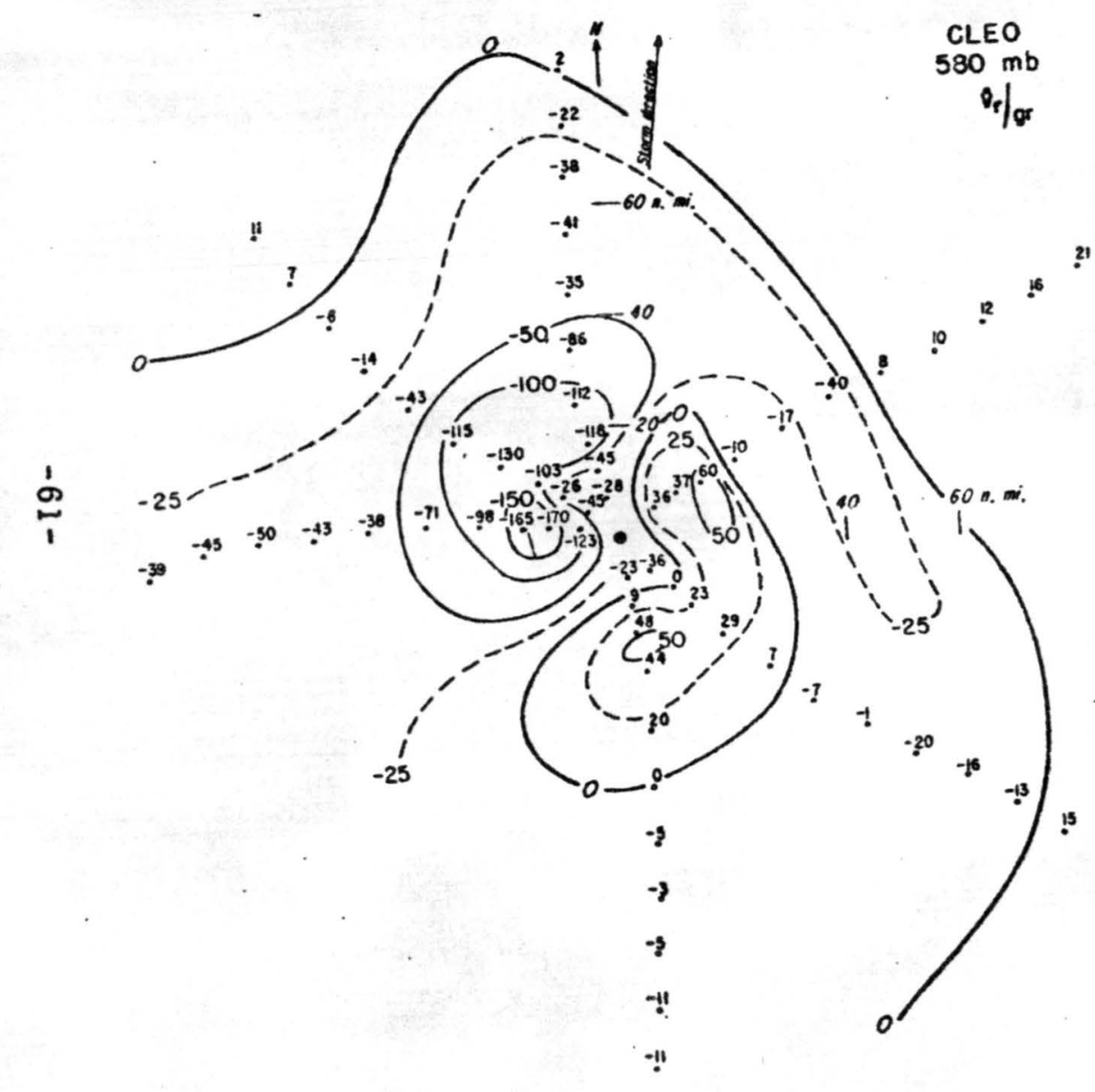

Fig. 32. $\left.\dot{\mathrm{v}}_{\mathbf{r}}\right)_{\mathrm{gr}}$ in knots/hour

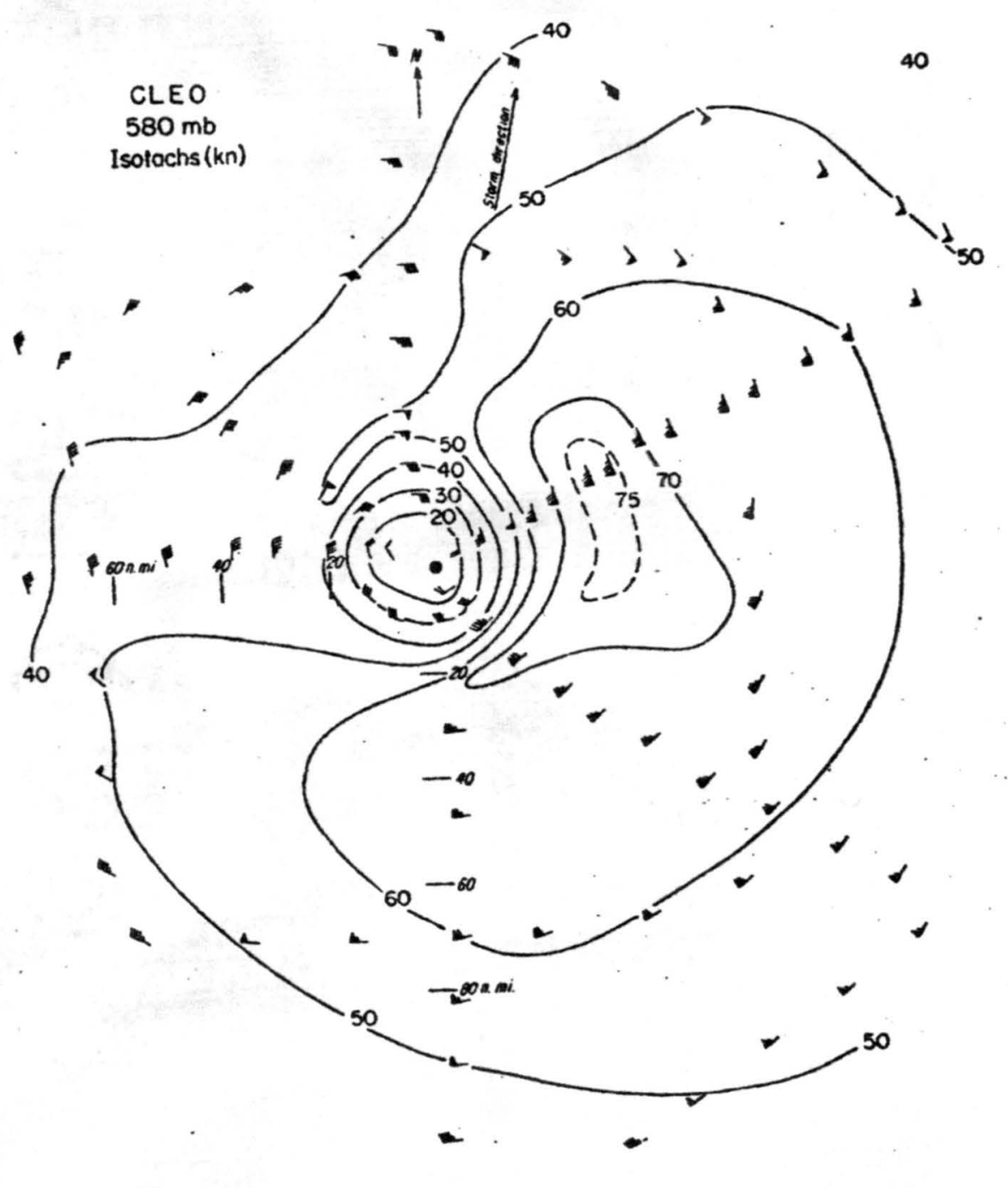

Fig. 33. 


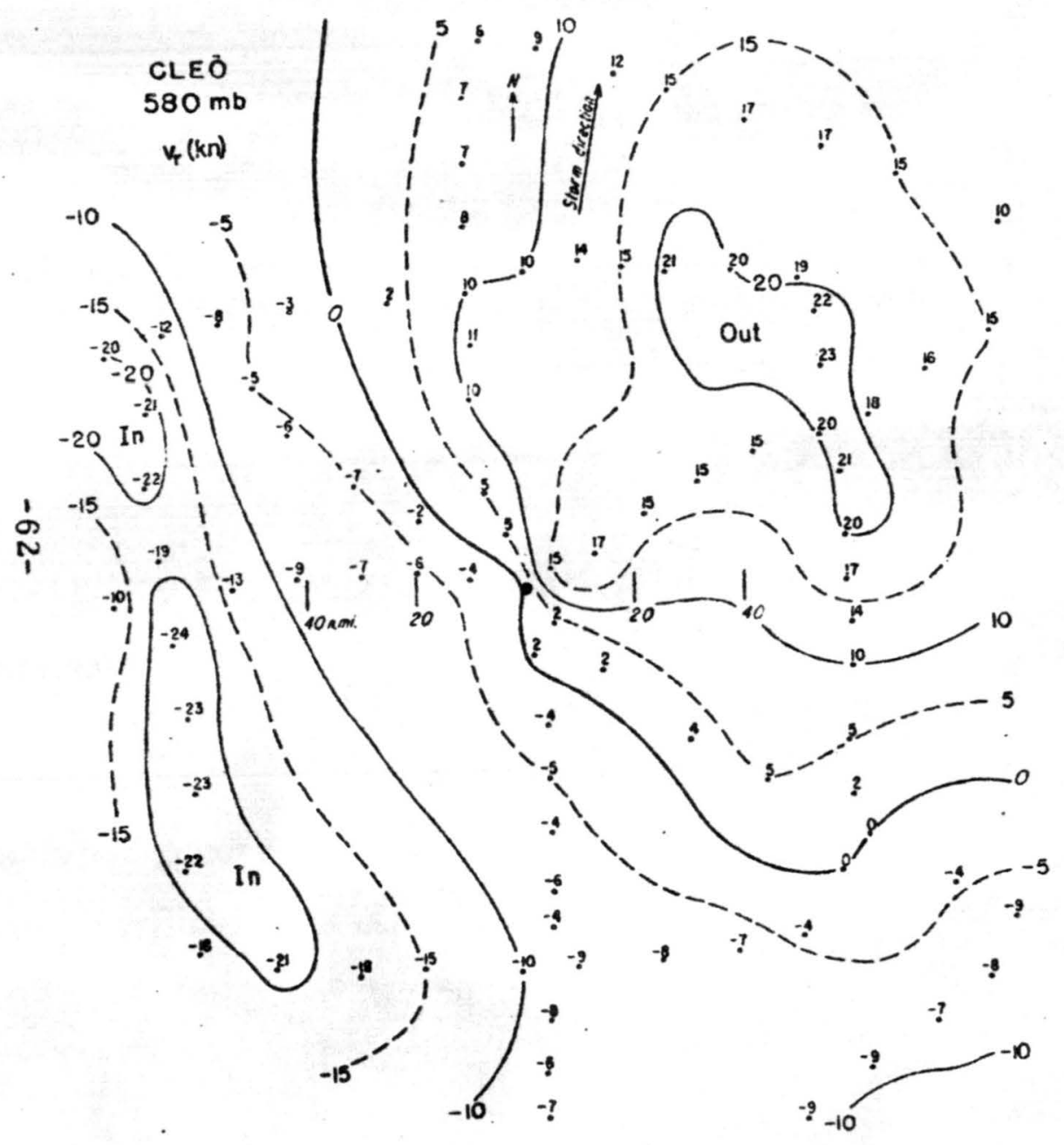

Fig. 34. Radial wind component

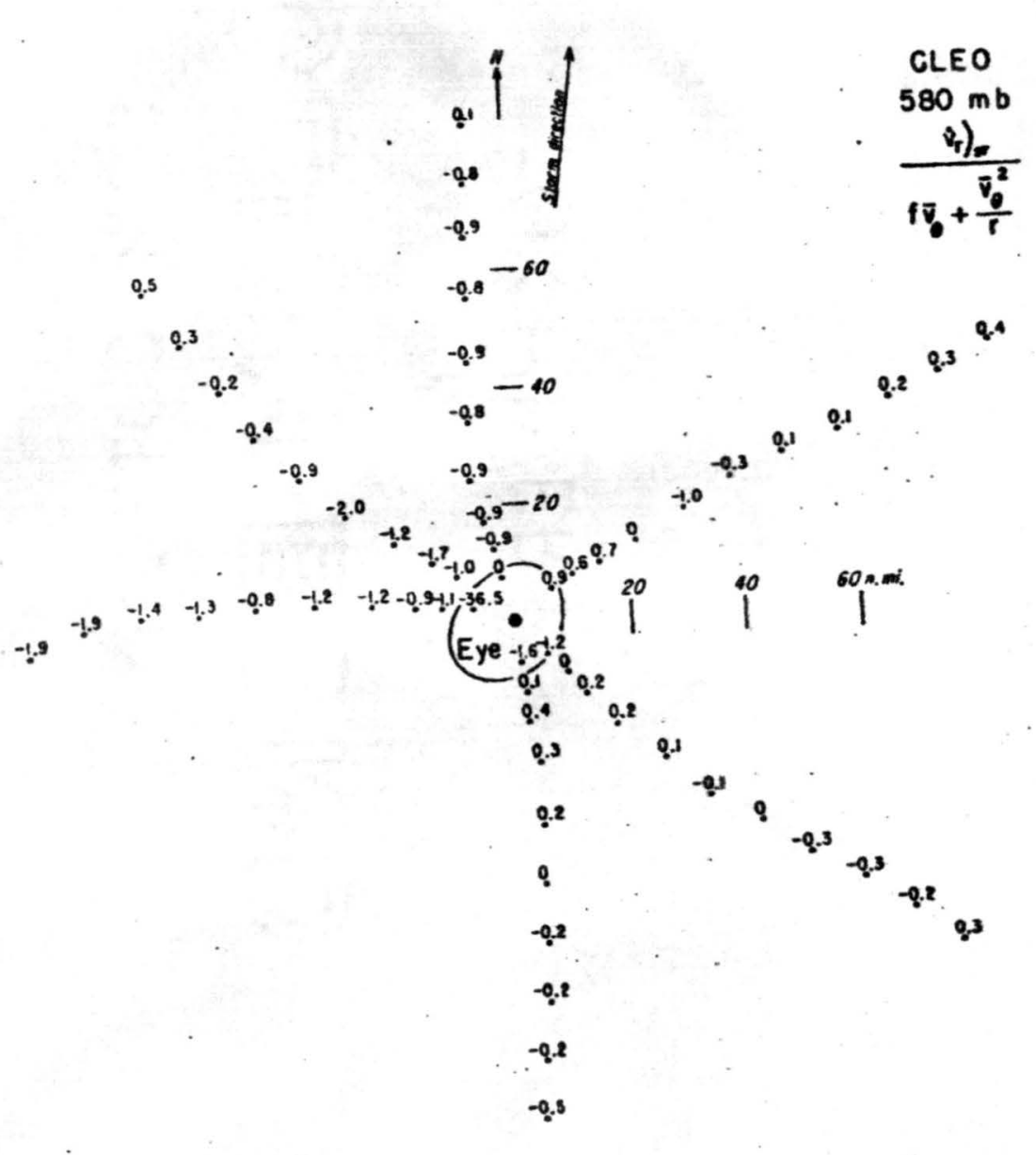

Fig. 35. 


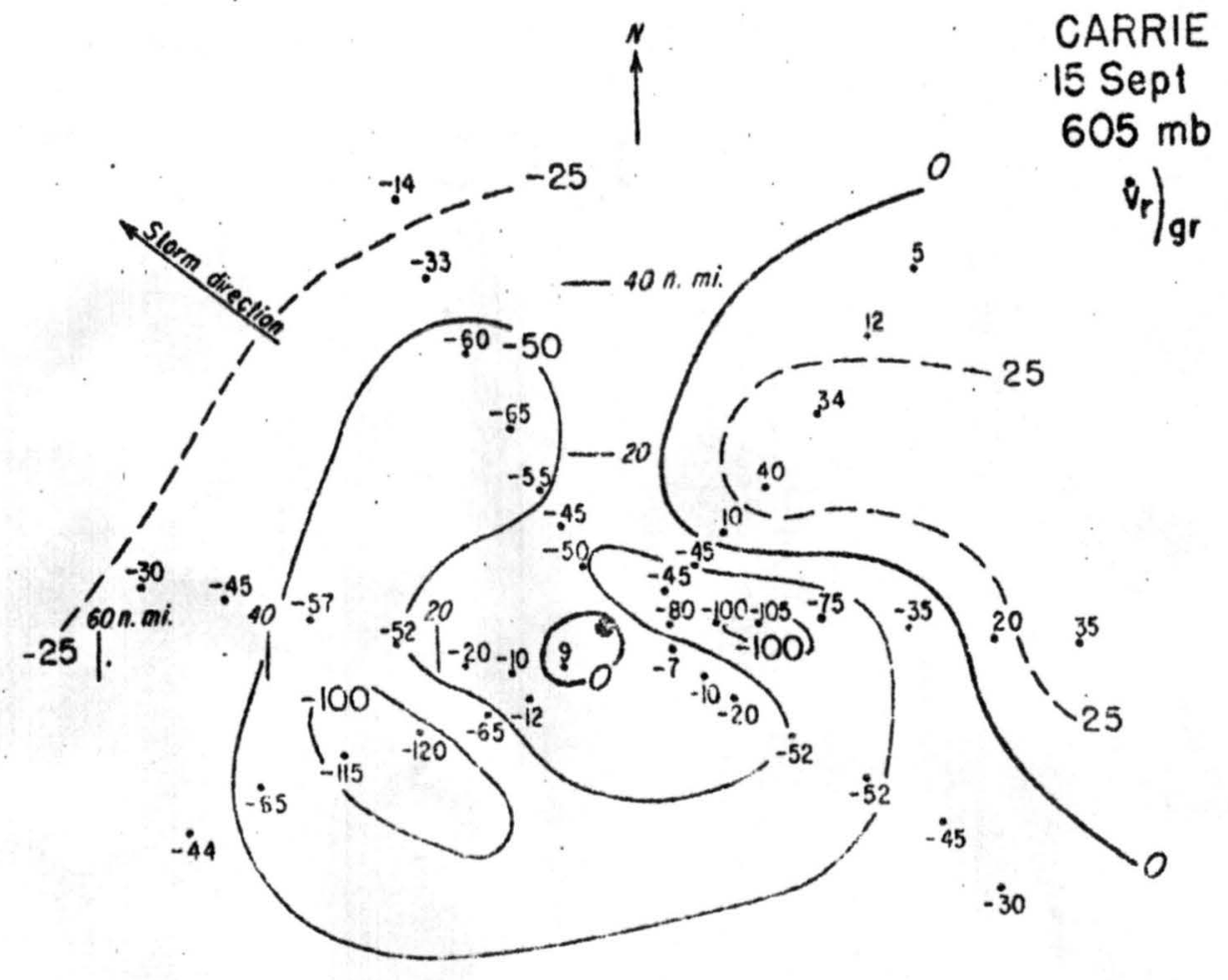

Fig. 36. $\dot{\mathrm{v}}_{\mathrm{r}} \mathrm{g}_{\mathrm{gr}}$ in knotsinour

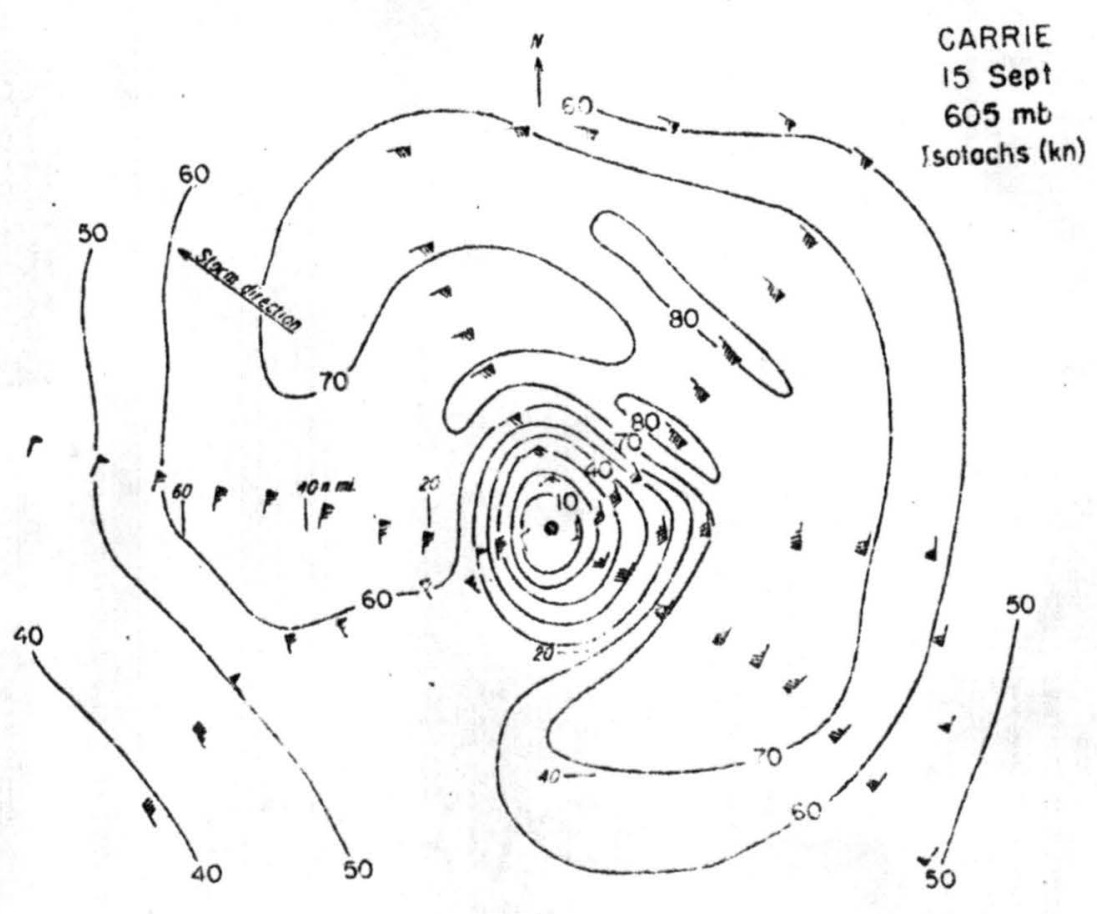

Fig. 37 . 


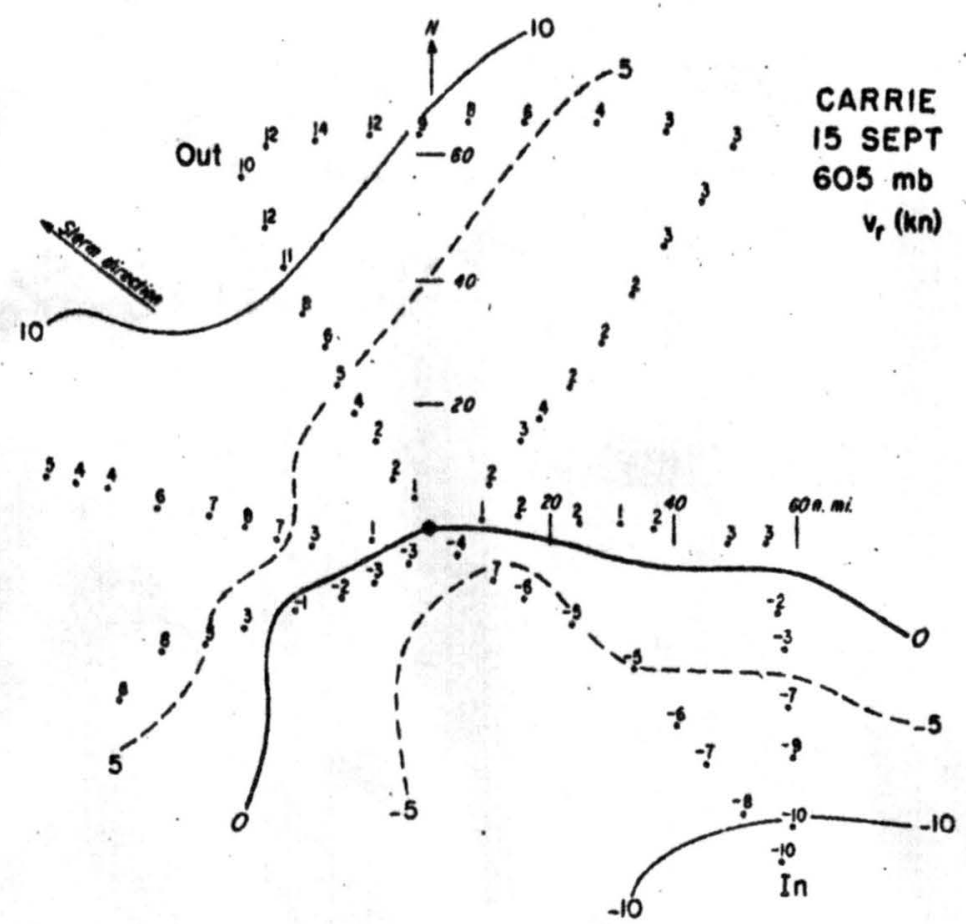

Fig. 38. Radial wind component

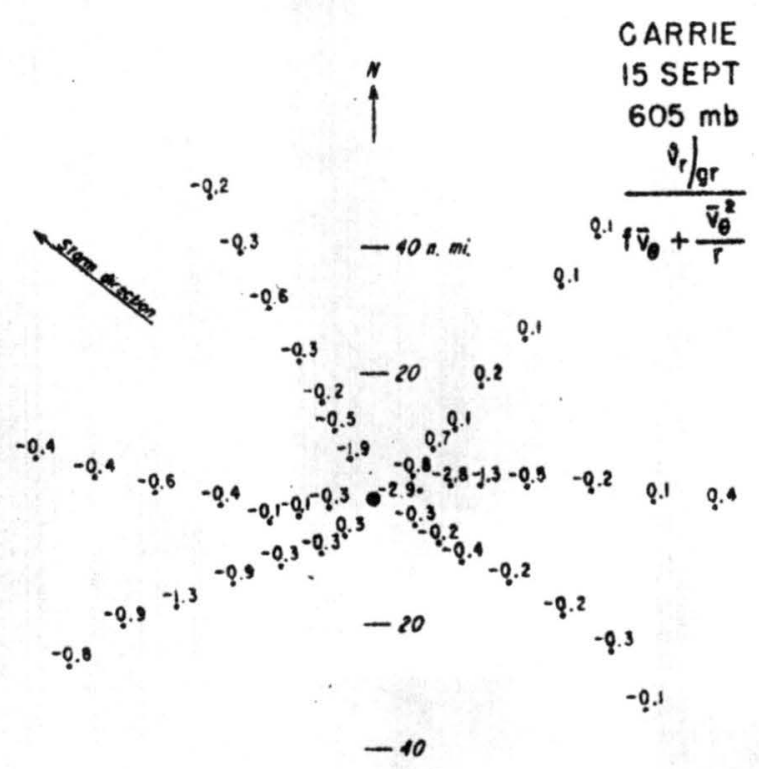

Fig. 39. 


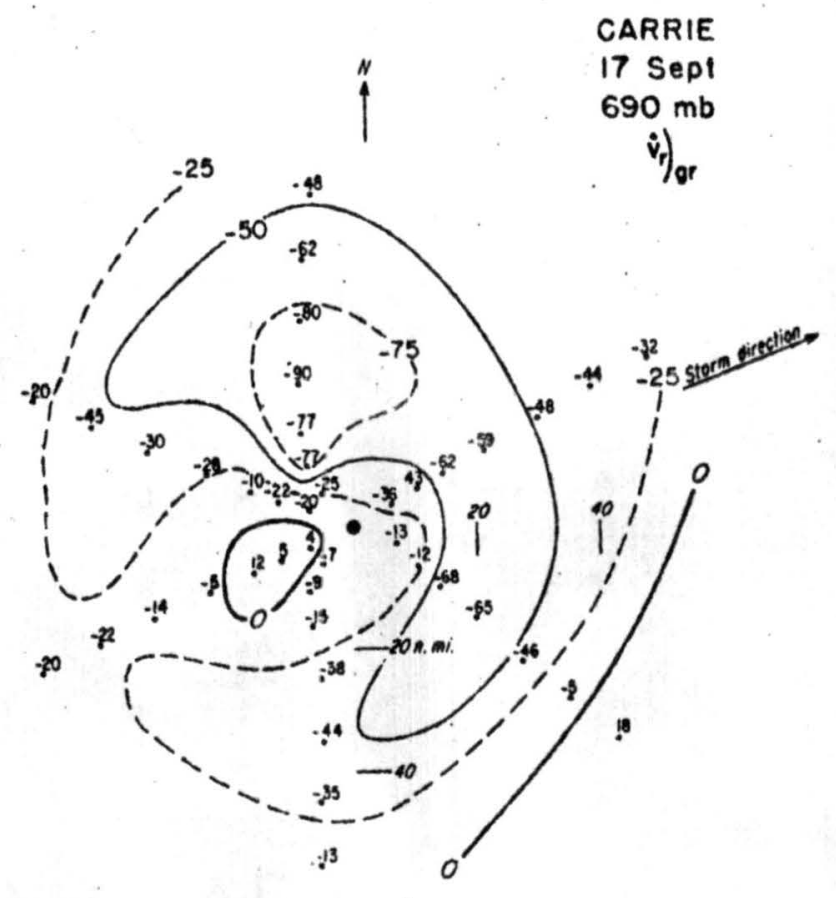

Fig. 40. $\dot{\mathrm{v}}_{\mathrm{r}} \mathrm{gr}_{\mathrm{g}}$ in knots/hour

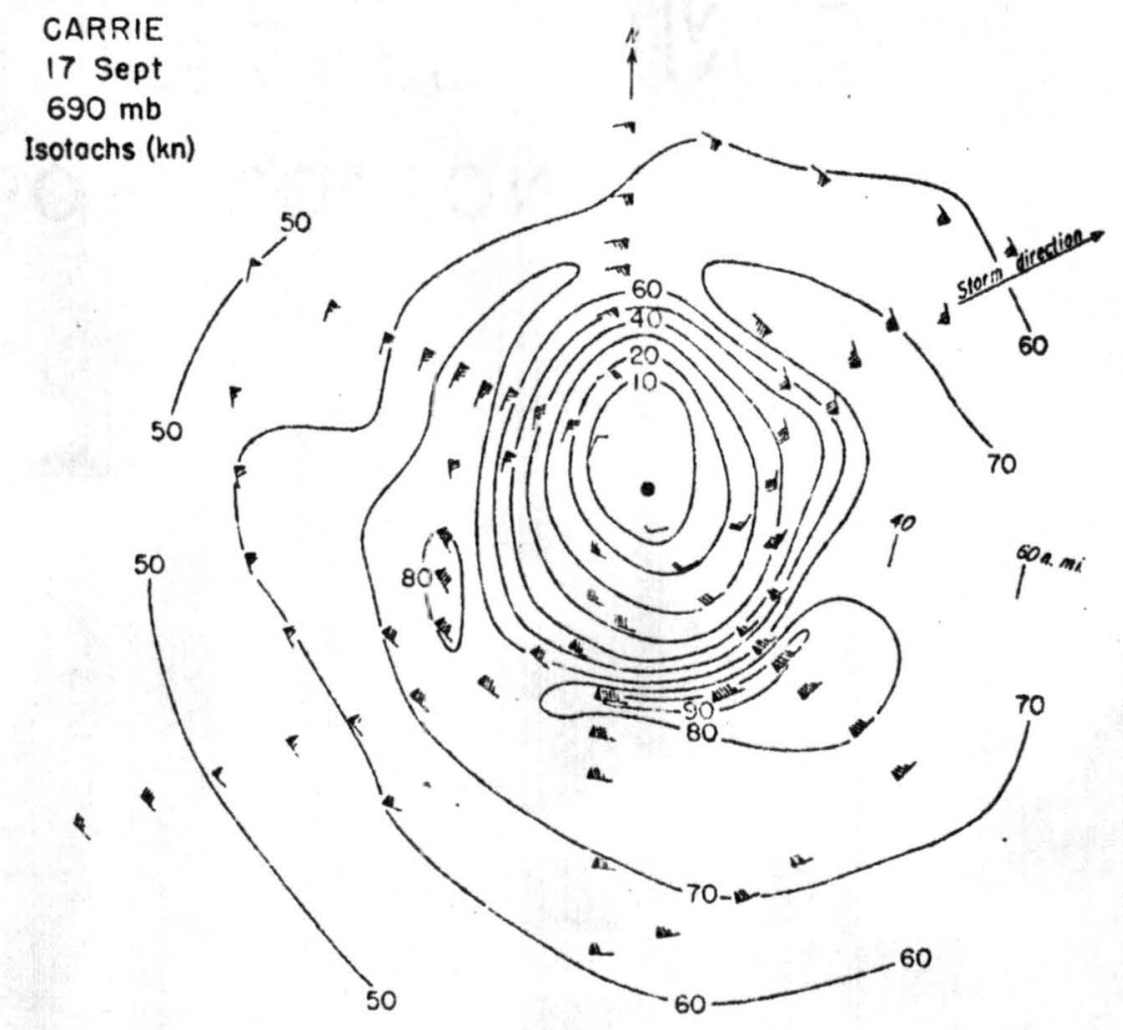

Fig. 41 . 


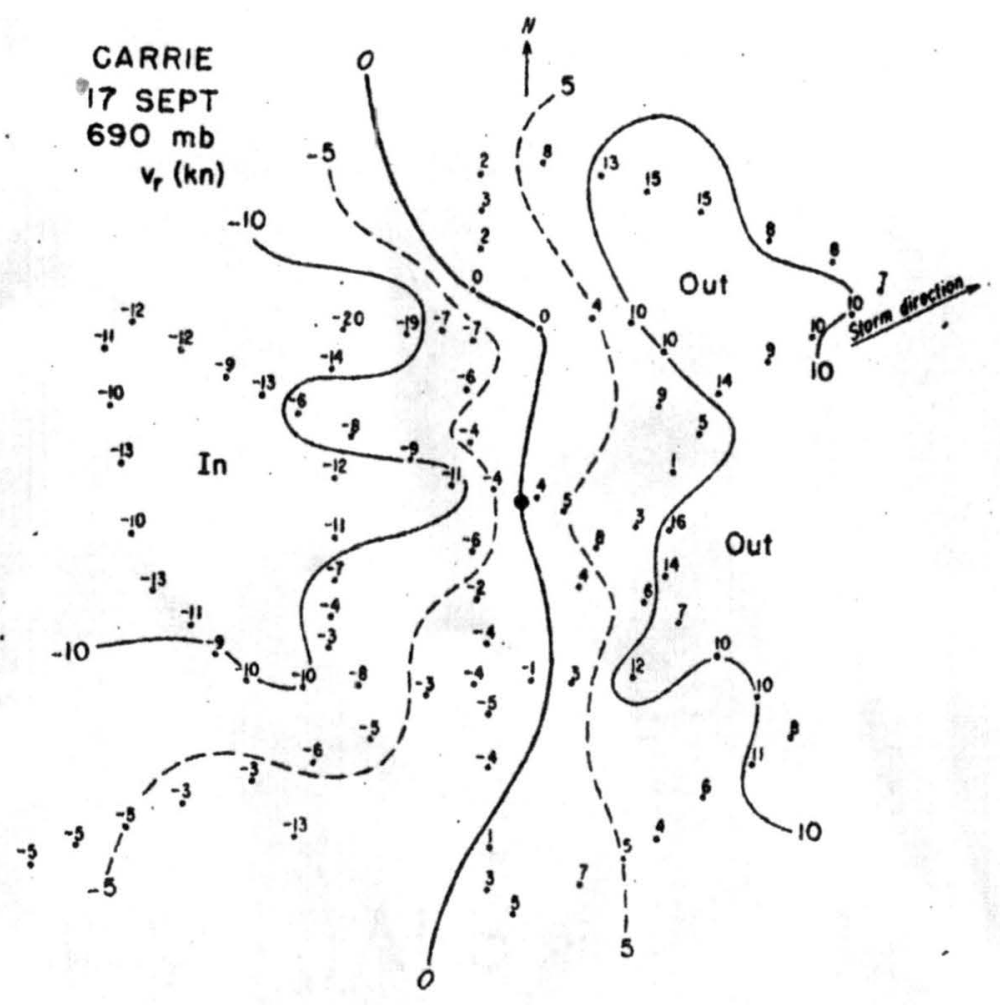

Fig. 42. Radial wind component

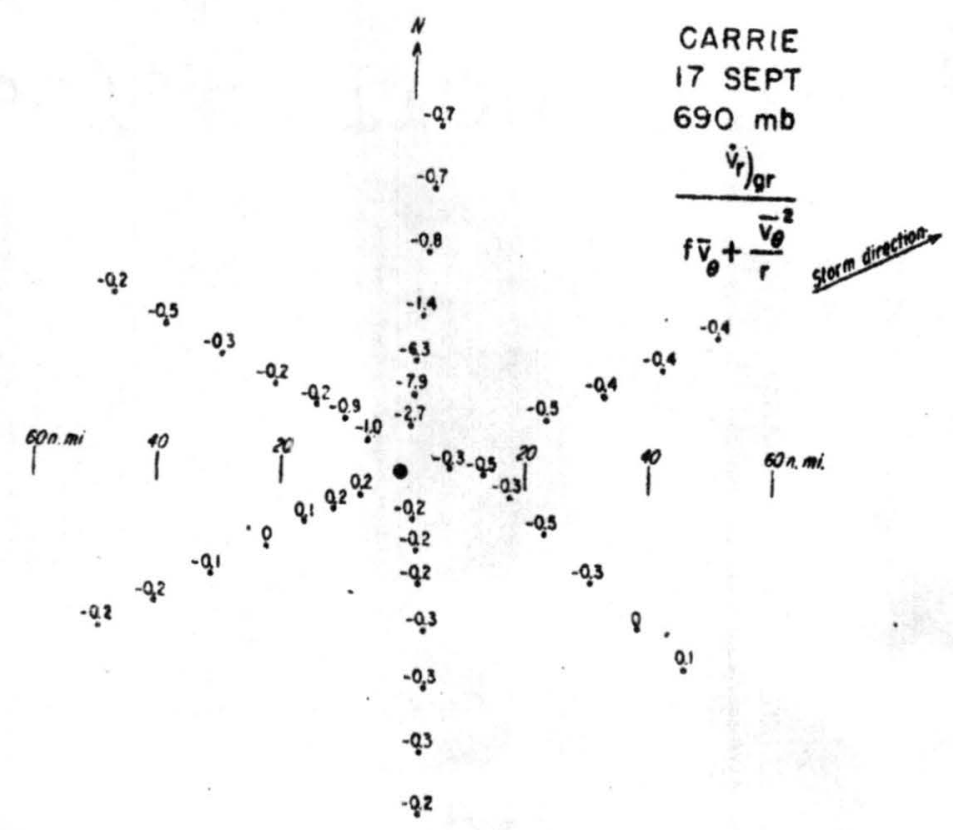

Fig. 43. 


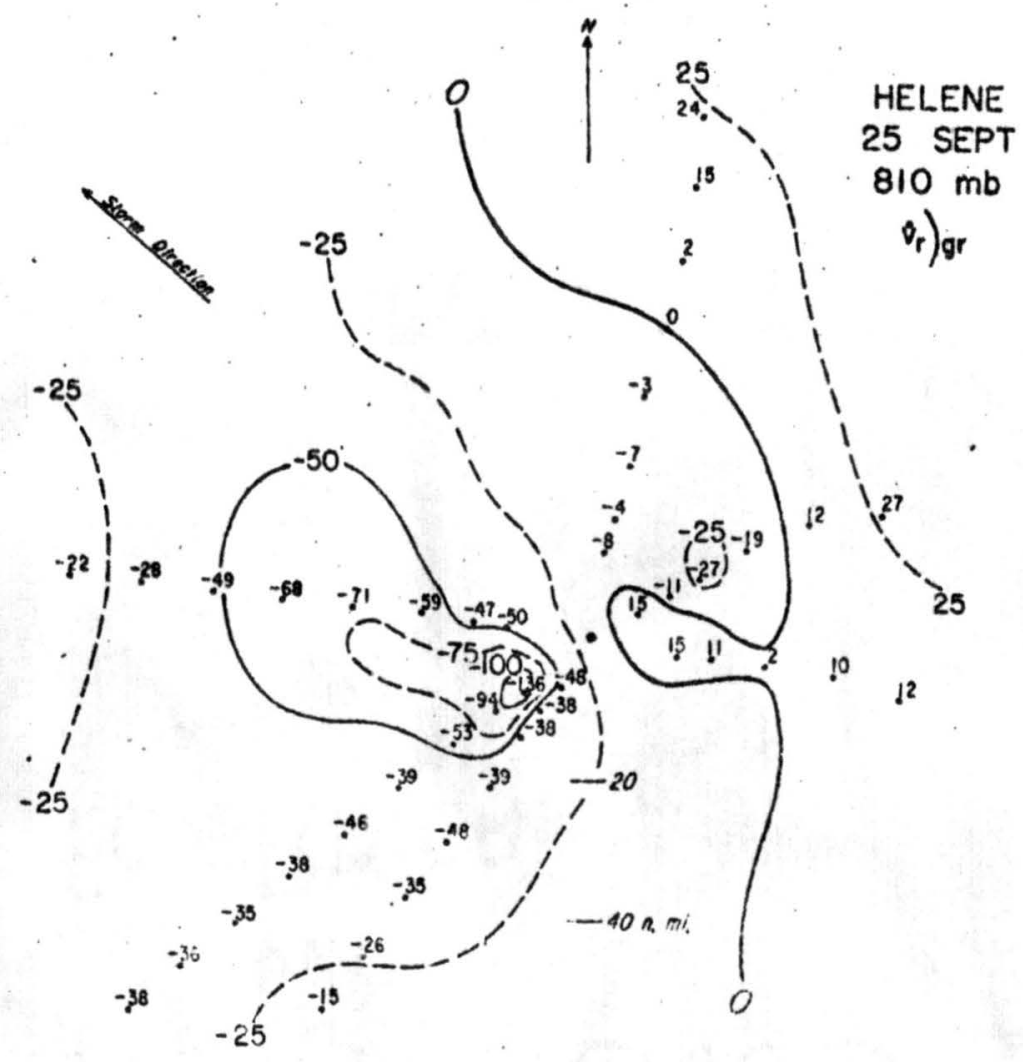

Fig. 44. $\dot{v}_{\mathrm{r}}{ }_{\mathrm{gr}}$ in knots/hour

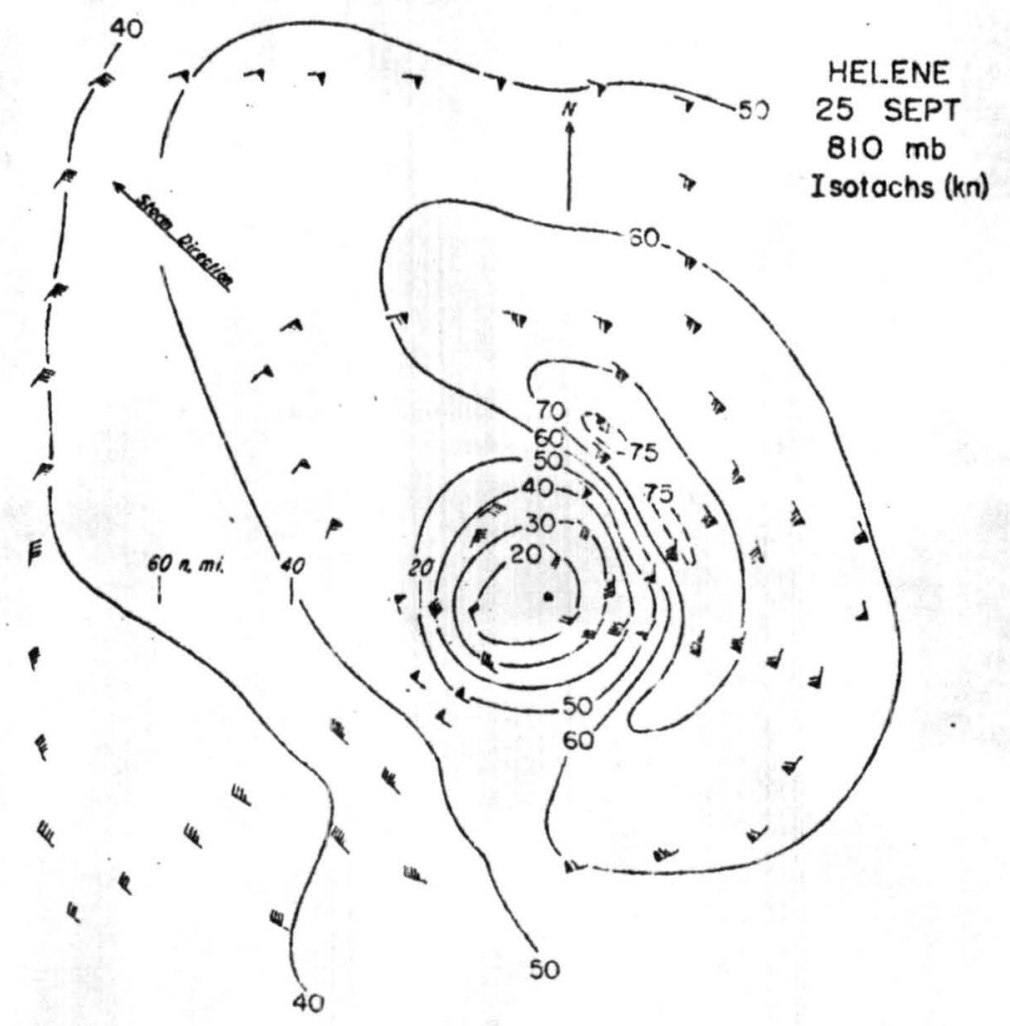

Fig. 45. 


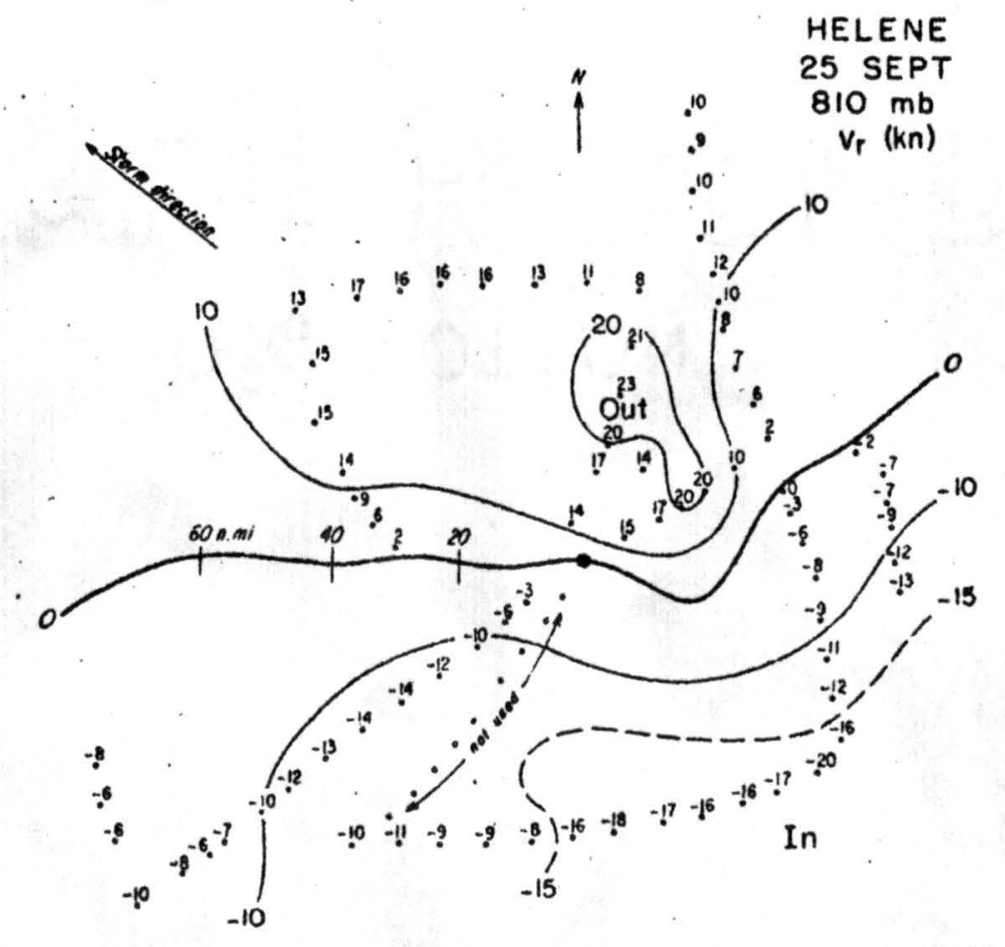

Fig. 46. Radial wind component

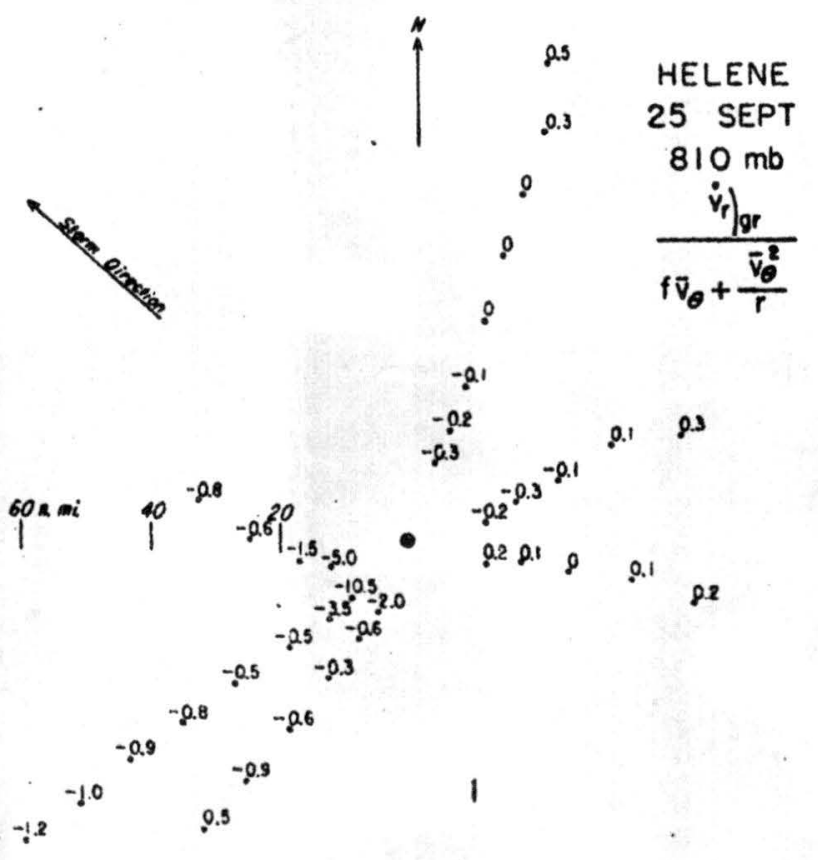

Fig. 47. 


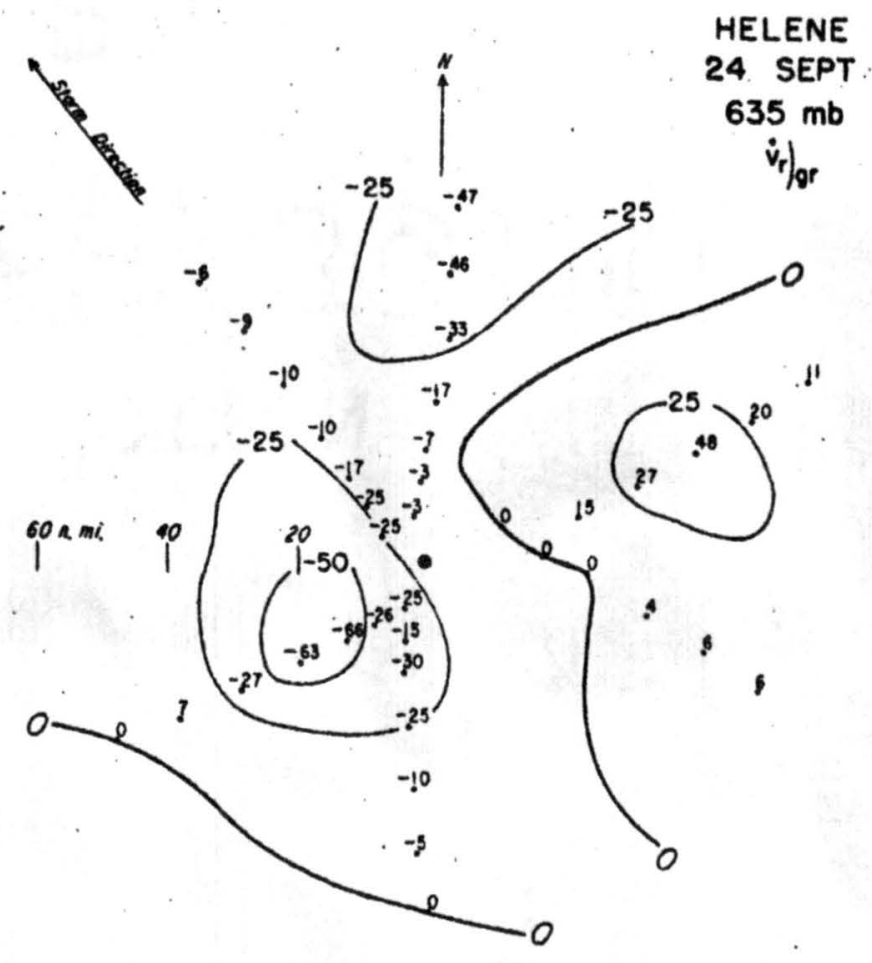

Fig. 48. $\left.\dot{\mathrm{v}}_{\mathrm{r}}\right)_{\mathrm{gr}}$ in knots/hour

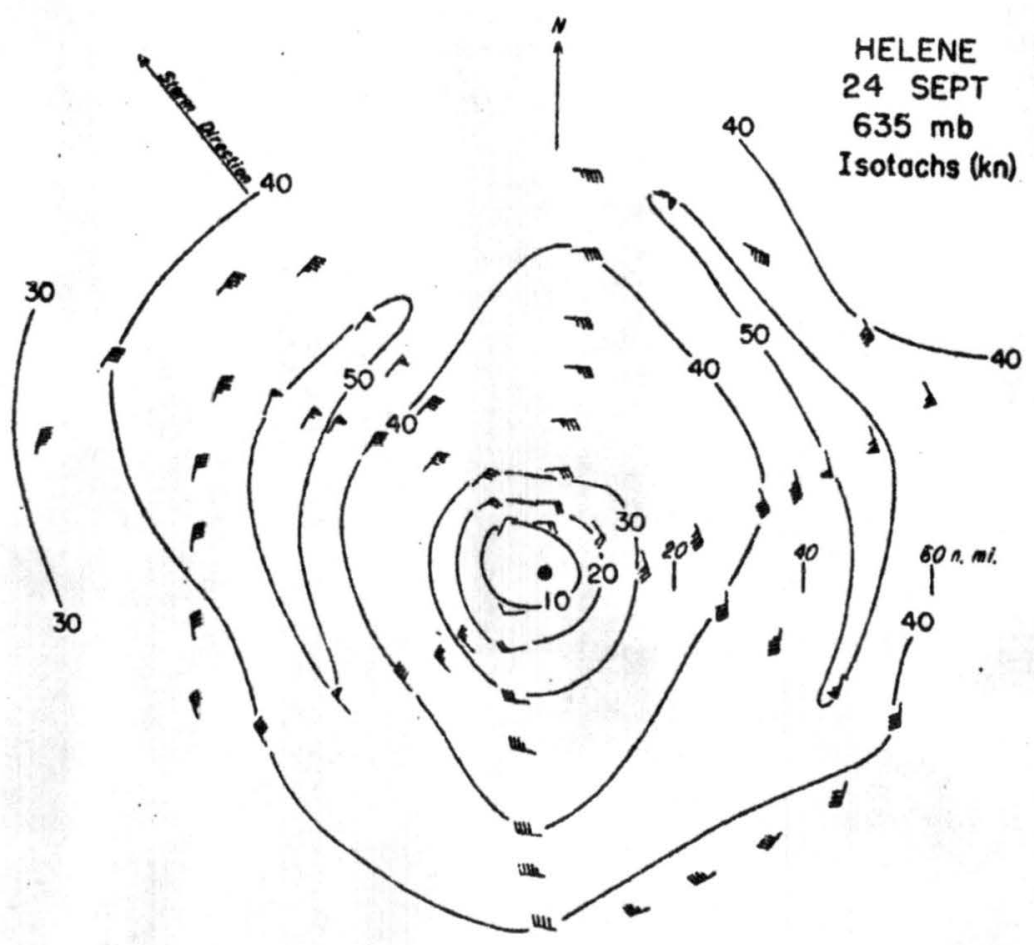

Fig. 49 .

$-69-$ 


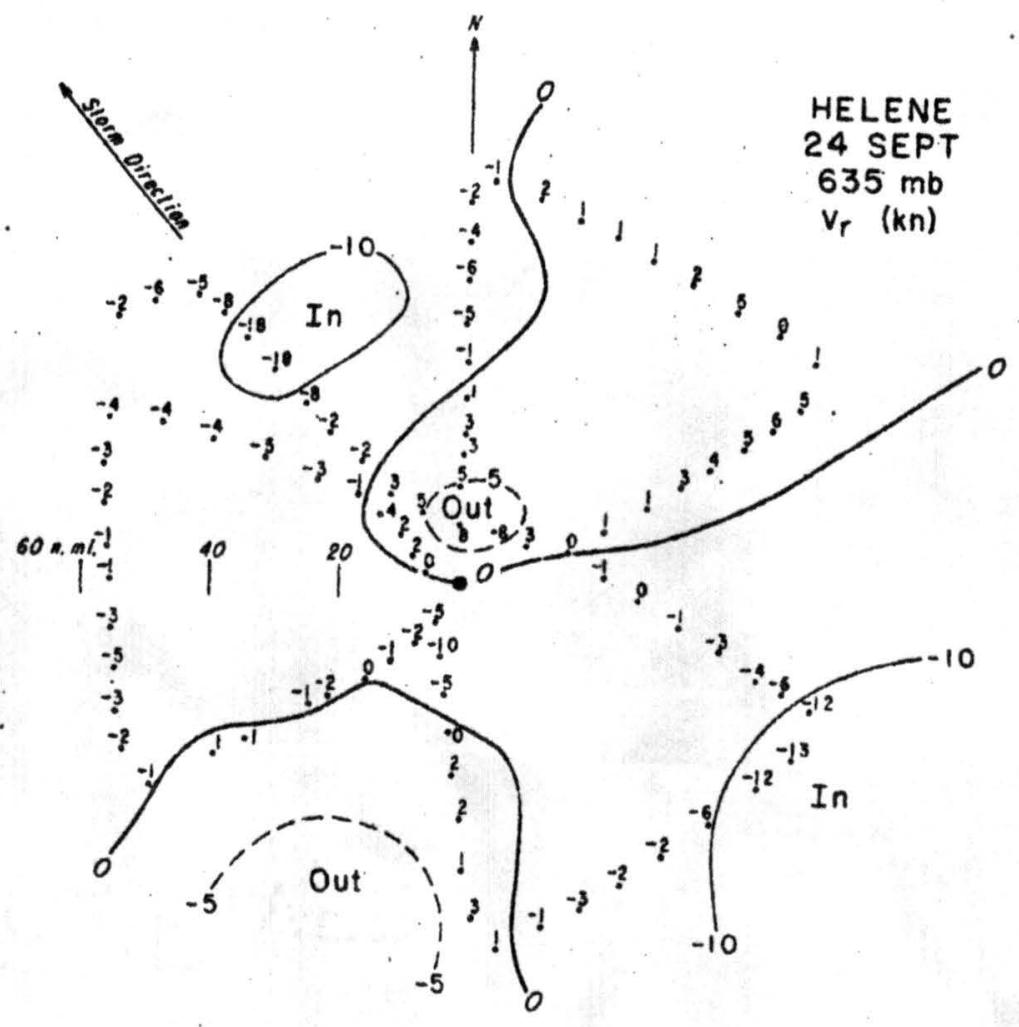

Fig. 50. Radial wind component

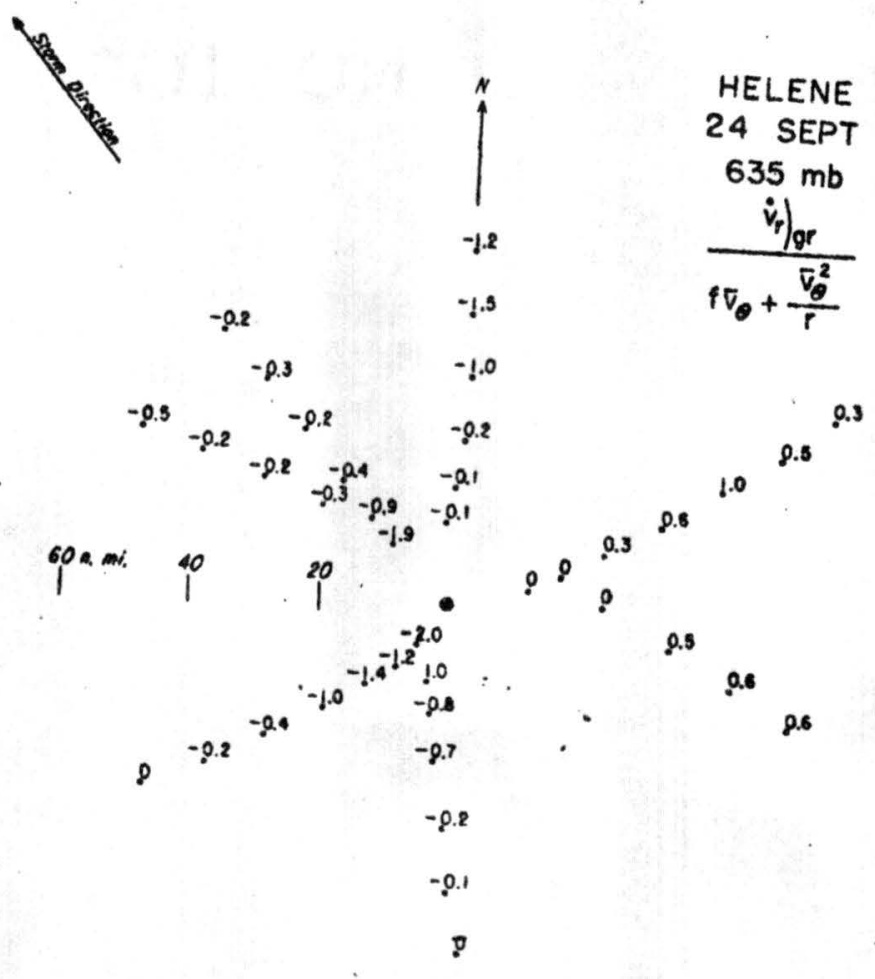

Fig. 51 . 


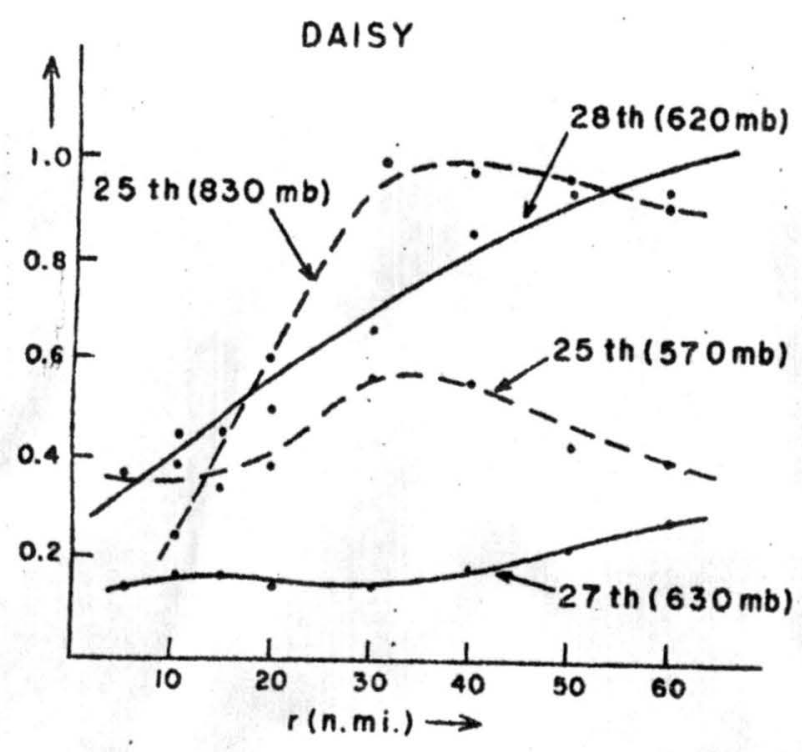

Fig. 52.

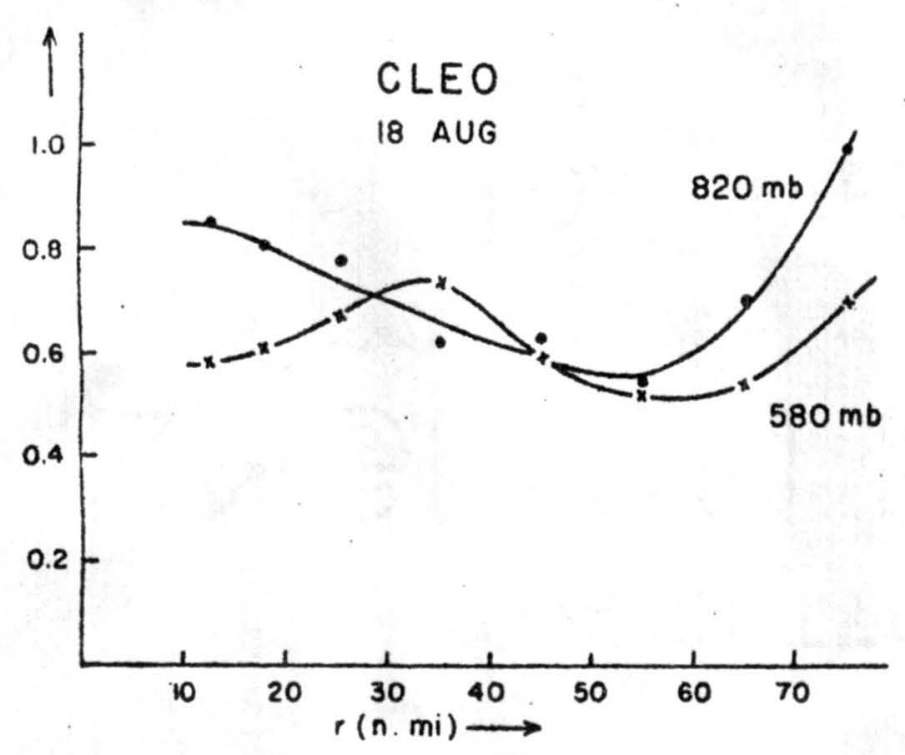

Fig. 54.

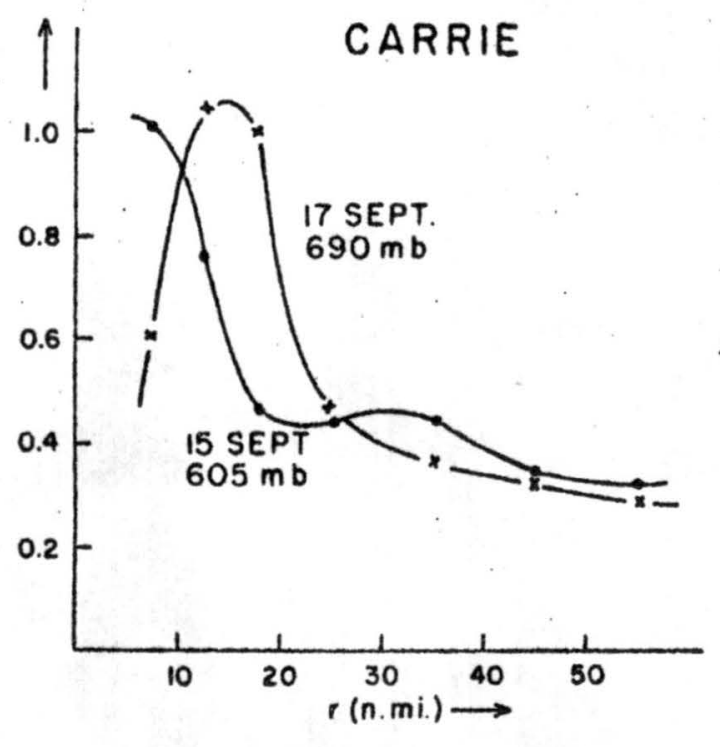

Fig. 53.

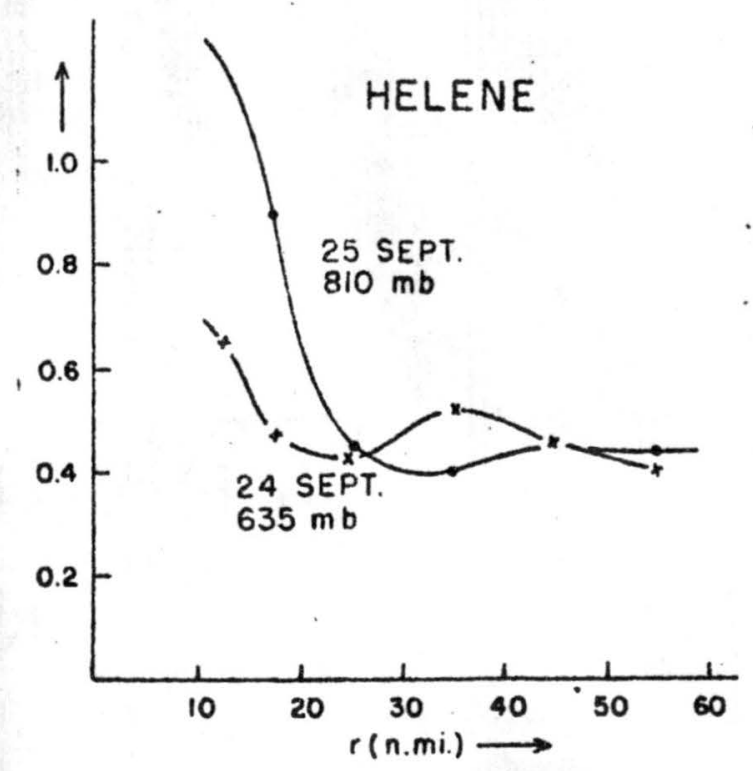

Fig. 55.

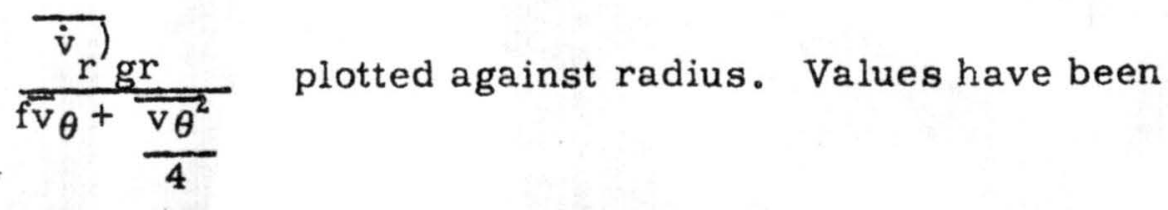

averaged around the storm without respect to the sign

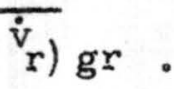




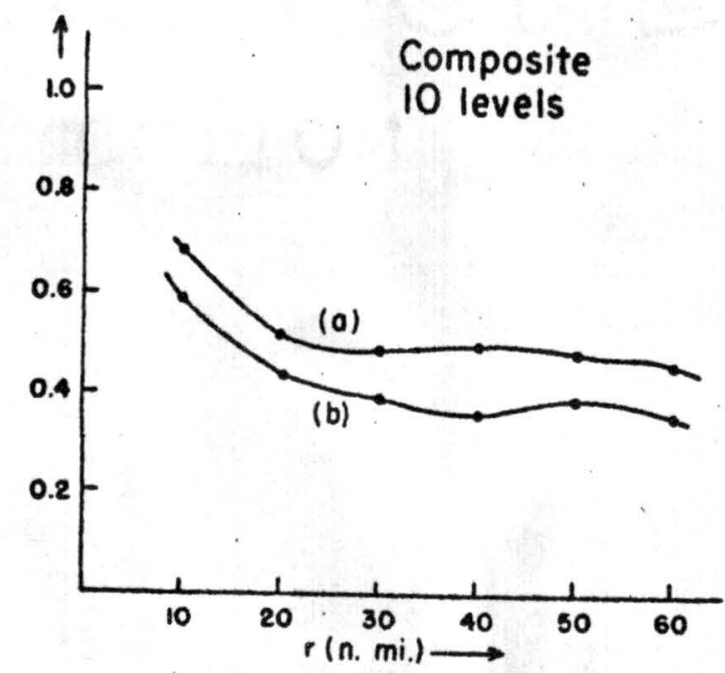

Fig. 56.
(a) $\frac{\overline{\dot{v}_{r}{ }^{\prime} g r}}{f \bar{v}_{\theta}+\frac{v_{\theta}^{2}}{r}}$
(b) $\frac{\dot{\mathrm{v}}_{r}{ }_{\mathrm{gr}}}{\mathrm{g} \frac{\partial \mathrm{D}}{\partial r}}$

Values are plotted against radius after averaging around the storm without respect to the sign of $\dot{\mathrm{v}}_{\mathrm{r}}{ }^{\prime} \mathrm{gr}$ 


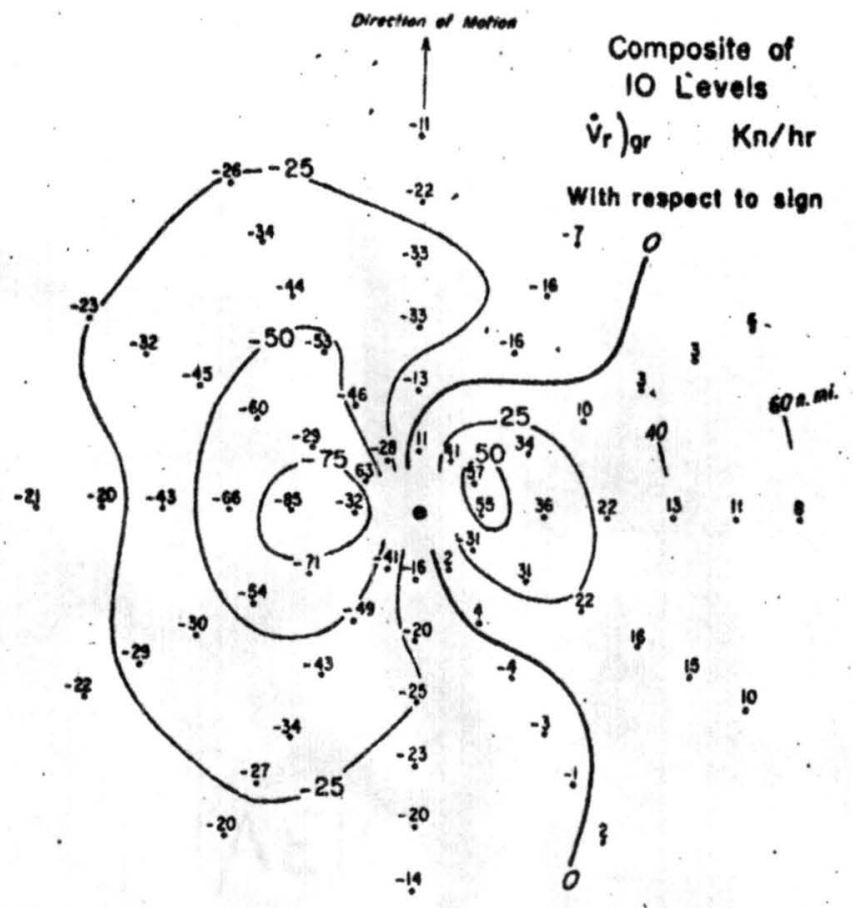

Fig. 57 .

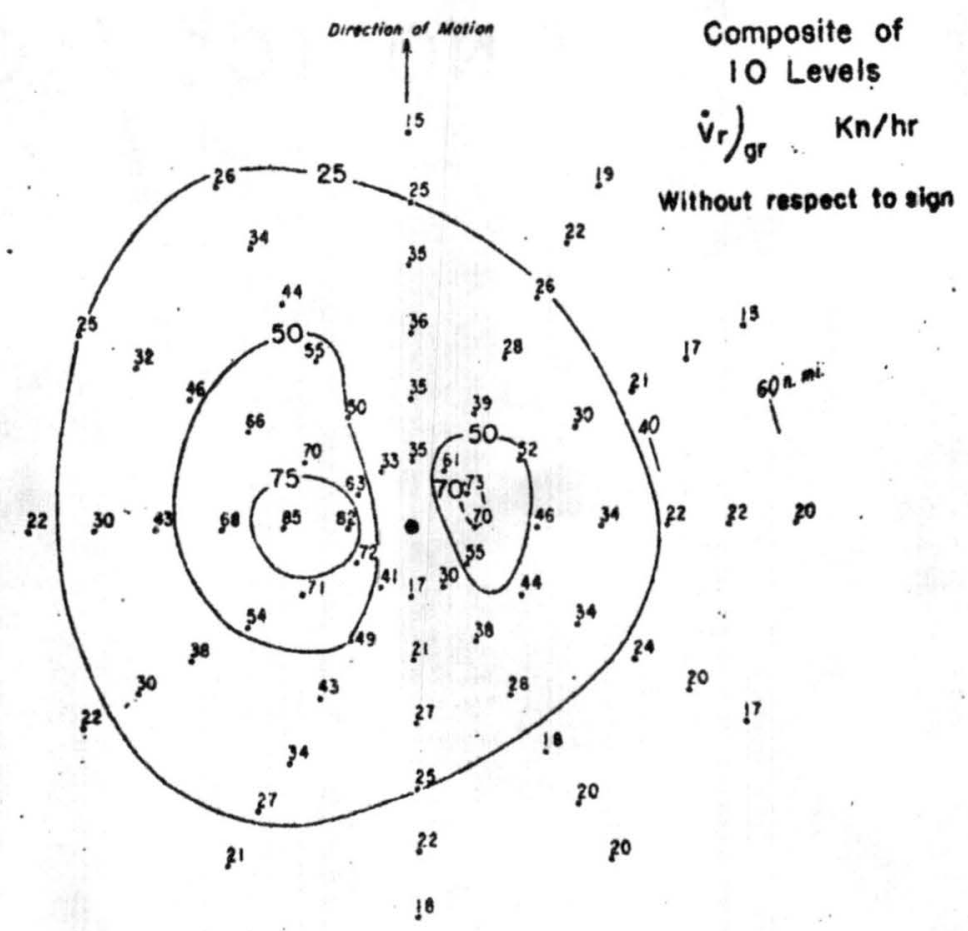

Fig. 58 . 


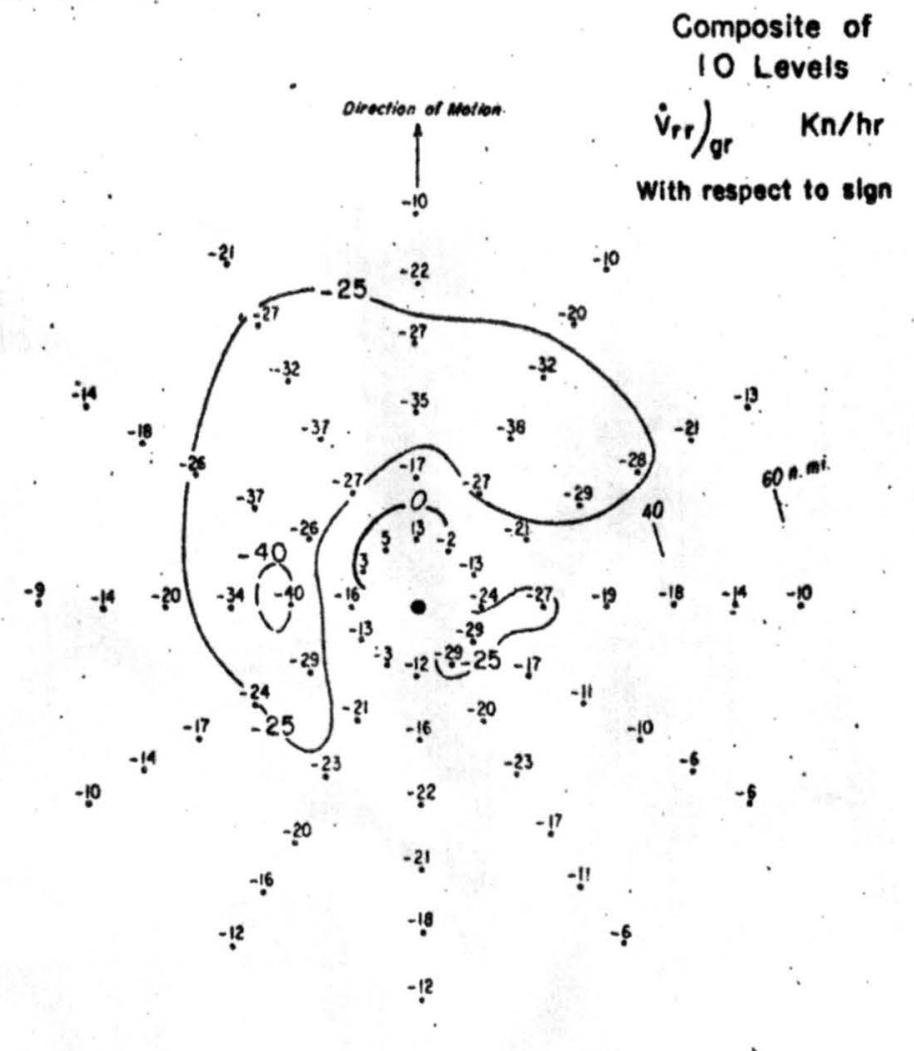

Fig. 59.

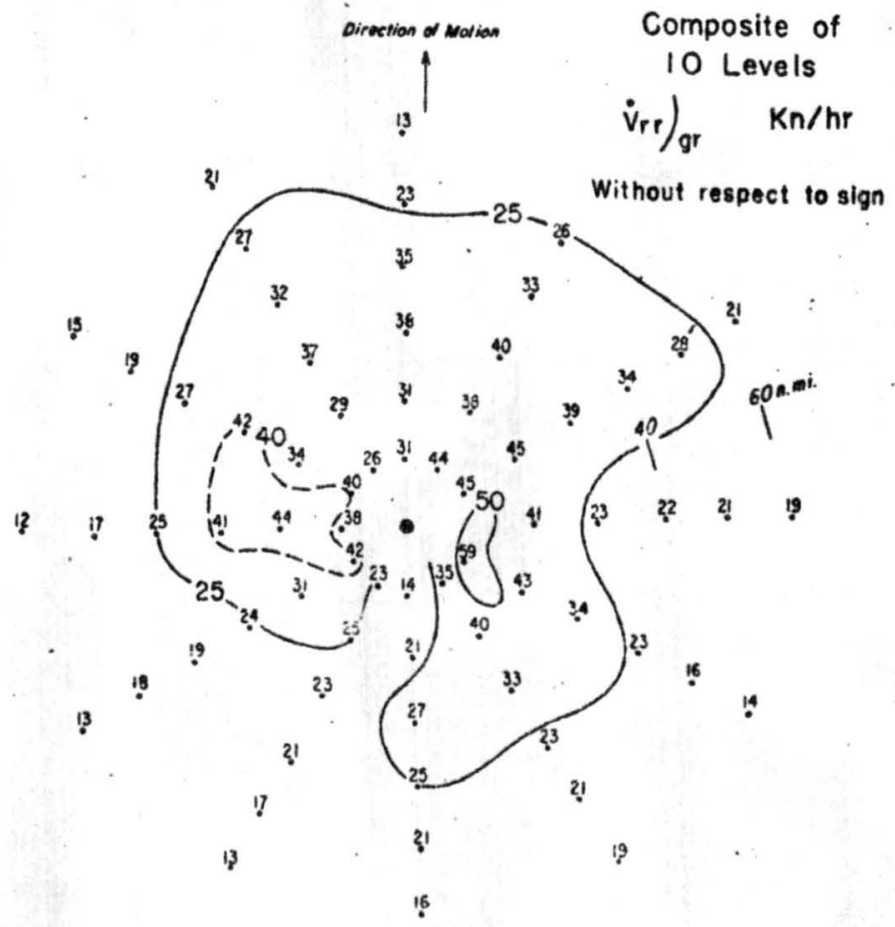

Fig. 60 . 


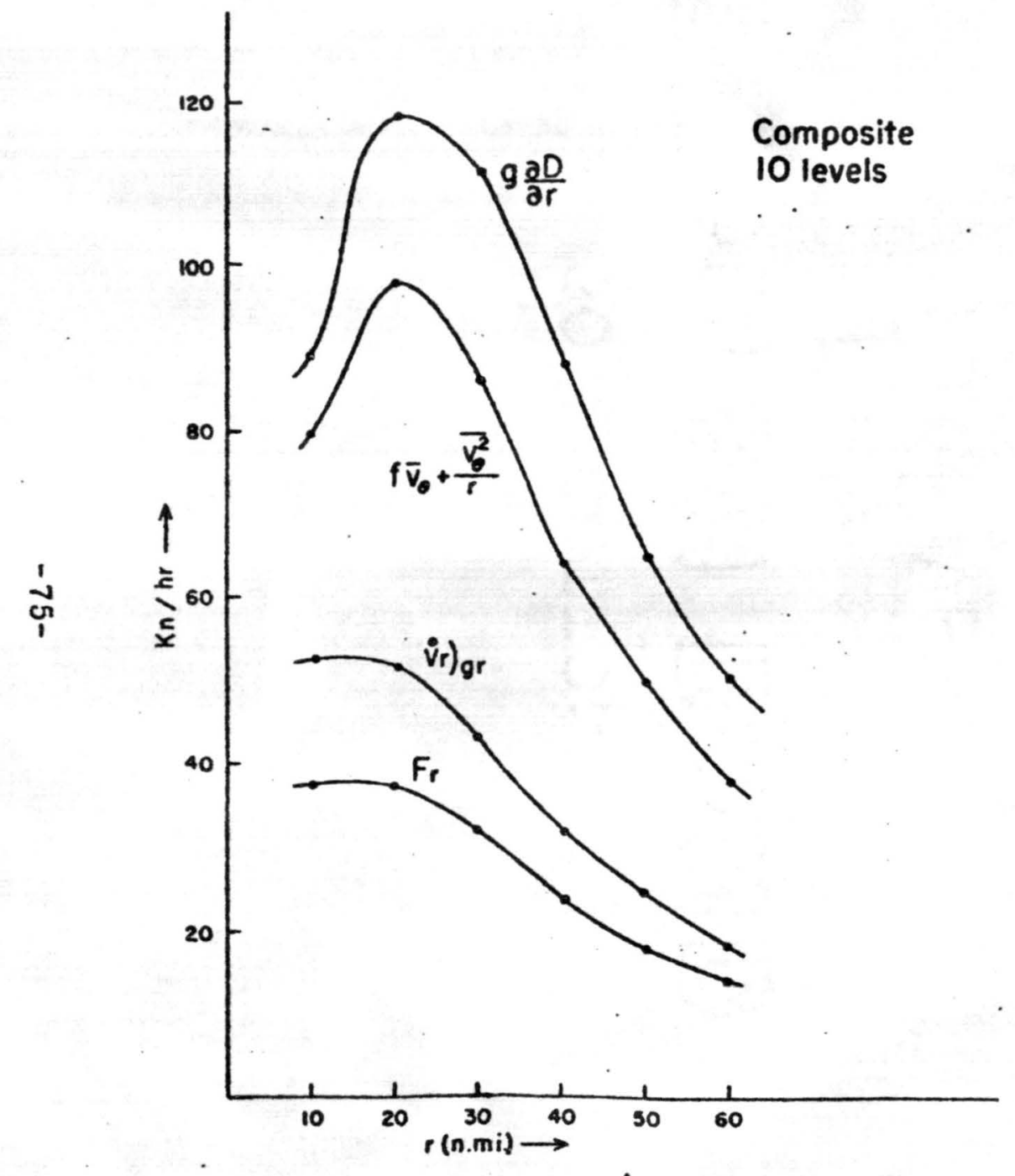

Fig. 61 .

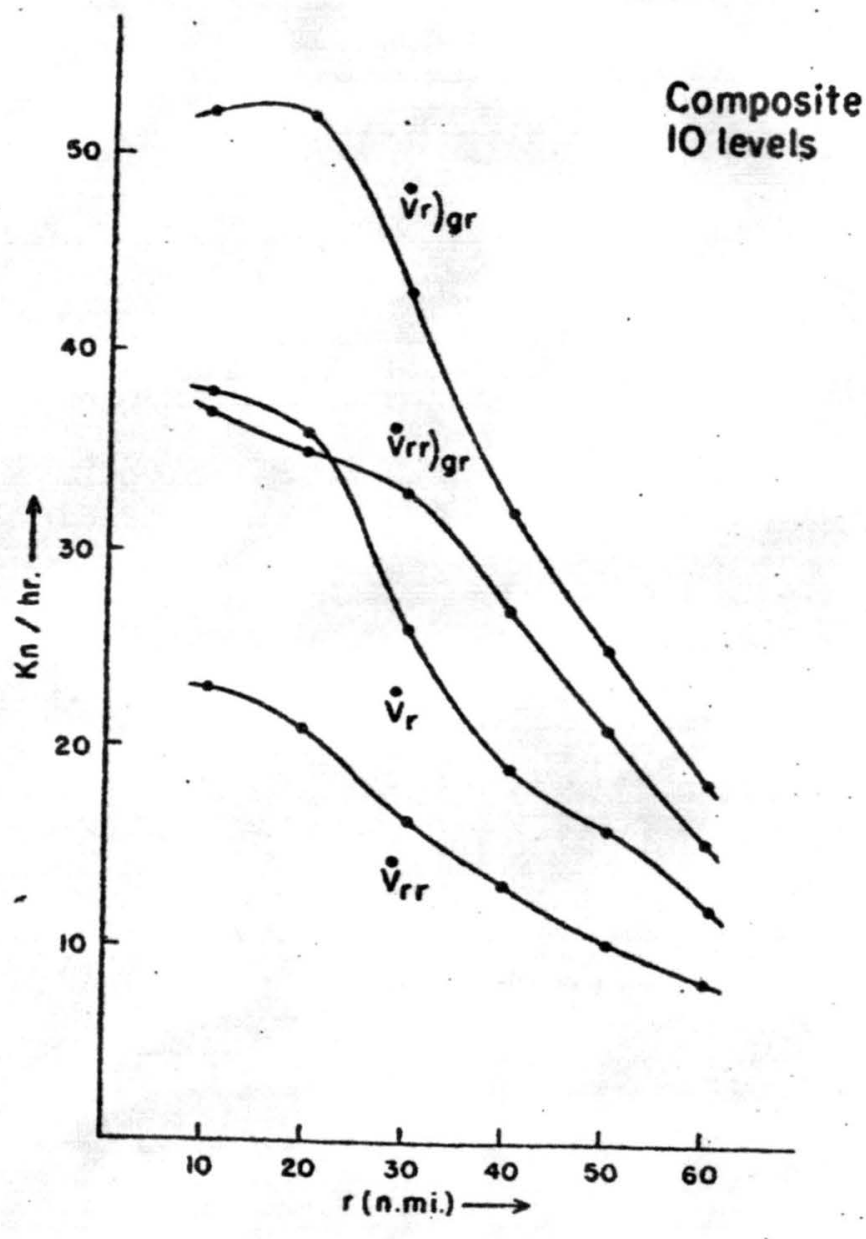

Fig. 62 .

Radial profiles of various accelerations at selected radii. Values have been averaged around storn with ut respect to sign. 


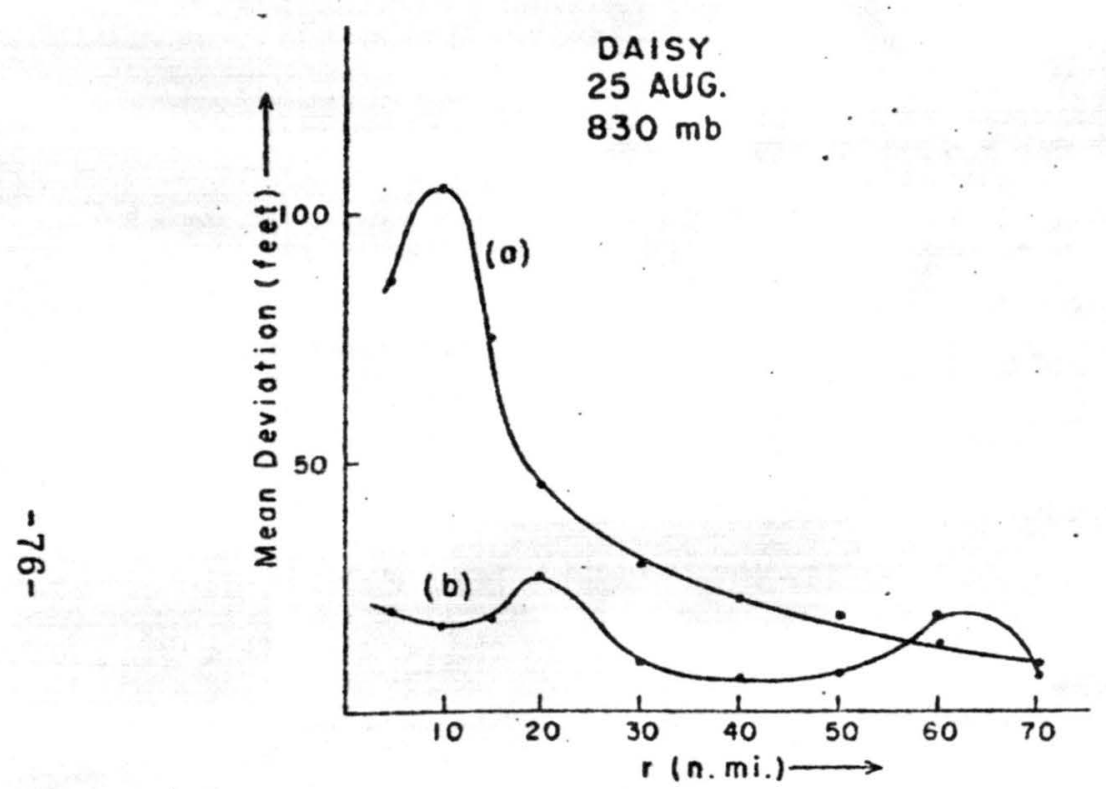

Fig. 63. $830 \mathrm{mb}, 25$ Aug.

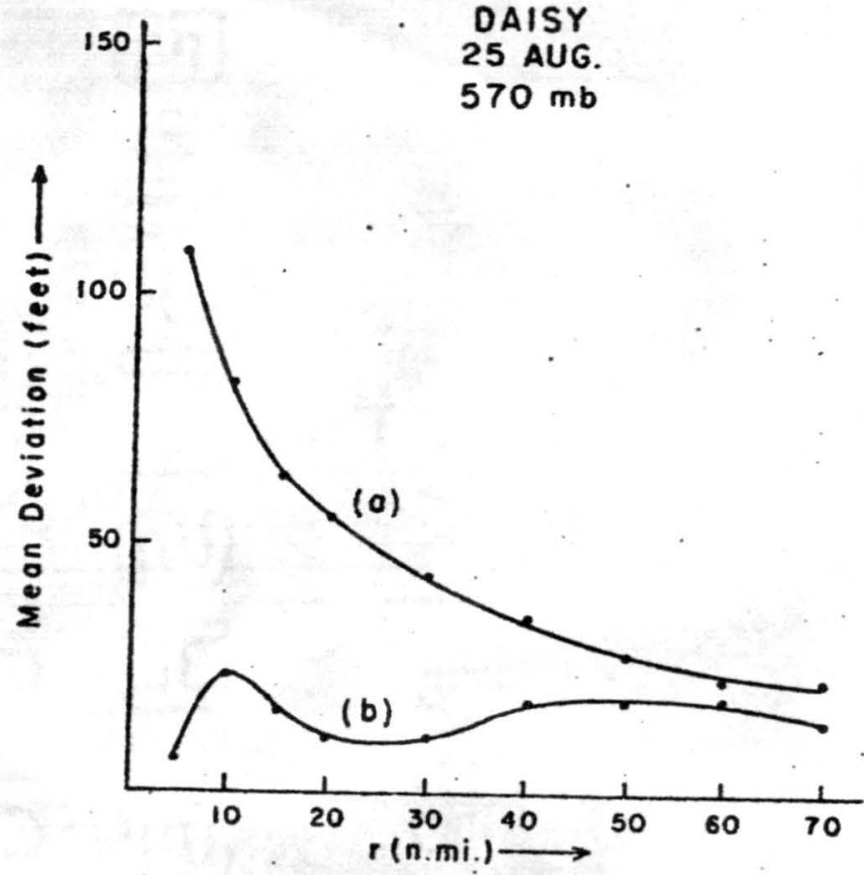

Fig. 64. $570 \mathrm{mb}, 25$ Aug.

Curve (a) -- Mean deviation of the $\mathrm{D}_{\mathrm{gr}}$ profiles of the individual radial flight legs at selected radil from their mean value (feet).

Curve (b) -- Mean deviation of the $D_{\text {act }}$ profiles of the individual radial flight legs at selected radii from their mean value (feet). 


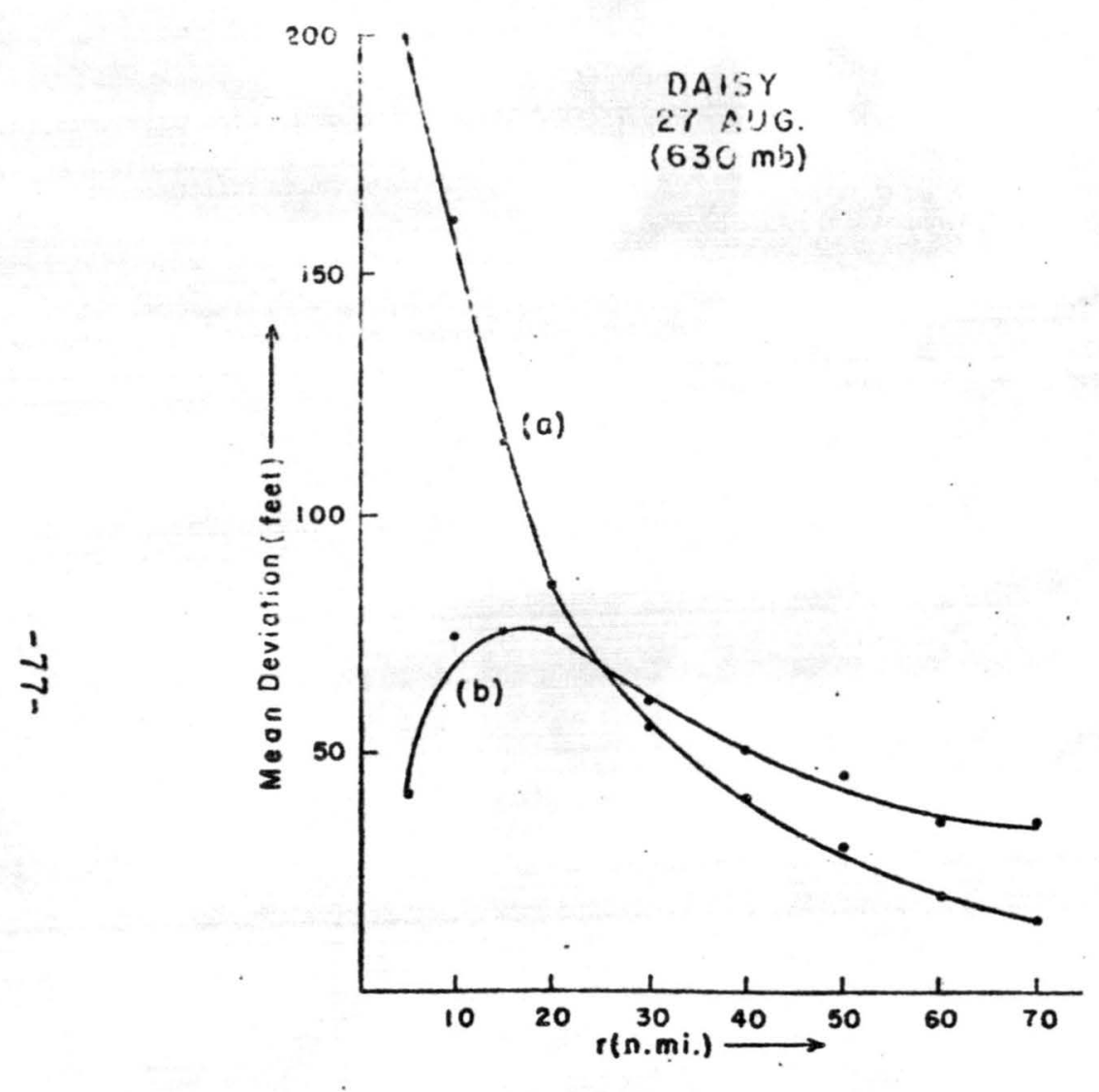

Fig. 65. $630 \mathrm{mb}, 27$ Aug.

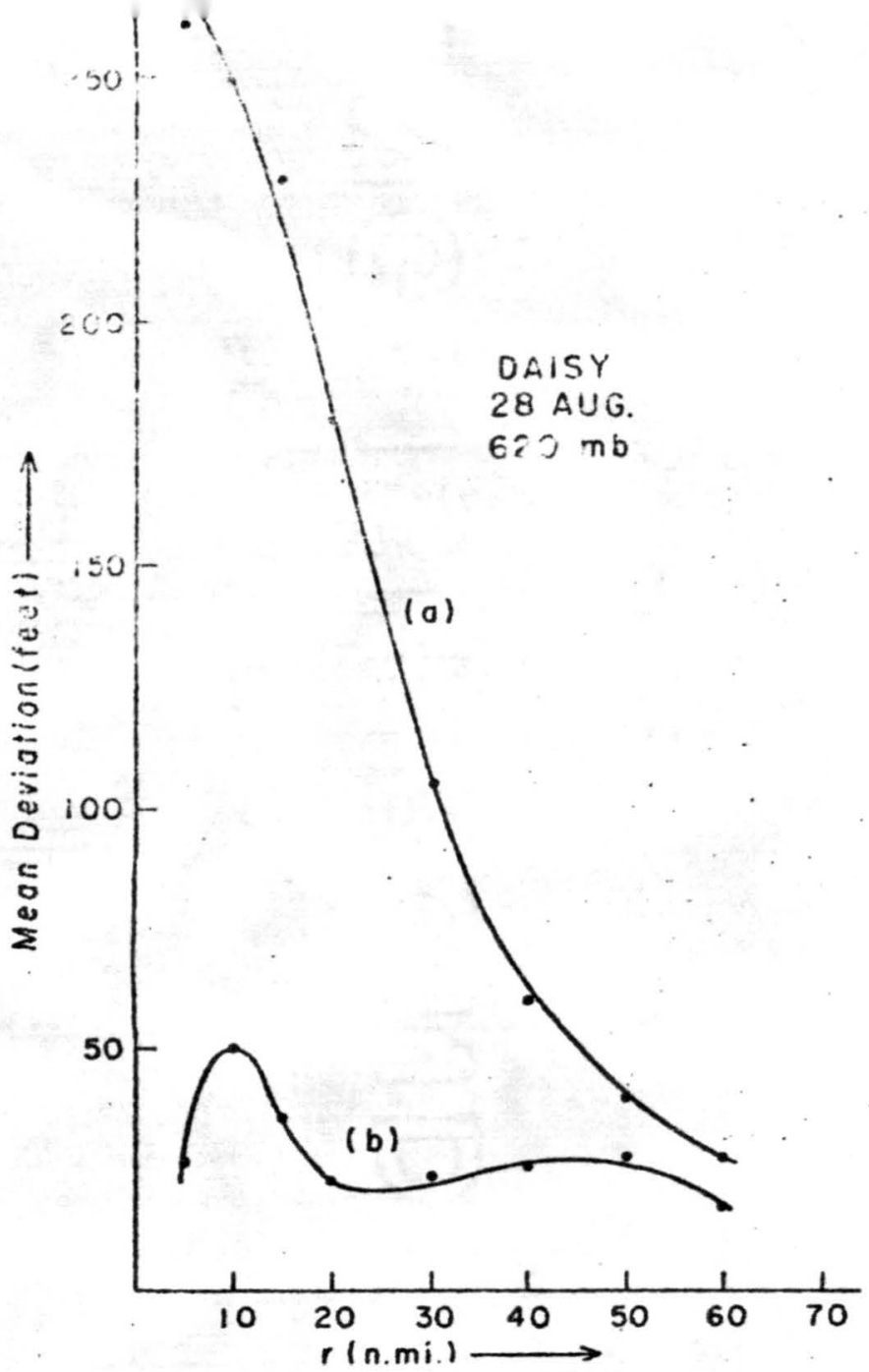

Fig. 66. $620 \mathrm{mb}, 28 \mathrm{Aug}$.

Curve (a) -- Mean deviation of the $D_{g r}$ profiles of the individual radial flight legs at selected radii from their mean value (feet).

Curve (b) -- Mean deviation of the $D_{\text {act }}$ profiles of the individual radial flight legs at selected radii from their mean value (feet). 


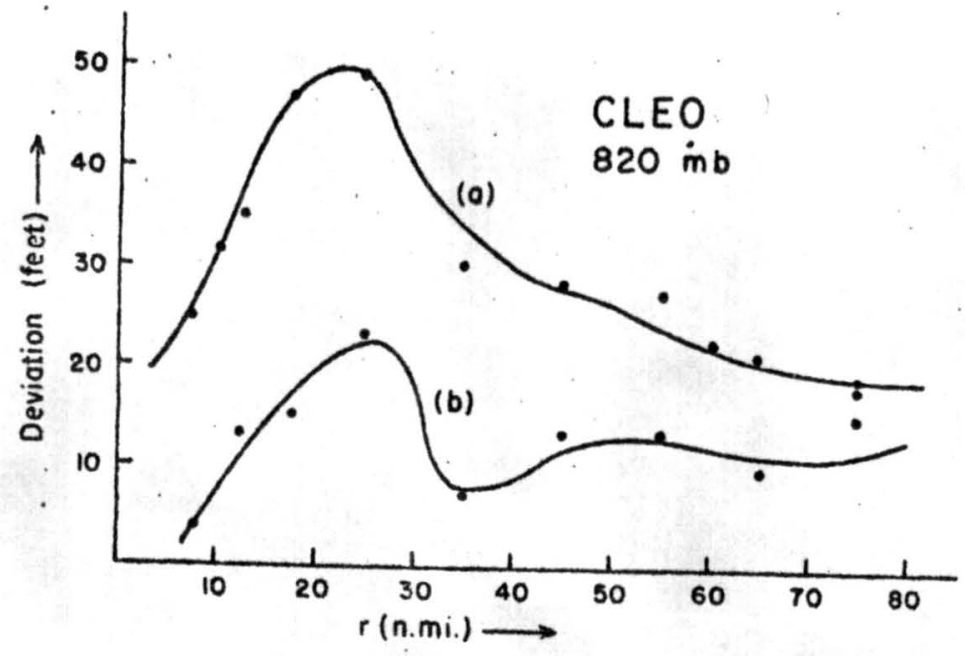

Fig. 67.

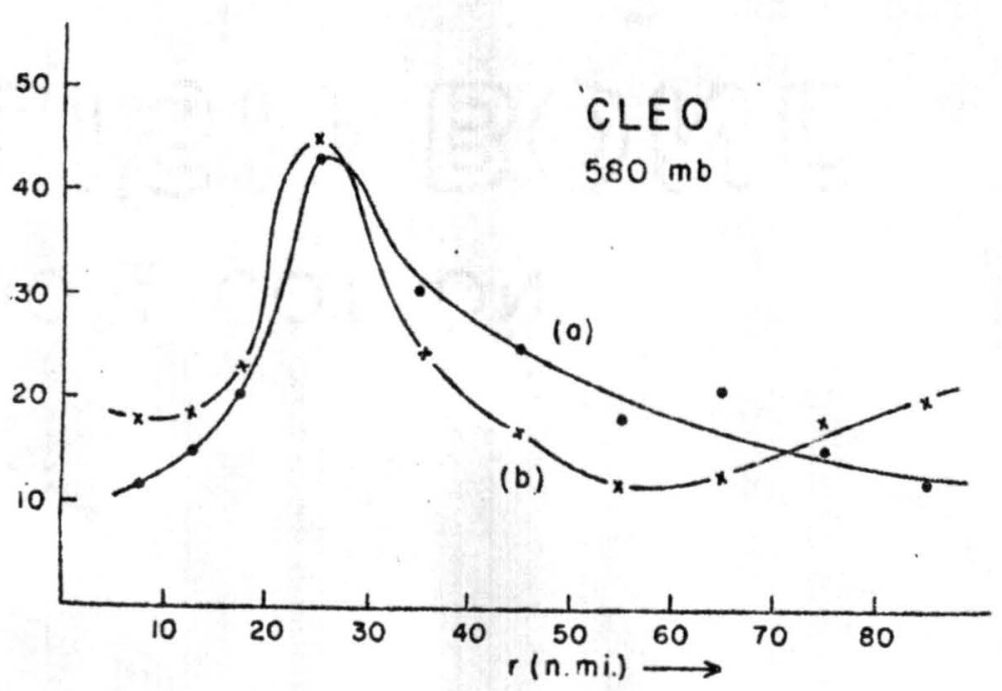

Fig. 68 .

Curve (a) -- Mean deviation of the $D_{g r}$ profiles of the individual radial flight legs at selected radii from their mean value (feet).

Curve (b) -- Mean deviation of the $D_{\text {act }}$ profiles of the individual radial flight legs at selected radii from their mean value (feet). 


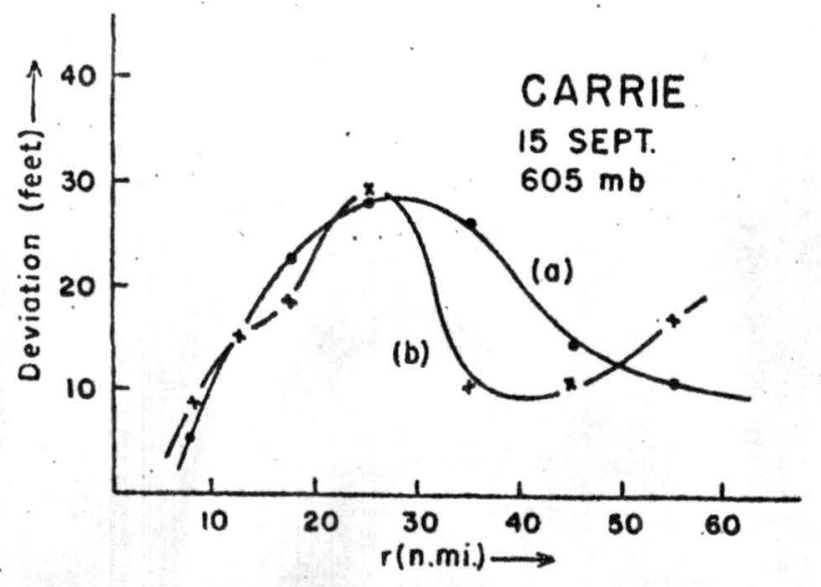

Fig. 69 .

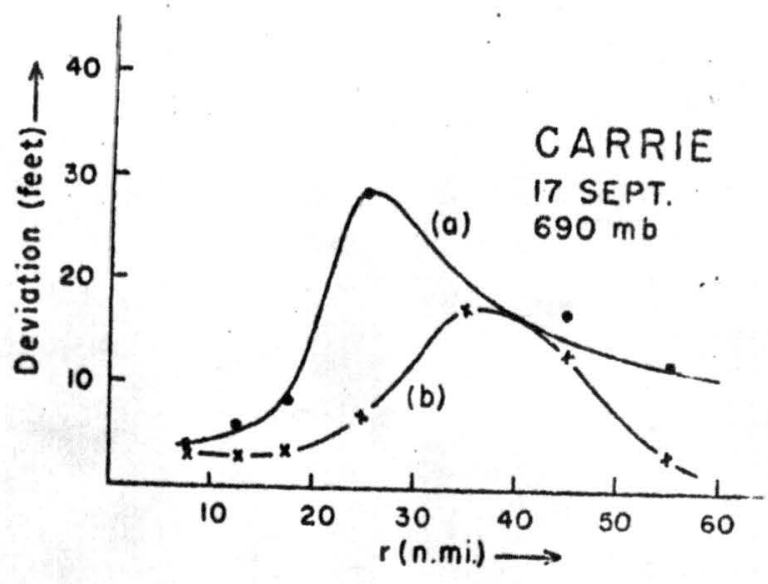

Fig. 70.

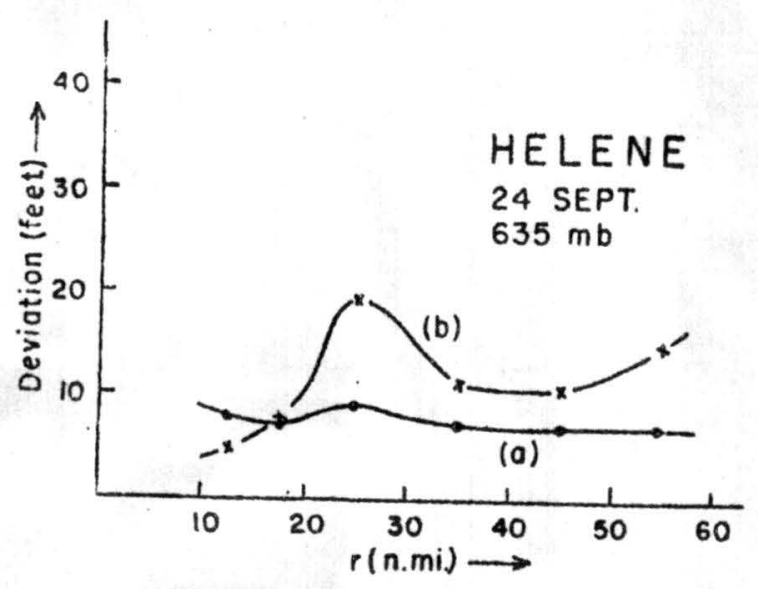

Fig. 71 .

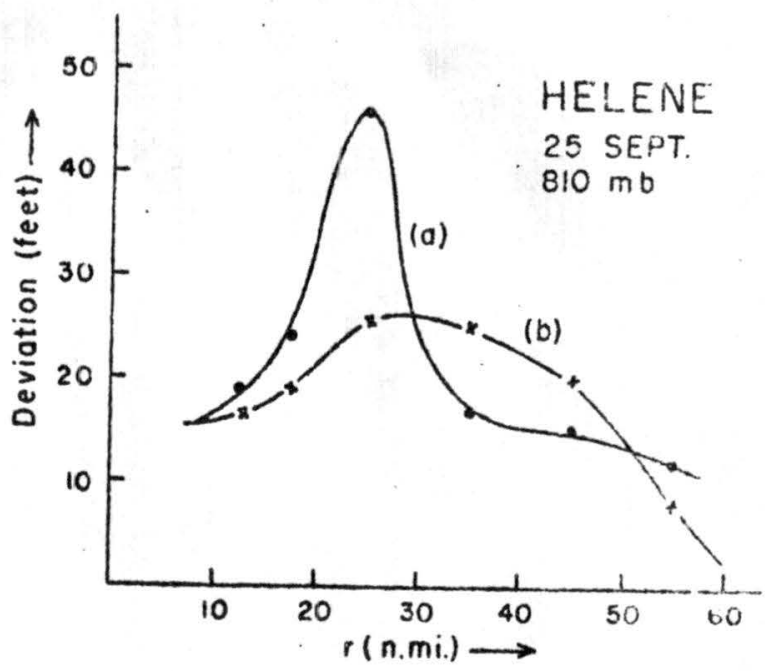

Fig. 72.

Curve (a) -- Mean deviation of the $\mathrm{D}_{\mathrm{gr}}$ profiles of the individual radial flight legs at selected radii from their mean value (feet).

Curve (b) -- Mean deviation of the $D_{\text {act }}$ profiles of the individual radial flight legs at selected radii from their mean value (feet). 


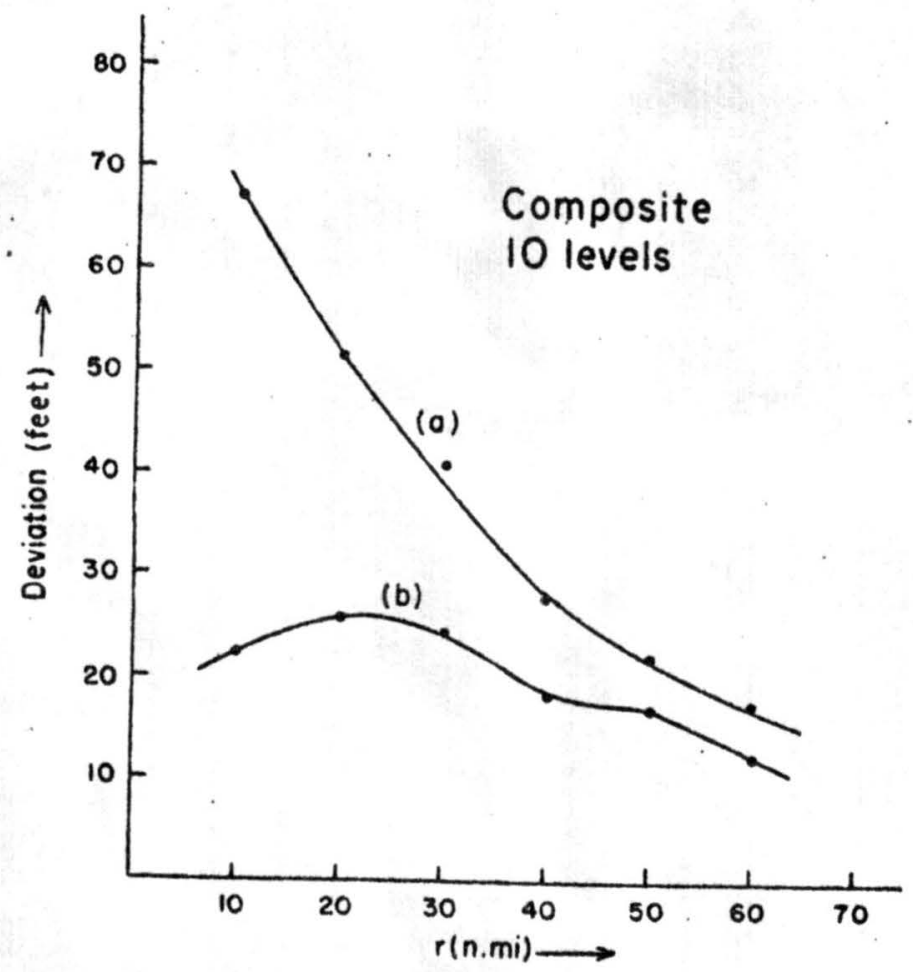

Fig. 73.

Curve (a) -- Mean deviation of the $\mathrm{D}_{\mathrm{gr}}$ profiles of the individual radial flight legs at selected radii from their mean value (feet).

Curve (b) -- Mean deviation of the $D_{\text {act }}$ profiles of the individual radial flight legs at selected radii from their mean value (feet). 


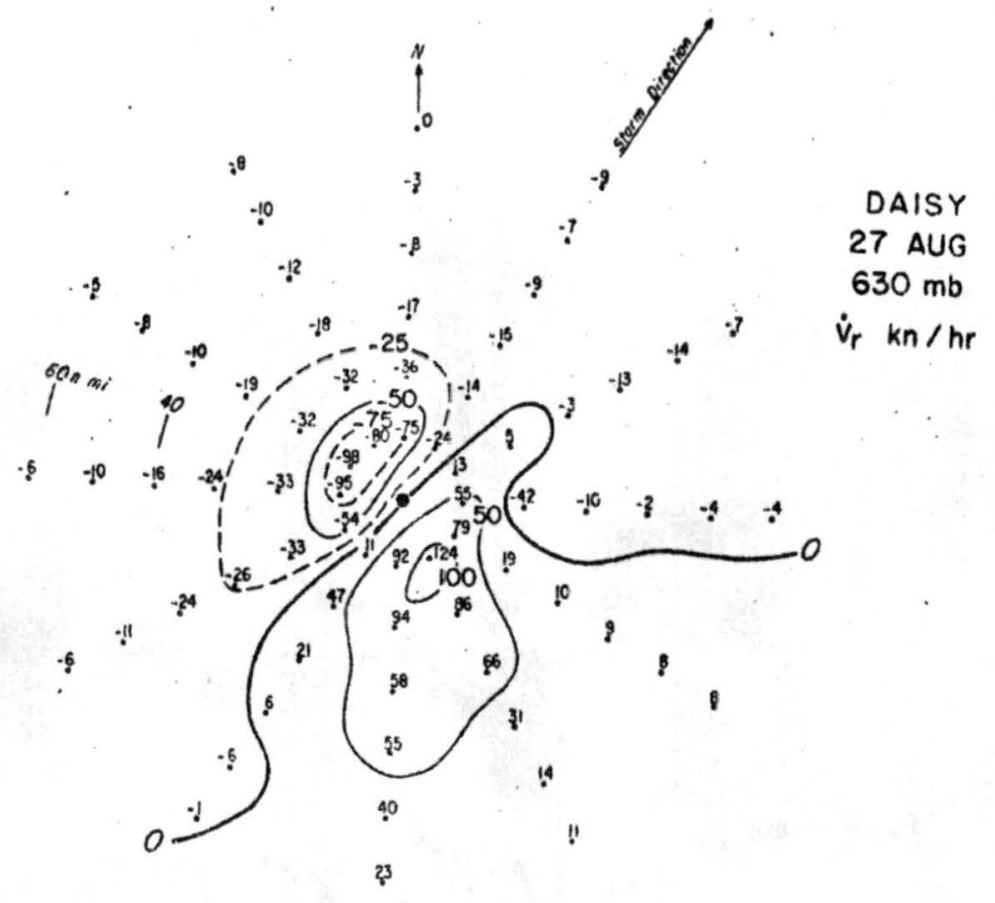

Fig. 74. Field of $\dot{\mathrm{v}}_{\mathrm{r}}$ (knots/hour)

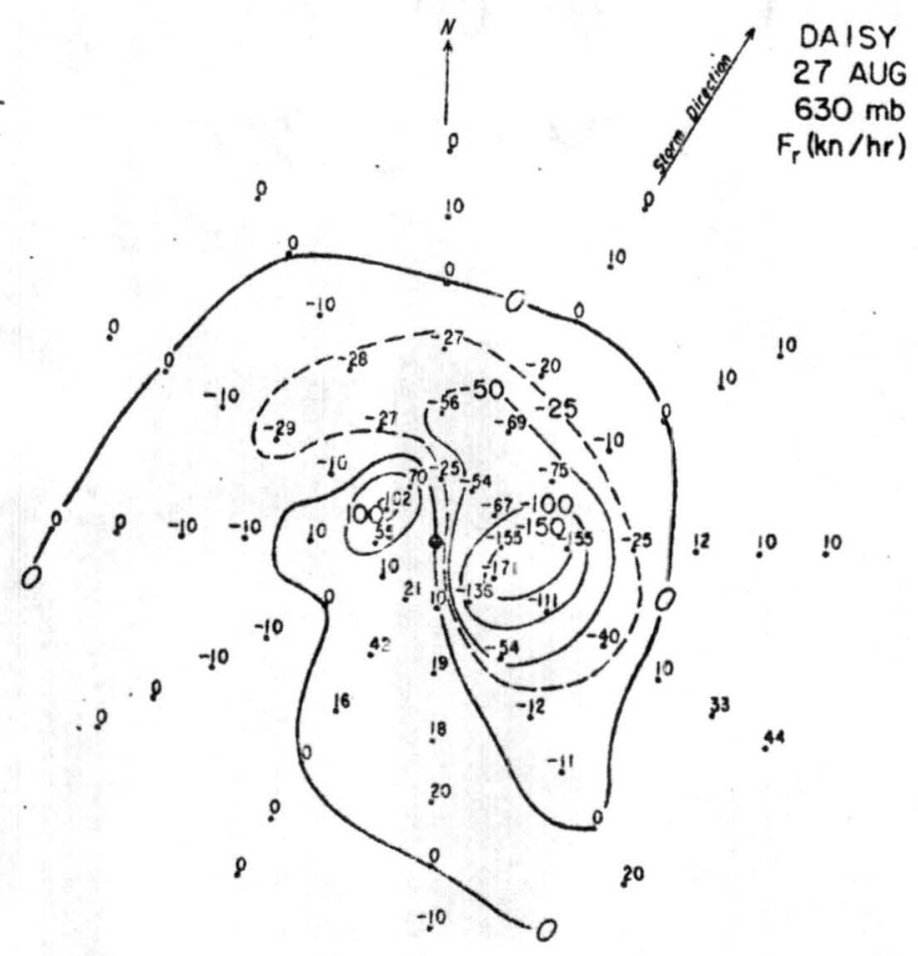

Fig. 75. Computed frictional force (knots/hour) 


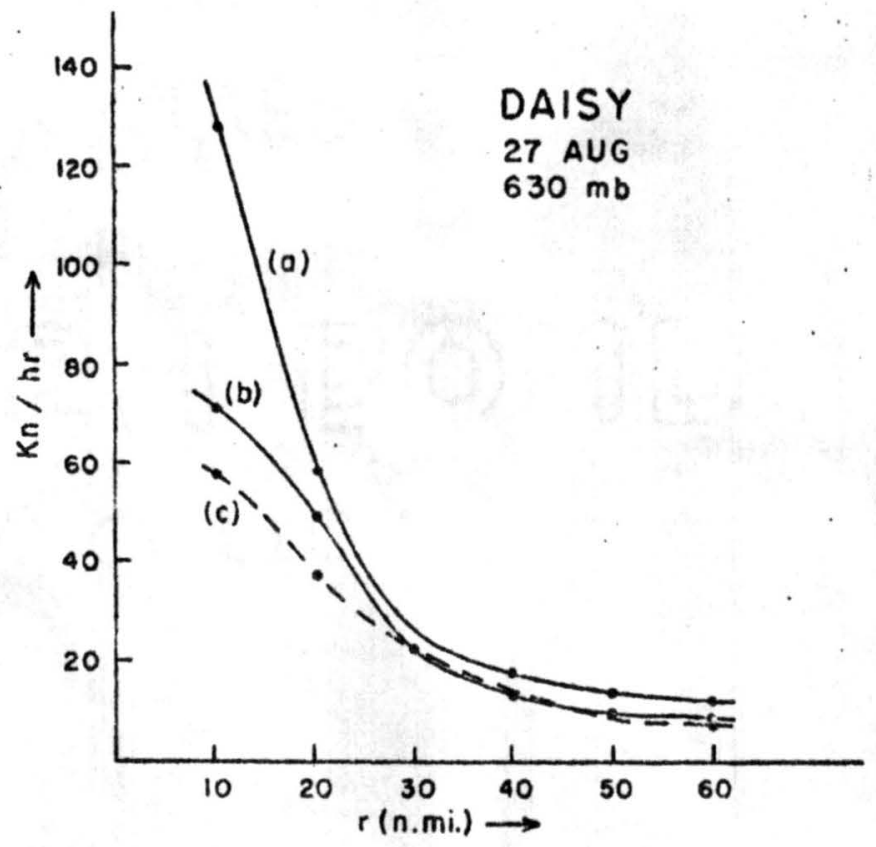

Fig. 76.

Curve (a) - $\left.\dot{\mathrm{v}}_{\mathrm{r}}\right)_{\mathrm{gr}}$ averaged around the storm without respect to sign (units of knots/hour).

Curve (b) $-F_{r}$ averaged around the storm without respect to sign (units of knots/hour).

Curve (c) -- $\dot{v}_{\mathbf{r}}$ averaged around the storm without respect to sign (units of knots/hour). 


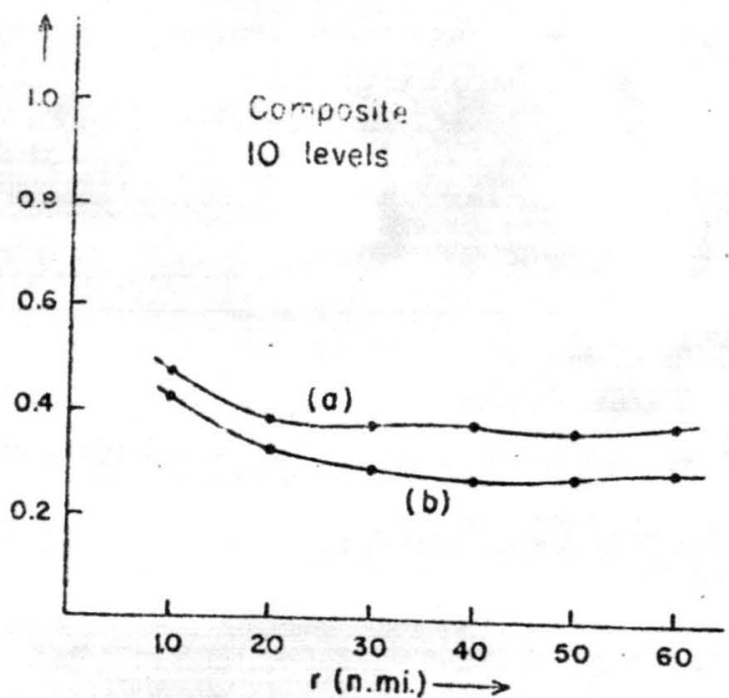

Fig. 77 .

Curve (a) - $\frac{\overline{F_{r}}}{f \bar{v}_{\theta}+\frac{v_{\theta}^{2}}{r}}$ averaged around storm

without respect to sign.

Curve (b) -- $\frac{\overline{F_{r}}}{\mathrm{~g} \frac{\partial D}{\partial r}}$ averaged around storm

without respect to sign.

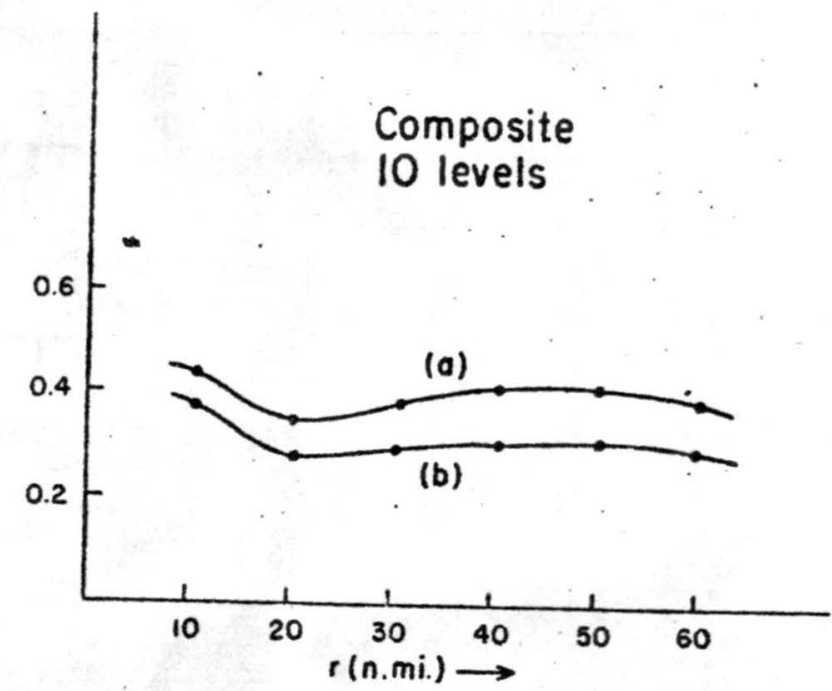

Fig. 78 .

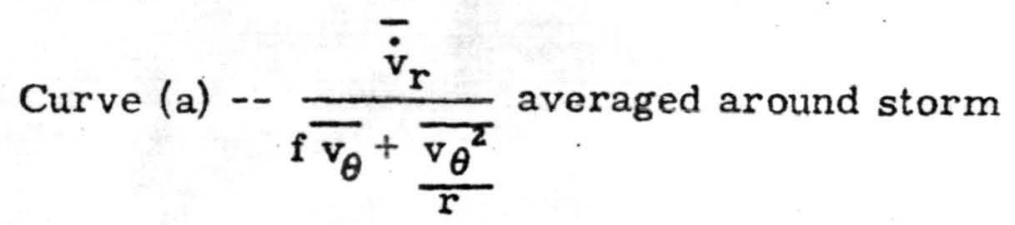

without respect to sign

Curve (b) -- $\frac{\overline{\mathrm{v}}_{r}}{\frac{\partial \mathrm{D}}{\partial \mathbf{r}}}$ averaged around storm without respect to sign. 


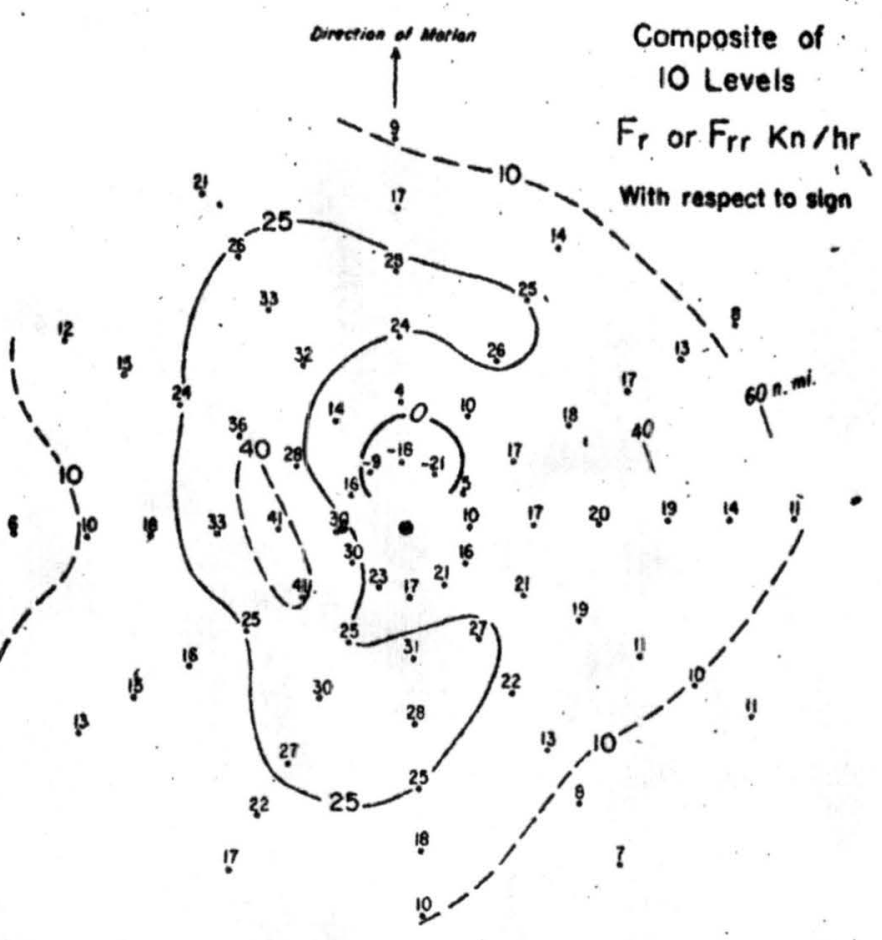

Fig. 79 .

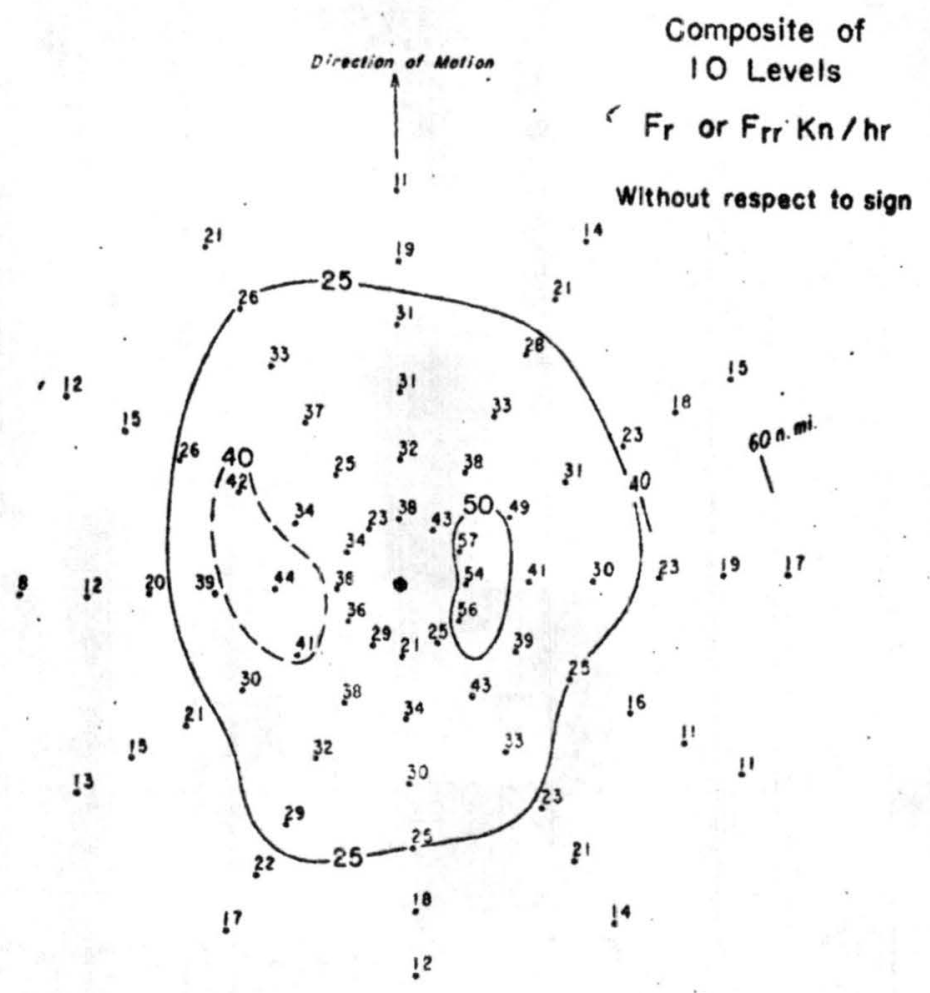

Fig. 80. 


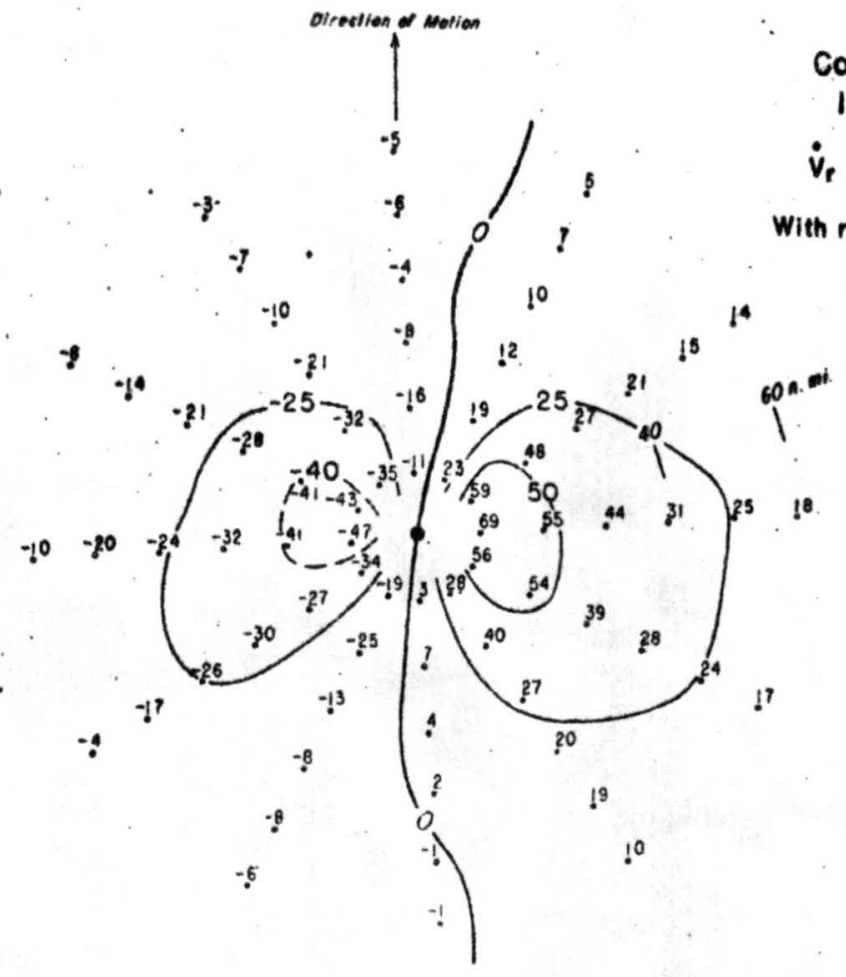

Composite of 10 Lovels

$\mathrm{Kn} / \mathrm{hr}$

With respeet to sign

Fig. 81 .

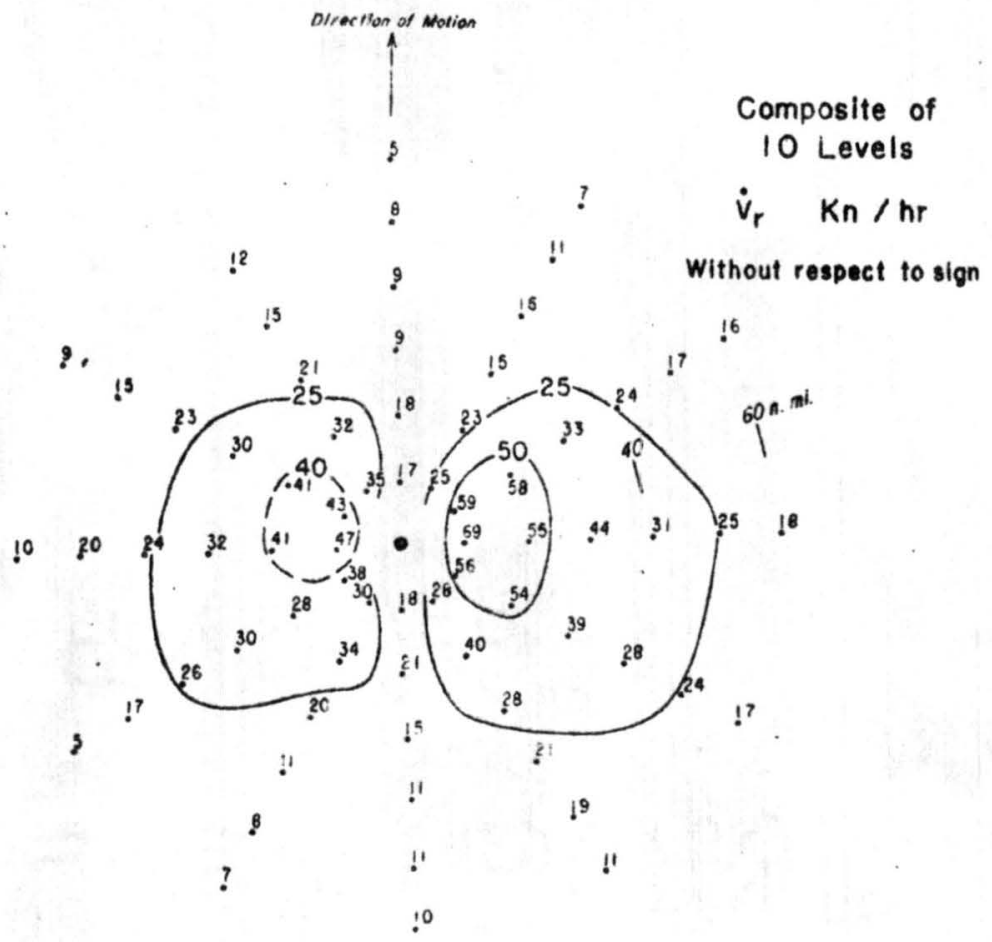

Fig. 82 . 


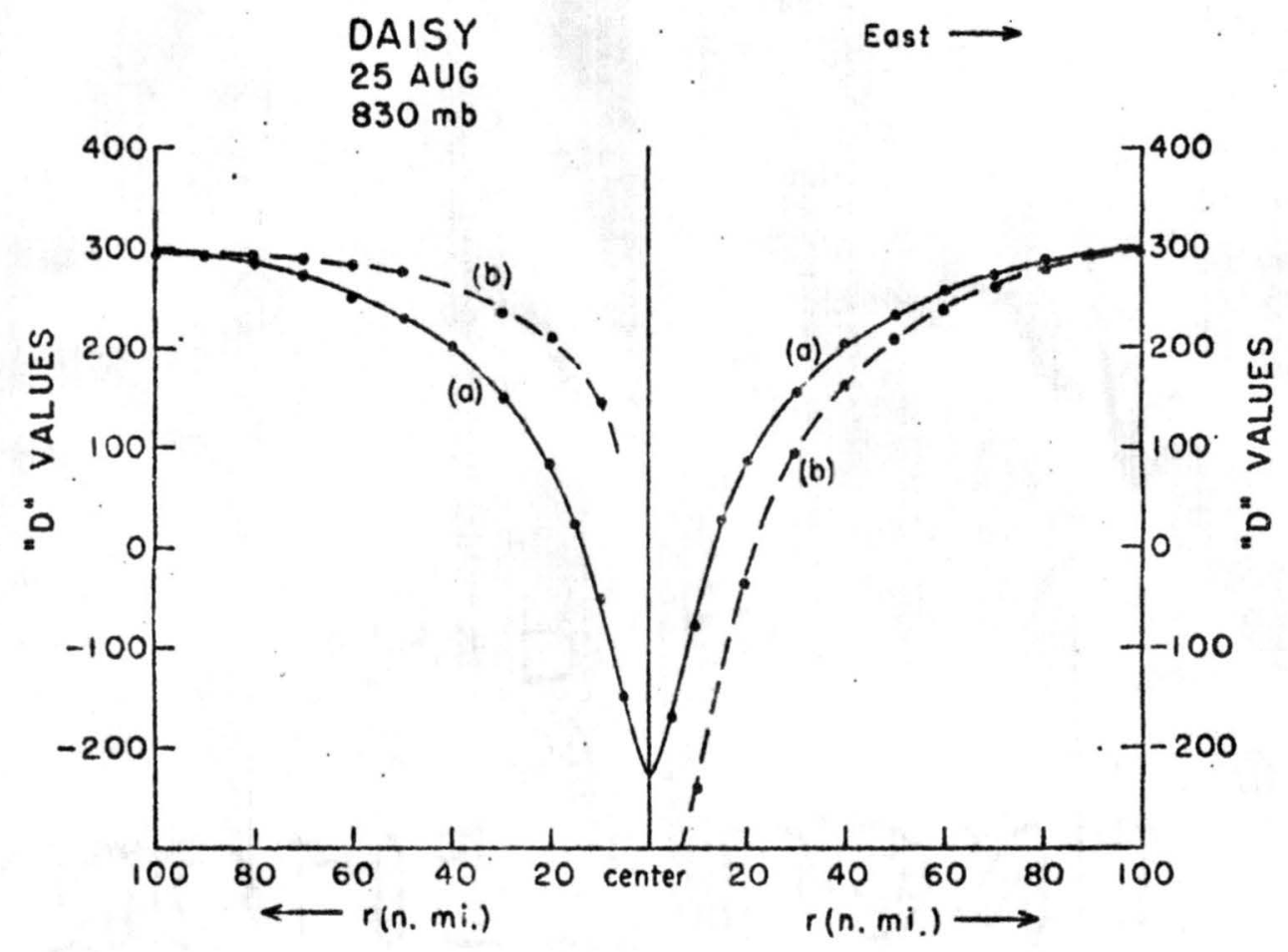

Fig. 83.

Curve (a) -- Observed D-profile ( $\left.D_{\text {act }}\right)$

Curve (b) -- Computed D-profile necessary to balance observed wind pattern $\left(D_{g r}\right)$ 


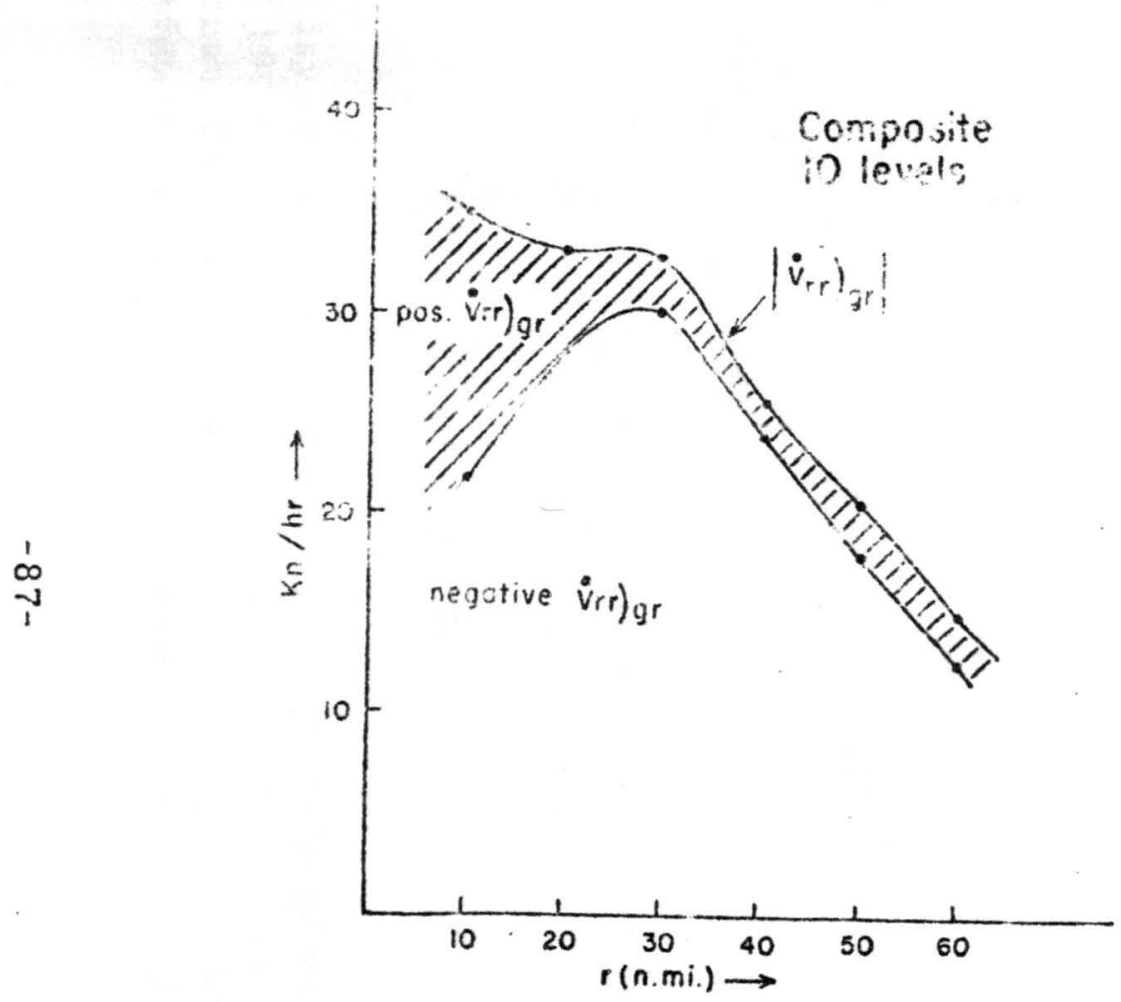

Fig. 84.

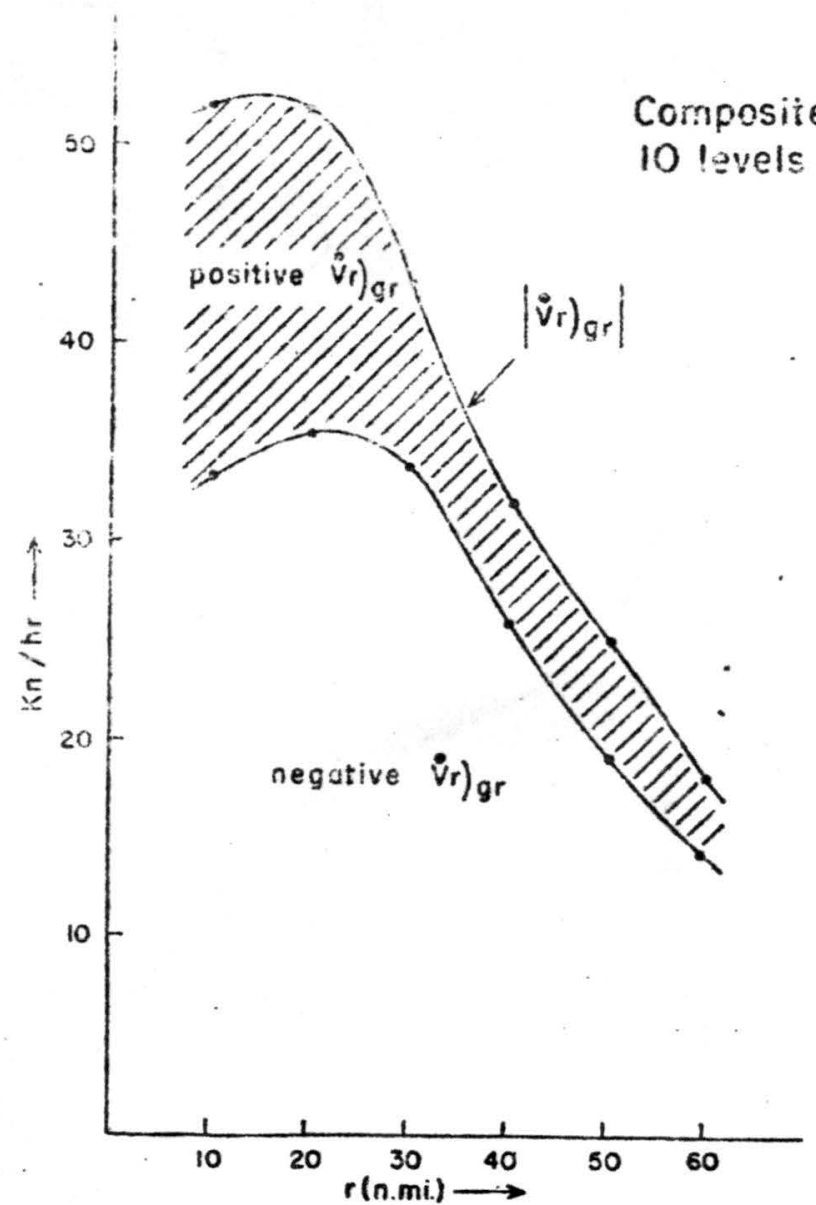

Fig. 85.

$\left|\dot{v}_{r_{g r}^{\prime}}\right|$ and $\left|\dot{v}_{\mathrm{rr}^{\prime}}{ }_{\mathrm{gr}}\right|$ divided into their positive and negative parts after averaging around the storms at selected radii. Ilustrates the predominance of positive $\left(\mathrm{D}_{\mathrm{gr}}-\mathrm{D}_{\mathrm{act}}\right)$ i.e. -- dominance of pressure gradient over centrifugal and Coriolis accelerations, or excess warming. 


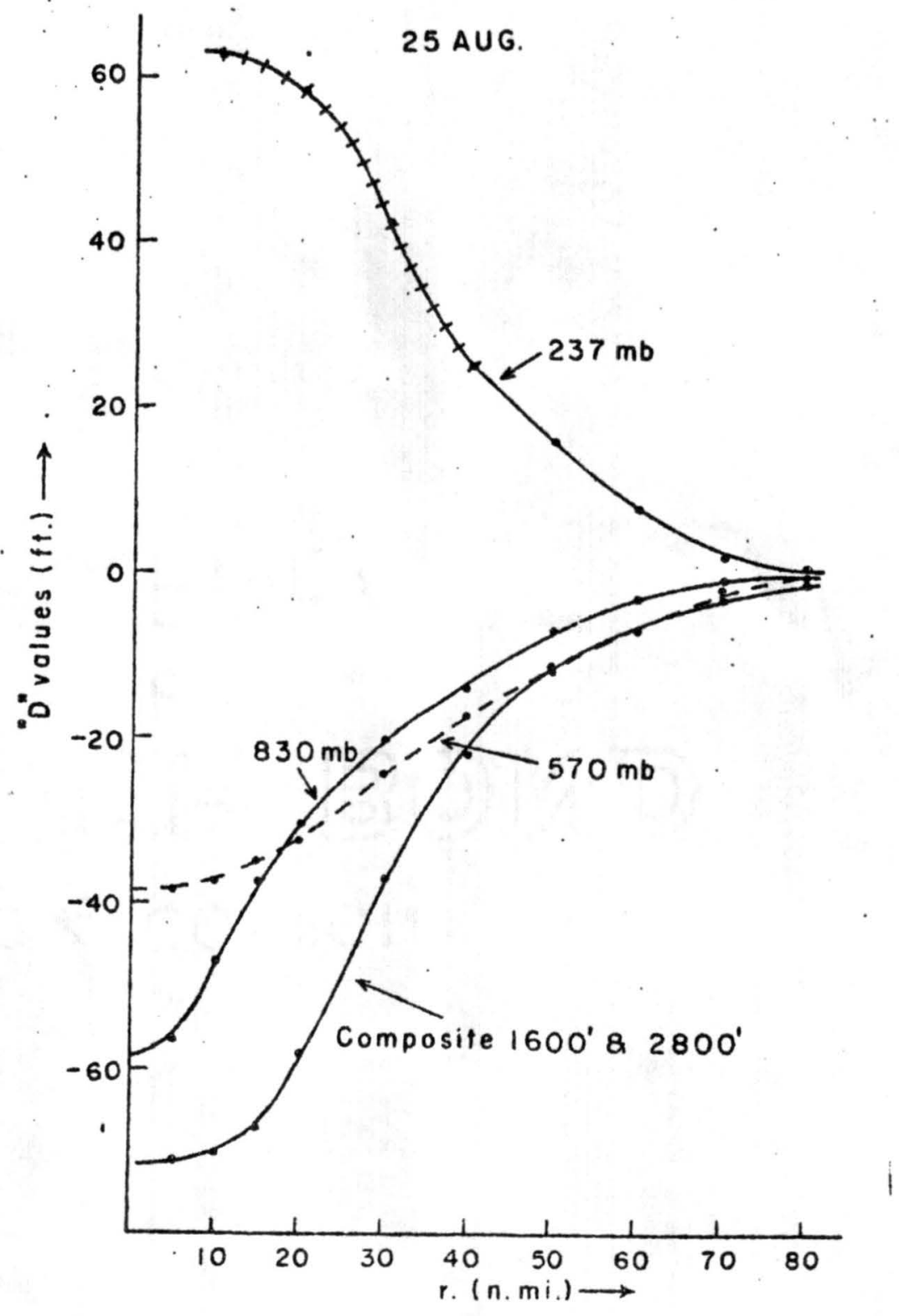

Fig. 86.

Daisy accumulated plot of $\left(D_{\text {act }}-D_{g r}\right)$ from outside reference of $100 \mathrm{n}$. mi. to inside of the storm. Values have been averaged around the storm at selected radii. Negative values of $\left(D_{\text {act }}-D_{\text {gr }}\right)$ indicate stronger pressure gradient than corresponding wind field. 
25 AUG.

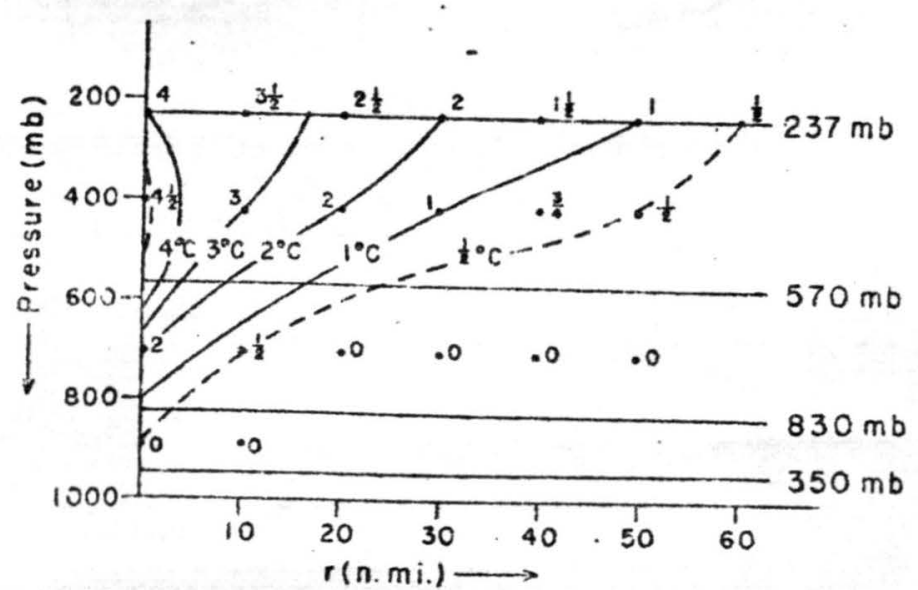

Fig. 87. Vertical cross-section of the computed temperature increase from the temperature values outside the storm area. Values in ${ }^{\circ} \mathrm{C} .25$ Aug.
25 AUG.

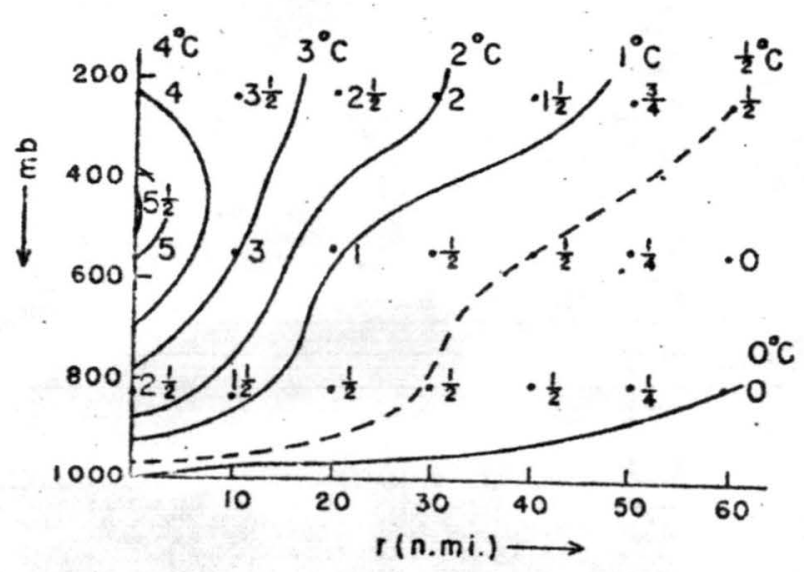

Fig. 88. Vertical cross-section of the observed (aircraft) temperature increase $\left({ }^{\circ} \mathrm{C}\right)$ from the temperature values outsicle of the storm. 25 Aug. 

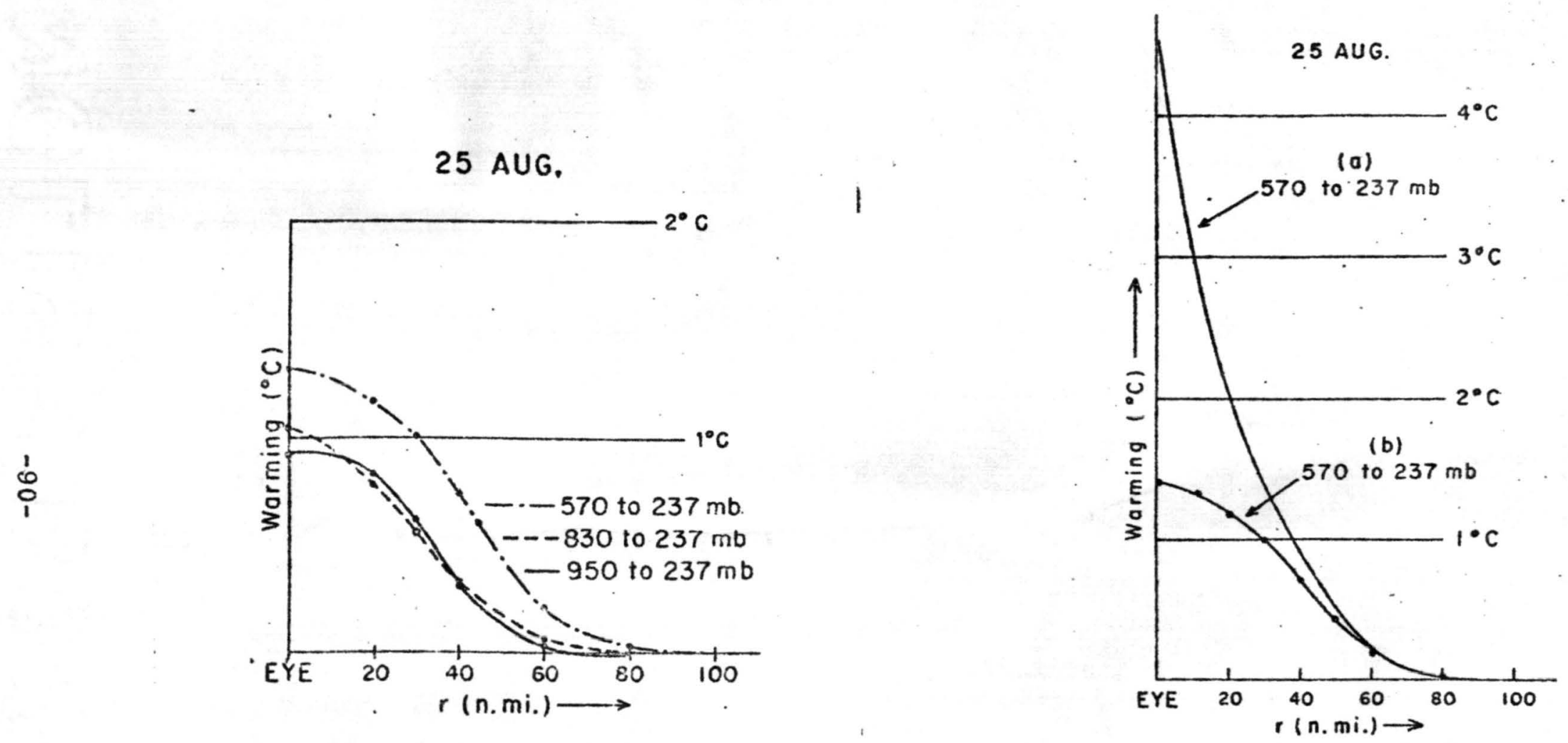

Fig. 89. Excess warming ${ }^{\circ} \mathrm{C}$ between particular pressure layers corresponding to the imbalance of the pressure over the

$\left(f \cdot \bar{v}_{\theta}+\frac{\overline{v_{\theta}^{2}}}{r}\right.$ ) acceleration.

Fig. 90. Comparison of actual warming (curve a) with the acceleration warming (curve b) between 570 and $237 \mathrm{mb}$. Values in ${ }^{\circ} \mathrm{C}$. 25 Aug. 25 Aug. 